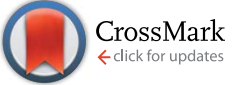

Cite this: RSC Adv., 2017, 7, 1671

Received 4th October 2016 Accepted 28th November 2016

DOI: $10.1039 / c 6 r a 24734 b$

www.rsc.org/advances

\title{
Towards macrocyclic ionic liquids: novel ammonium salts based on tetrasubstituted $p$-tert- butylthiacalix[4]arenes $\uparrow$
}

\author{
Pavel L. Padnya, ${ }^{\text {ab }}$ Elena A. Andreyko, ${ }^{a}$ Polina A. Gorbatova, ${ }^{c}$ Viktor V. Parfenov, ${ }^{c}$ \\ Ildar Kh. Rizvanov ${ }^{d}$ and Ivan I. Stoikov*a
}

\begin{abstract}
Water-insoluble ionic liquids based on $p$-tert-butylthiacalix[4]arenes tetrasubstituted at the lower rim with amide and quaternary ammonium groups containing alkyl, phenyl, ester, phthalimide, glycine, alanine and glycylglycine groups in cone and 1,3-alternate conformations were synthesized. It was established that macrocycles containing quaternary ammonium fragments with alkyl, phenyl and ester groups at the nitrogen atom in cone conformation melt lower by $8-31{ }^{\circ} \mathrm{C}$ than 1,3 -alternate stereoisomers. It was shown that the introduction of the bis(trifluoromethylsulfonyl)imide anion as a counterion in the structure of quaternary ammonium salts based on thiacalix[4]arenes led to a substantial decrease in the melting point of the above salts.
\end{abstract}

\section{Introduction}

Synthesis of ionic liquids for extraction and determination of organic compounds is one of the promising areas of investigation in modern organic chemistry. ${ }^{1-7}$ Water-insoluble solvents have recently been widely used for extraction and separation of biologically significant compounds but they have some technological disadvantages and do not often meet modern environmental standards. The replacement of such solvents is an important applied task. One of the possible solutions to this problem is the use of ionic liquids, e.g., molten salts that are liquids at temperatures below $100{ }^{\circ} \mathrm{C}$. Ionic liquids are mostly non-flammable, synthetically accessible and have negligible vapor pressure; selection of their cations and anions allows adjusting their properties over a wide range. Unique combinations of hydrophobicity and ionic nature, thermal stability and high electrical conductivity of ionic liquids offer new opportunities in the field of organic and analytical chemistry, catalysis and electrochemistry. ${ }^{8-12}$ The development of approaches to creation of new high-performance systems for the extraction and separation of various compounds based on ionic liquids

${ }^{a}$ Kazan Federal University, A.M. Butlerov Chemical Institute, 420008 Kremlevskaya, 18, Kazan, Russian Federation. E-mail: Ivan.Stoikov@mail.ru; Fax: +7-8432-752253; Tel: $+7-8432-337463$

${ }^{b}$ RUDN University, Faculty of Science, Organic Chemistry Department, 117198 Miklukho-Maklaya St., 6, Moscow, Russian Federation

${ }^{c}$ Kazan Federal University, Institute of Physics, 420008, Kremlevskaya, 18, Kazan, Russian Federation

${ }^{d}$ A. E. Arbuzov Institute of Organic and Physical Chemistry, Kazan Scientific Centre of the Russian Academy of Sciences, 420088 Kazan, Russian Federation

$\dagger$ Electronic supplementary information (ESI) available. See DOI: 10.1039/c6ra24734b and functionalized macrocycles, e.g., cyclodextrins, cucurbit $[n]$ urils, (thia)calix $[n]$ arenes, pillar $[n]$ arenes, crown ethers is of great interest. ${ }^{13-17}$

There are two basic approaches to the creation of such systems: (1) synthesis of the macrocyclic compounds soluble in ionic liquids, and (2) design of ionic liquids containing macrocyclic fragment as their cation or anion (Fig. 1).

Despite large synthetic and conformational diversity of (thia) calix[4]arenes as molecular building platform, ${ }^{18-24}$ there are only
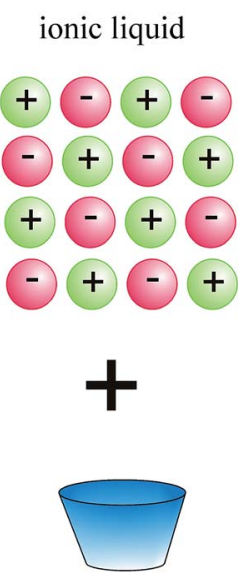

macrocycle
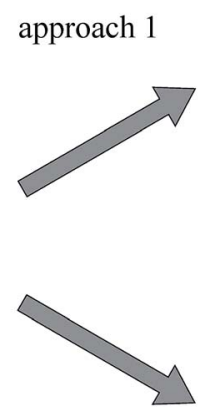

approach 2
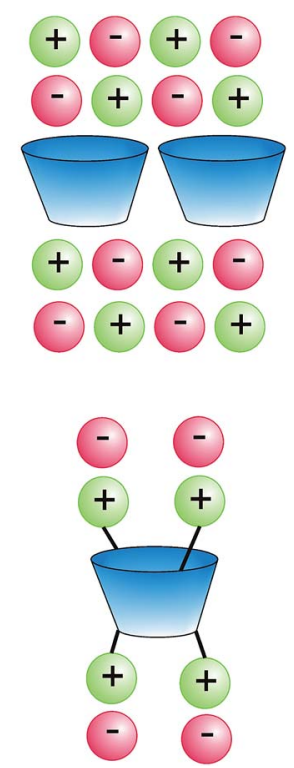

Fig. 1 Two basic approaches to the creation of macrocyclic ionic liquids based on ionic liquids and macrocyclic compounds. 
few examples of the synthesis of ionic liquids based on them. ${ }^{7,25}$ It should be noted that the macrocyclic ionic liquids described in the literature ${ }^{7,25,26}$ belong to the pyridinium and imidazolium derivatives. Synthesis of ionic liquids based on quaternary ammonium salt is one of the most important tasks in modern organic chemistry. ${ }^{27}$ Ease of synthesis, low cost and non-toxicity are the advantages of quaternary ammonium fragments. According to the examples in the literature of chemistry of ionic liquids $^{28-34}$ and (thia)calixarenes, ${ }^{1-7,35}$ we can assume that the introduction of quaternary ammonium fragments at the lower rim of $p$-tert-butylthiacalix[4]arene may be promising for the synthesis of macrocyclic ionic liquids capable to the molecular recognition of target species.

In this work, the synthesis of $p$-tert-butylthiacalix[4]arenes tetrasubstituted at the lower rim with quaternary ammonium groups in cone and 1,3-alternate conformations as potential ionic liquids is described.

\section{Results and discussion}

\section{Synthesis of $p$-tert-butylthiacalix[4]arenes containing quaternary ammonium groups}

To synthesize ionic liquids based on the $p$-tert-butylthiacalix[4] arenes containing quaternary ammonium groups, the reaction of the compounds 1-4 with different alkylating reagents in acetonitrile under reflux has been studied. Alkyl iodides and alkyl bromides were selected as highly reactive alkylating agents. Iodomethane and iodoethane as two simplest homologues were chosen. Based on these compounds, we can verify previously described in the literature suggestion ${ }^{36}$ that the increase in the length of the alkyl substituent led to decrease of the melting point. According to the literature, ${ }^{36}$ it was also assumed that the introduction of planar $\pi$-aromatic ring systems and the ester groups at the lower rim of the macrocycle could result in the synthesis of tetraalkylammonium derivatives of $p$-tert-butylthiacalix[4]arene in cone and 1,3-alternate conformations with low melting points. Thus, benzyl bromide, ethyl bromoacetate and pentyl bromoacetate were used from this consideration. It is interesting to note that in case of iodomethane and iodoethane the reaction was carried out at room temperature because alkyl iodides are more reactive than alkyl bromides.

It was found that the reactivity of the macrocycles $\mathbf{2}$ and $\mathbf{4}$ containing tertiary amino groups with ethyl substituents at the lower rim was lower than the reactivity of the thiacalix[4]arenes 1 and 3 with tertiary amino groups with methyl substituents. Probably, this is due to steric hindrance at the amino nitrogen atom. The increase of reaction time from 8 to 48 hours led to the compounds 5-9, 14-18, 22-26 and 31-35 with $85-98 \%$ yields (Scheme 1). It should be noted that all the synthesized macrocycles 5-9, 14-18, 22-26 and 31-35 are water-soluble. The solubility of the thiacalix[4]arenes 5-9 and 14-18 in cone conformation was much higher than that of the 1,3-alternate stereoisomers 22-26 and 31-35.

To synthesize ionic liquids able to recognize various substrates, stating from low molecular compounds to biomacromolecules, synthesis of the quaternary ammonium salts
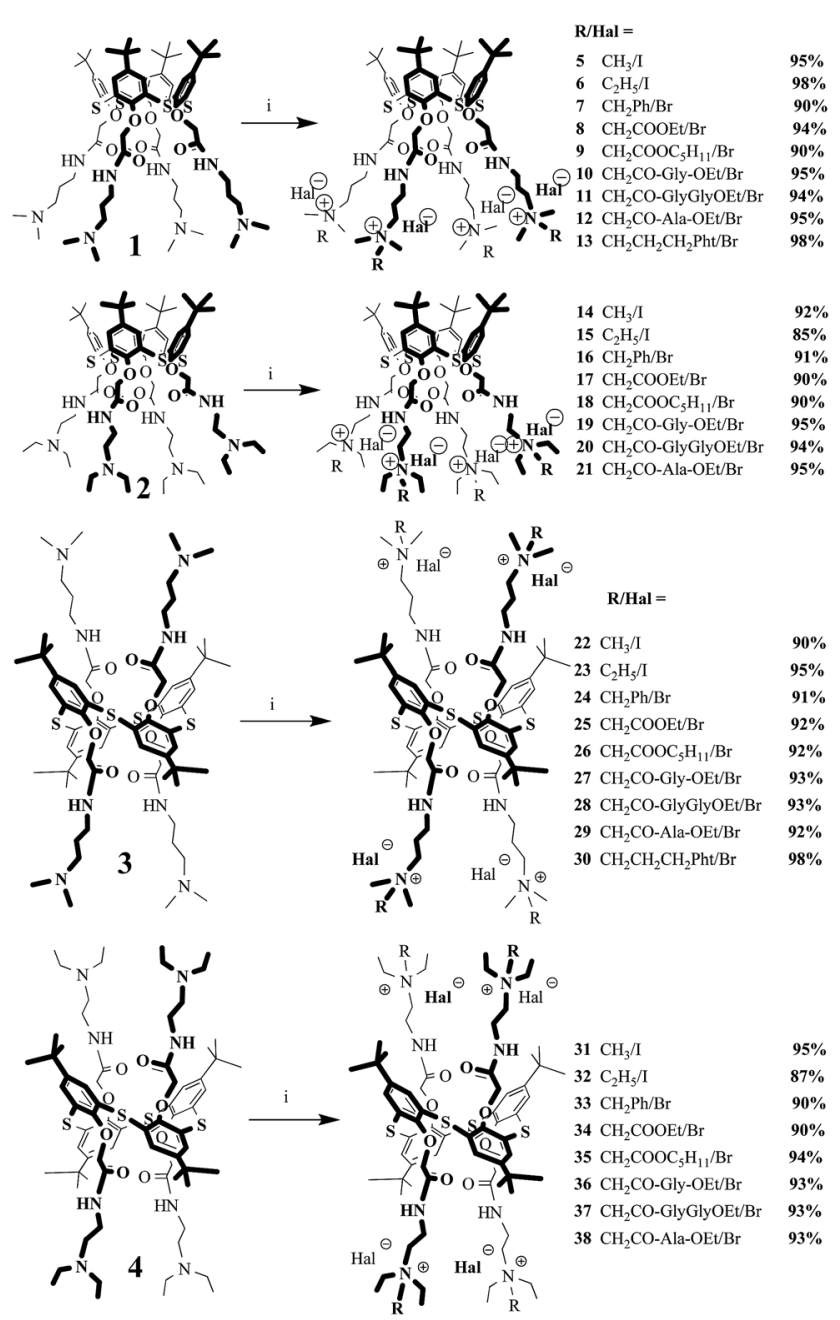

Scheme 1 Reagents and conditions: i - R-Hal, $\mathrm{CH}_{3} \mathrm{CN}$, reflux.

based on $p$-tert-butylthiacalix[4]arenes containing peptide and phthalimide fragments has been performed. $N$-Bromoacetylglycine ethyl ester, $\mathrm{N}$-bromoacetyl-glycylglycine ethyl ester, $\mathrm{N}$ bromoacetyl-L-alanine ethyl ester and $N$-(3-bromopropyl) phthalimide were chosen as alkylating reagents. The presence of $\alpha$-amino acid groups in the structure of $p$-tert-butylthiacalix [4] arenes is necessary for increasing the efficiency of interaction of the macrocycles with biomacromolecules (proteins and DNA) based on the formation of hydrogen bonds. The introduction of phthalimide groups in the structure of macrocycles is also of great interest because the compounds with phthalimide fragments can be used as intercalators for interaction with DNA or for detection of proteins by hydrophobic interactions. ${ }^{37}$

The interaction of ethyl esters of $\mathrm{N}$-bromoacetyl-glycine, $\mathrm{N}$ bromoacetyl-glycylglycine and $N$-bromoacetyl-alanine with aminothiacalix[4]arenes 1-4 containing methyl and ethyl groups at the nitrogen atom in cone and 1,3-alternate conformations was studied earlier. Quaternary ammonium salts 1012, 19-21, 27-29 and 36-38 with amino acid and peptide groups were obtained ${ }^{38}$ (Scheme 1).

It is interesting to note that the reaction of the amines 1-4 with $N$-(3-bromopropyl)phthalimide in acetonitrile under $8 \mathrm{~h}$ 
Table 1 Melting points $\left({ }^{\circ} \mathrm{C}\right.$ ) of the macrocycles 5-21 (cone) and 2238 (1,3-alternate)

\begin{tabular}{|c|c|c|c|c|}
\hline \multirow[b]{2}{*}{$\mathrm{R} / \mathrm{Hal}^{-}$} & \multicolumn{2}{|c|}{$\mathrm{NH}\left(\mathrm{CH}_{2}\right)_{3} \mathrm{~N}^{+}\left(\mathrm{CH}_{3}\right)_{2} \mathrm{R}$} & \multicolumn{2}{|c|}{$\mathrm{NH}\left(\mathrm{CH}_{2}\right)_{2} \mathrm{~N}^{+}\left(\mathrm{C}_{2} \mathrm{H}_{5}\right)_{2} \mathrm{R}$} \\
\hline & Cone & 1,3-Alternate & Cone & 1,3-Alternate \\
\hline $\mathrm{CH}_{3} / \mathrm{I}$ & $192(5)$ & $197(22)$ & $159(14)$ & $156(31)$ \\
\hline $\mathrm{C}_{2} \mathrm{H}_{5} / \mathrm{I}$ & $165(6)$ & $215(23)$ & $163(15)$ & $173(32)$ \\
\hline $\mathrm{CH}_{2} \mathrm{Ph} / \mathrm{Br}$ & $135(7)$ & $150(24)$ & $139(16)$ & $131(33)$ \\
\hline $\mathrm{CH}_{2} \mathrm{COOC}_{2} \mathrm{H}_{5} / \mathrm{Br}$ & $112(8)$ & $123(25)$ & $118(17)$ & $115(34)$ \\
\hline $\mathrm{CH}_{2} \mathrm{COOC}_{5} \mathrm{H}_{11} / \mathrm{Br}$ & $106(9)$ & $103(26)$ & $135(\mathbf{1 8})$ & $88(35)$ \\
\hline $\mathrm{CH}_{2}$ CO-Gly-OEt $/ \mathrm{Br}$ & $114(10)$ & $112(27)$ & $111(19)$ & $114(36)$ \\
\hline $\mathrm{CH}_{2} \mathrm{CO}-$ GlyGlyOEt/Br & $113(11)$ & $120(28)$ & $120(20)$ & $124(37)$ \\
\hline $\mathrm{CH}_{2} \mathrm{CO}-\mathrm{Ala}-\mathrm{OEt} / \mathrm{Br}$ & $116(12)$ & $118(29)$ & $116(21)$ & $118(38)$ \\
\hline $\mathrm{CH}_{2} \mathrm{CH}_{2} \mathrm{CH}_{2} \mathrm{Pht} / \mathrm{Br}$ & $152(13)$ & $154(30)$ & - & - \\
\hline
\end{tabular}

reflux resulted in formation of the products only in the case of the compounds 1 and 3 containing tertiary amino groups with $N, N$-dimethyl substituents at the lower rim in cone and 1,3alternate conformations. The compounds $\mathbf{1 3}$ and $\mathbf{3 0}$ were synthesized with excellent yields ${ }^{38}$ (Scheme 1). According to the ${ }^{1} \mathrm{H}$ NMR spectroscopy, the mixture of differently substituted products difficult for separation was obtained for the macrocycles $\mathbf{2}$ and $\mathbf{4}$ containing tertiary amine groups with $N, N$-diethyl substituents at the lower rim. The increase of the reaction time up to 40 hours did not lead to the formation of the target products. Probably, reactivity of the macrocycles $\mathbf{2}$ and $\mathbf{4}$ is reduced by steric hindrance in tertiary amino groups with $\mathrm{N}, \mathrm{N}$ diethyl substituents against that of the compounds $\mathbf{1}$ and $\mathbf{3}$ containing tertiary amino groups with methyl substituents.

Melting point is one of main characteristics of ionic liquids (see Table 1 for the synthesized macrocycles 5-21 (cone) and 2238 (1,3-alternate)). Conformation changes and length of the alkyl substituents led to a slight decrease in the melting point is agreement with the literature. ${ }^{36}$ Introduction of benzyl and ester groups in the structure of macrocycles decreased the melting points by $30-40{ }^{\circ} \mathrm{C}$, in contrast to $5-7{ }^{\circ} \mathrm{C}$ predicted in the literature. ${ }^{36}$ However, main goal has not been achieved. All the obtained salts 5-21 (cone) and 22-38 (1,3-alternate) melt above $100{ }^{\circ} \mathrm{C}$ except the macrocycle 35 containing pentyl acetate fragment.

\section{Synthesis of ionic liquids based on $p$-tert-butylthiacalix[4] arenes containing alkyl, ester, aromatic, peptide and phthalimide fragments}

As shown in the literature ${ }^{39}$ replacement of the halide ions by bis(trifluoromethylsulfonyl)imide ions considerably decreased melting point. This can be explained by the fact that the increase in the size of anions decreased symmetry of the molecule obtained $^{39}$ (Fig. 2). Thus, the study of the interaction of the compounds 5-38 with lithium bis(trifluoromethylsulfonyl)imide in water at room temperature was next step of the work (Scheme 2, Fig. 2).

The structure and composition of the synthesized compounds 39-72 were determined by the ${ }^{1} \mathrm{H}$ and ${ }^{13} \mathrm{C}$ NMR, IR spectroscopy, mass spectrometry and elemental analysis. The ${ }^{13} \mathrm{C}$ NMR spectrum of the compounds $39-72$ exhibits a quartet

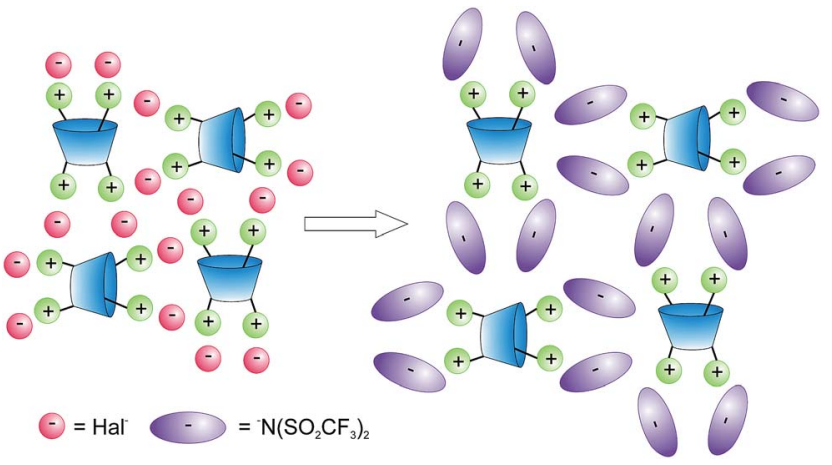

Fig. 2 Possible scheme of the changes of crystal packing of the $p$ tert-butylthiacalix[4]arene ammonium salts at the replacement of halide ions by bis(trifluoromethylsulfonyl)imide ions.

at $120 \mathrm{ppm}$; these signals correspond to anion ${ }^{-} \mathrm{N}\left(\mathrm{SO}_{2} \mathrm{CF}_{3}\right)_{2}$ (Fig. S67-S100, ESI $\dagger$ ).

The configuration of $p$-tert-butylthiacalix[4]arenes can be studied by two-dimensional NMR spectroscopy. However, the configuration of the compounds 5-72 can be also determined by one-dimensional ${ }^{1} \mathrm{H}$ NMR spectroscopy based on specific proton signals.

The conformational differentiation of the cone and 1,3alternate stereoisomers of the $p$-tert-butylthiacalix[4]arenes tetrasubstituted at the lower rim can be recognized by chemical shifts of the tert-butyl group, aromatic ring, oxymethylene and amide protons in the ${ }^{1} \mathrm{H}$ NMR spectra (Table S1, ESI $\dagger$ ). In the compounds 22-38 and 56-72 in the 1,3-alternate conformation, the protons of the $-\mathrm{OCH}_{2}-$ and amide groups are located in the shielded zone of neighboring aromatic rings of the macrocycle, and their signals in the ${ }^{1} \mathrm{H}$ NMR spectrum are recorded at stronger fields (3.96-4.20 and 8.01-8.38 ppm, respectively) than those of the macrocycles 5-21 and 39-55 in cone conformation (4.79-5.03 and 8.23-9.13 ppm, respectively). The chemical shifts of the aromatic protons depend less on the conformation of the macrocyclic cavity. They drift by only $0.19-0.25 \mathrm{ppm}$ upfield from cone 5-21 and 39-55 (7.30-7.45 ppm) to 1,3-alternate 2238 and 56-72 (7.59-7.61 ppm) stereoisomers. This is an evidence of the shielding effect of neighboring aryl fragments in the cone stereoisomer on the aryl protons of macrocycle ring. The protons of the tert-butyl groups of the cone stereoisomers 5-21 and 39-55 were found at a stronger field (1.07-1.12 ppm) against corresponding proton signals of the 1,3-alternate stereoisomers 22-38 and 56-72 (1.19-1.22 ppm). This effect is probably due to the spatial location of the tert-butyl groups of the 1,3-alternate stereoisomer shielded by neighboring fragments of the macrocycle.

It should be noted that the proton signals in the ${ }^{1} \mathrm{H}$ NMR spectra of the quaternary ammonium salts 5-38 (Table S1, Fig. S1-S16, ESI $\dagger$ ) containing halide anions, and the proton signals of initial salts 39-72 (Table S1, Fig. S17-S50, ESI $\dagger$ ) containing bis(trifluoromethylsulfonyl)imide anions have identical multiplicity and exert very similar chemical shifts. It can be explained that these compounds are able to form solvent-separated ion pairs in solution. 


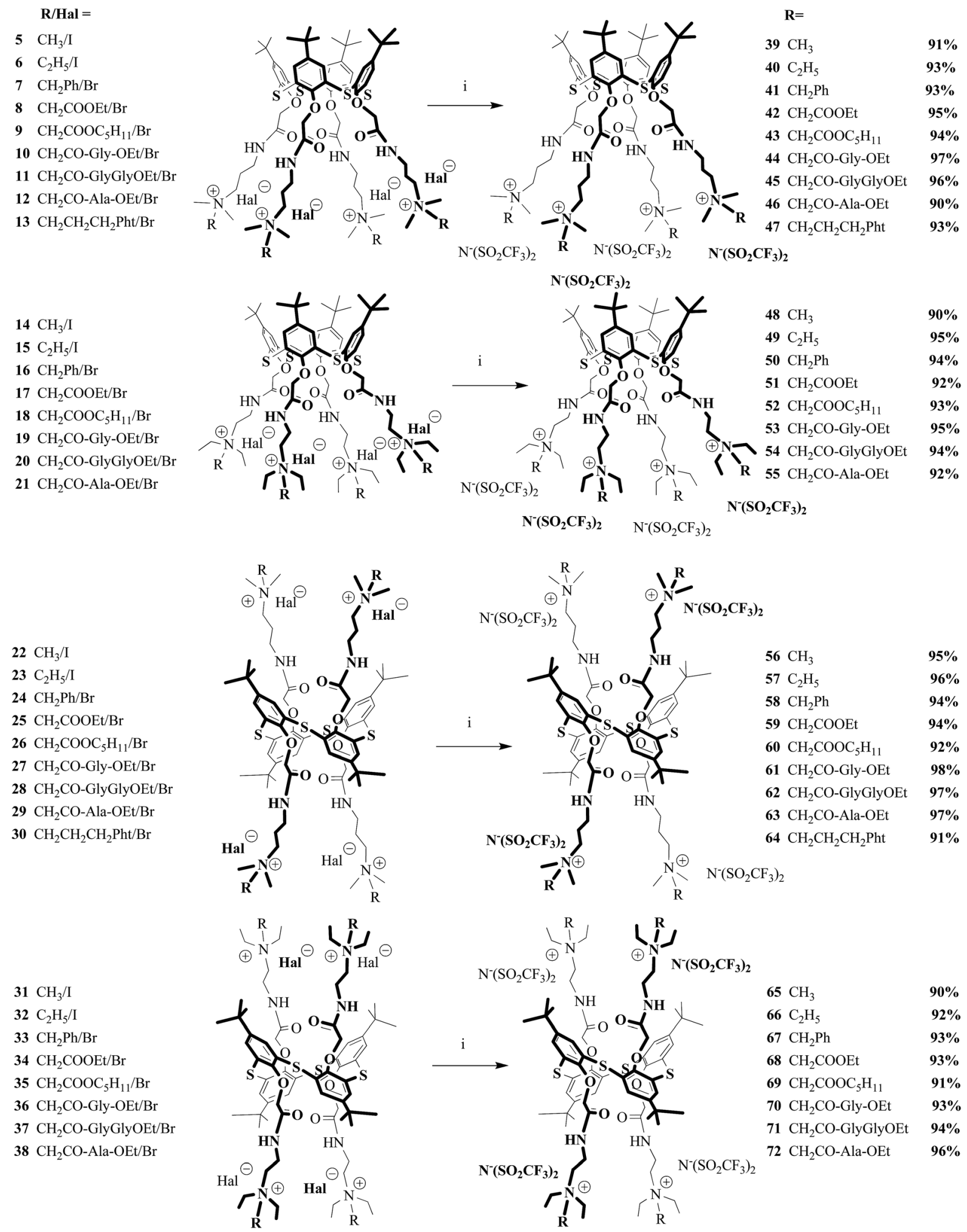

Scheme 2 Reagents and conditions: $\mathrm{i}-\mathrm{Li}^{+} \mathrm{N}^{-}\left(\mathrm{SO}_{2} \mathrm{CF}_{3}\right)_{2}, \mathrm{H}_{2} \mathrm{O}$.

Melting points of the synthesized thiacalix[4] arenes 39-72 are presented in Table 2. One can see (Tables 1 and 2) that the replacement of halide ions by bis(trifluoromethylsulfonyl)imide ions leads to significant decrease in the melting points of the thiacalix[4]arenes studied. All the synthesized macrocycles
39-72 containing bis(trifluoromethylsulfonyl)imide anions melt below $100{ }^{\circ} \mathrm{C}$, except the product 56 (Table 2).

One can see (Table 2) that stereoisomerism of the macrocycles 39-43, 48-52, 56-60, 65-69 has an impact on their melting points. In the case of the cone (39-43, 48-52) 
Table 2 Melting points $\left({ }^{\circ} \mathrm{C}\right)$ of the macrocycles 39-55 (cone) and 56-72 (1,3-alternate)

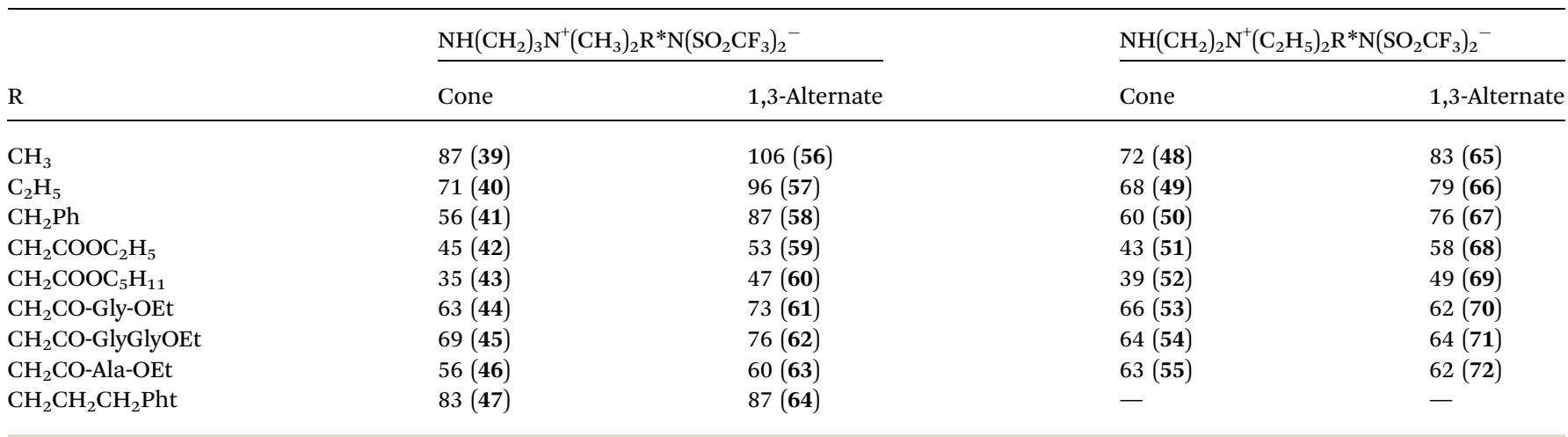

stereoisomers, their melting points are lower by $8-31^{\circ} \mathrm{C}$ against those of the thiacalix[4]arenes $(\mathbf{5 6 - 6 0}, \mathbf{6 5 - 6 9})$ in 1,3-alternate conformation. It is well known that packing density of the molecules in the crystal lattice is a major factor affecting the melting point of the substance. More symmetrical molecules have denser packing in crystal and higher melting point. Obviously, molecular symmetry of the cone (39-43, 48-52) stereoisomers results in maximal spatial separation of the bulk lipophilic tert-butyl and charged ammonium groups and hence in decrease of the packing density and appropriate reduction of their melting point. On the other hand, in the case of the symmetric 1,3-alternate (56-60, 65-69) stereoisomers that show higher melting points, alternation of tert-butyl and ammonium groups at adjacent aryl fragments led to denser packing of the molecules. However, the introduction of additional amide groups with amino acid residues (Gly, Ala) in the structure of thiacalix[4]arene compared to the macrocycles 39-43, 48-52, 56-60, 65-69 decreased influence of the macrocycle configuration on their melting points. Obviously, peptide groups able to form hydrogen bonds contribute to the formation of denser packing of the molecules in the crystal. It can be assumed that two opposite factors influence melting points of the thiacalix[4] arenes depending on the structure of macrocycles, i.e., conformation (melting points of the stereoisomers decrease in the range: 1,3-alternate, cone) and presence of the proton-donating $(-\mathrm{NH})$ and proton-accepting (carbonyl) groups (melting points are increased due to the formation of associates). This results in the fact that melting points of the macrocycles $(\mathbf{4 4 - 4 7 , 5 3 - 5 5}$, 61-64, 70-72) in cone and 1,3-alternate conformations are slightly different.

Increasing length of the alkyl substituent of the macrocycle by one $\mathrm{CH}_{2}$ - group leads to decrease of the melting point by $8^{-}$ $9{ }^{\circ} \mathrm{C}$ in good agreement with the literature. ${ }^{36}$ It should also be noted that the compound $\mathbf{4 3}$ containing pentoxy carbonylmethylene groups at the lower rim in cone conformation has the lowest melting point $\left(35^{\circ} \mathrm{C}\right)$. This corresponds closely to the hypothesis about the influence of the ester groups on the melting points of the target products.

Thermal stability and ionic conductivity have been established for the compound $\mathbf{4 3}$ with the lowest melting point. Thermal stability of the compound $\mathbf{4 3}$ toward pyrolysis was investigated by thermogravimetric analysis (Fig. S201, ESI†).
The $5 \mathrm{wt} \%$ loss temperature $\left(T_{5}\right)$ of the compound 43 under nitrogen was equal to $293.5{ }^{\circ} \mathrm{C}$ indicating its high thermal stability. It is known that macrocyclic ionic liquids have lower ionic conductivity in comparison with their non-macrocyclic analogues. ${ }^{26}$ The ionic conductivity of the compound $\mathbf{4 3}$ was evaluated by ac impedance spectroscopy. The ionic conductivity of compound 43 in the bulk state at $324 \mathrm{~K}$ was found to be 6.00 $\times 10^{-7} \mathrm{~S} \times \mathrm{cm}^{-1}$ (Fig. 3) corresponded to moderate ionic conductivity. Fig. 3 shows low- $(120 \mathrm{~Hz})$ and high-frequency $(500$ $\mathrm{kHz}$ ) electrical conductivity exhibiting exponential increase with the temperature. The activation energy for the highfrequency conductivity $E_{\mathrm{A} 1}=0.69 \mathrm{eV}$ is approx. twofold less than that of the low-frequency conductivity $\left(E_{\mathrm{A} 2}=1.24 \mathrm{eV}\right)$. This behavior of the activation energy was also observed for other organic semiconductors ${ }^{42}$ and is usually associated with the processes of the hopping of charge carriers in an inhomogeneous conducting medium. This model supposes the current in organic semiconductors generated by hopping carriers between polyconjugated areas from one to another limited by dielectric barrier created by disordered (non-conjugated) structure. Small activation energy values are typical for the occurrence of carriers within interface area and manifest themselves in the measurements at a high frequency, while measuring at the DC and low frequencies give substantially higher values of the activation energy associated probably with the above barrier hopping between coupling fragments.

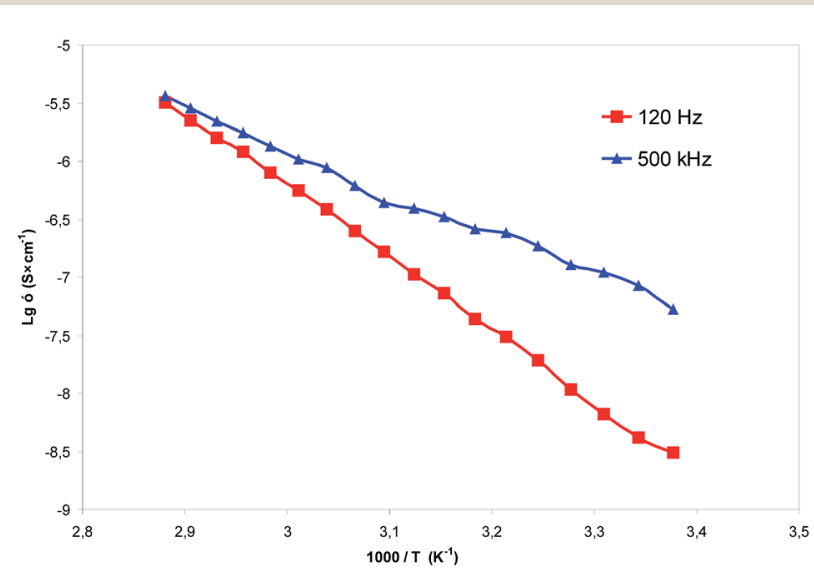

Fig. 3 Ionic conductivity of the thiacalix[4]arene cone 43. 


\section{Conclusions}

Thus, water-soluble and water-insoluble $p$-tert-butylthiacalix[4] arenes tetrasubstituted at the lower rim with amide and quaternary ammonium groups with alkyl, ester, amino acid, peptide and phthalimide fragments in cone and 1,3-alternate conformation were synthesized. The structure and composition of the synthesized macrocycles were determined by the physical methods, i.e., the ${ }^{1} \mathrm{H}$ and ${ }^{13} \mathrm{C}$ NMR, IR spectroscopy, MALDI TOF and ESI mass spectrometry and elemental analysis. It was found that the replacement of halide ions in the synthesized macrocycles by bis(trifluoromethylsulfonyl)imide ions led to water-insoluble salts with melting points below $100{ }^{\circ} \mathrm{C}$. It was shown that the macrocycles containing quaternary ammonium fragments with alkyl, phenyl and ester groups at the nitrogen atom in cone conformation melt lower by $8-31{ }^{\circ} \mathrm{C}$ than $1,3-$ alternate stereoisomers. Macrocyclic water-insoluble ionic liquids synthesized in this work showed high thermal stability and moderate ionic conductivity. These salts can be used in the sensor assemblies for the molecular recognition of the target substrates, e.g., biomacromolecules and cations of heavy and transition metals.

\section{Experimental}

\section{General}

The ${ }^{1} \mathrm{H}$ and ${ }^{13} \mathrm{C}$ NMR spectra of compounds (3-5\% solution in $\left.\mathrm{CDCl}_{3},\left(\mathrm{CD}_{3}\right)_{2} \mathrm{SO}\right)$ were recorded on $400 \mathrm{MHz}$ and $100 \mathrm{MHz}$ Bruker Avance 400 spectrometer using $\mathrm{CDCl}_{3}$ and $\left(\mathrm{CD}_{3}\right)_{2} \mathrm{SO}$ as internal standard.

The IR spectra were recorded on Spectrum 400 (Perkin Elmer) IR spectrometer. The IR spectra from 4000 to $400 \mathrm{~cm}^{-1}$ were considered in this analysis. The spectra were measured with $4 \mathrm{~cm}^{-1}$ resolution and 14 scans co-addition.

Elemental analysis was performed on Perkin-Elmer 2400 Series II instruments.

Mass spectra (MALDI-TOF) were recorded on Ultraflex III mass spectrometer in the 4-nitroaniline matrix.

Mass spectra (ESI) were recorded on an AmaZonX mass spectrometer (Bruker Daltonik GmbH, Germany). The drying gas was nitrogen at $300{ }^{\circ} \mathrm{C}$. The capillary voltage was $4.5 \mathrm{kV}$. The samples were dissolved in acetonitrile (concentration $\sim 10^{-6} \mathrm{~g} \mathrm{ml}^{-1}$ ).

Melting points were determined using Boetius Block apparatus. The purity of the compounds was monitored by melting, boiling points, ${ }^{1} \mathrm{H}$ NMR and thin layer chromatography (TLC) on $200 \mu \mathrm{m}$ UV 254 silica gel plate using UV-light (254 nm).

Conductivity measurements were performed on the RLCmeter E7-20 in "sandwich" type cell at frequencies of $120 \mathrm{~Hz}$ and $500 \mathrm{kHz}$ at temperatures range from room temperature to $74{ }^{\circ} \mathrm{C}$. The sample size was $1 \mathrm{~cm}^{2}$, thickness $-4 \mathrm{~mm}$.

In this work, the following reagents and solvents were used: acetonitrile (chemical pure), benzyl bromide (chemical pure), lithium bis(trifluoromethansulfonyl)imide (Acros Organic), $\mathrm{N}$-(3-bromopropyl)phthalimide (Acros Organic), distilled water, iodomethane (Acros Organic), iodoethane (Acros Organic), ethyl bromoacetate (Acros Organic), pentyl bromoacetate (chemical pure).
$\mathrm{N}$-Bromoacetyl-glycine ethyl ester, $\mathrm{N}$-bromoacetyl-glycylglycine ethyl ester, $N$-bromoacetyl-L-alanine ethyl ester were synthesized according to the literature procedure. ${ }^{38}$

5,11,17,23-Tetra-tert-butyl-25,26,27,28-tetrakis[ $N$ - $\left(3^{\prime}, 3^{\prime}\right.$-dimethylaminopropyl)carbomoylmethoxy]-2,8,14,20-tetrathiacalix[4] arene (cone 1) was synthesized according to the literature procedure. $^{40}$

5,11,17,23-Tetra-tert-butyl-25,26,27,28-tetrakis[ $N$ - $\left(2^{\prime}, 2^{\prime}\right.$-diethylaminoethyl)carbomoylmethoxy]-2,8,14,20-tetrathiacalix[4]arene (cone 2) was synthesized according to the literature procedure. ${ }^{\mathbf{4 0}}$

$5,11,17,23$-Tetra-tert-butyl-25,26,27,28-tetrakis[ $N$-( $\left(3^{\prime}, 3^{\prime}\right.$-dimethylaminopropyl)carbomoylmethoxy]-2,8,14,20-tetrathiacalix[4] arene (1,3-alternate 3$)$ was synthesized according to the literature procedure. ${ }^{38}$

5,11,17,23-Tetra-tert-butyl-25,26,27,28-tetrakis[ $N$-(2', $2^{\prime}$-diethylaminoethyl)carbomoylmethoxy]-2,8,14,20-tetrathiacalix[4]arene (1,3-alternate 4) was synthesized according to the literature procedure. $^{38}$

$5,11,17,23$-Tetra-tert-butyl-25,26,27,28-tetrakis $\left[\left(N-\left(3^{\prime}, 3^{\prime}, 3^{\prime}-\right.\right.\right.$ trimethyl)-ammoniumpropyl)carbomoylmethoxy]-2,8,14,20tetrathiacalix[4]arene tetraiodide (cone 5) was synthesized according to the literature procedure. ${ }^{40}$

$5,11,17,23$-Tetra-tert-butyl-25,26,27,28-tetrakis $\left[\left(N\right.\right.$ - $\left(3^{\prime} 3^{\prime}\right.$-dimethyl-3'-ethyl)ammoniumpropyl)carbomoylmethoxy]-2,8,14,20tetrathiacalix[4]arene tetraiodide (cone 6) was synthesized according to the literature procedure. ${ }^{41}$

$5,11,17,23$-Tetra-tert-butyl-25,26,27,28-tetrakis[ $\left(N-\left(3^{\prime}, 3^{\prime}\right.\right.$-dimethyl-3'-benzyl)ammoniumpropyl)carbomoylmethoxy]-2,8,14,20tetrathiacalix[4]arene tetrabromide (cone 7) was synthesized according to the literature procedure. ${ }^{40}$

$5,11,17,23$-Tetra-tert-butyl-25,26,27,28-tetrakis[ $N$ - $\left(3^{\prime}, 3^{\prime}\right.$-dimethyl-3'-(ethoxycarbonylmethyl)ammoniumpropyl)carbomoylmethoxy]-2,8,14,20-tetrathiacalix[4]arene tetrabromide (cone 8) was synthesized according to the literature procedure. ${ }^{\mathbf{4 1}}$

$5,11,17,23$-Tetra-tert-butyl-25,26,27,28-tetrakis $\left[N\right.$ - $\left(3^{\prime}, 3^{\prime}\right.$-dimethyl- $3^{\prime}-\{($ ethoxycarbonylmethyl)amidocarbonylmethyl $\}$ ammoniumpropyl)carbomoylmethoxy]-2,8,14,20-tetrathiacalix[4]arene tetrabromide (cone 10) was synthesized according to the literature procedure. ${ }^{38}$

$5,11,17,23$-Tetra-tert-butyl-25,26,27,28-tetrakis[ $N$ - $\left(3^{\prime}, 3^{\prime}\right.$-dimethyl- $3^{\prime}-\{([$ ethoxycarbonylmethyl $]$ amidocarbonylmethyl)amidocarbonylmethyl\}ammoniumpropyl)carbomoylmethoxy]-2,8,14,20tetrathiacalix[4] arene tetrabromide (cone 11) was synthesized according to the literature procedure. ${ }^{38}$

$5,11,17,23$-Tetra-tert-butyl-25,26,27,28-tetrakis[ $N$ - $\left(3^{\prime}, 3^{\prime}\right.$-dimethyl-3'-\{(ethoxycarbonyl[S-methyl]methyl)amidocarbonylmethyl $\}$ ammoniumpropyl)carbomoylmethoxy]-2,8,14,20-tetrathiacalix [4] arene tetrabromide (cone 12) was synthesized according to the literature procedure. ${ }^{38}$

5,11,17,23-Tetra-tert-butyl-25,26,27,28-tetrakis[ $N$-( $3^{\prime}, 3^{\prime}$-dimethyl$3^{\prime}-\left\{3^{\prime \prime}\right.$-propylphthalimide $\}$ ammoniumpropyl)carbomoylmethoxy]2,8,14,20-tetrathiacalix[4] arene tetrabromide (cone 13) was synthesized according to the literature procedure. ${ }^{\mathbf{4 1}}$

$5,11,17,23$-Tetra-tert-butyl-25,26,27,28-tetrakis[( $N$-( $2^{\prime}$-methyl$2^{\prime}, 2^{\prime}$-diethyl)ammoniumethyl)carbomoylmethoxy]-2,8,14,20tetrathiacalix[4]arene tetraiodide (cone 14) was synthesized according to the literature procedure. ${ }^{40}$ 
5,11,17,23-Tetra-tert-butyl-25,26,27,28-tetrakis $\left[\left(\mathrm{N}\right.\right.$ - $\left(2^{\prime}, \mathrm{2}^{\prime}\right.$-diethyl-2' $-\{($ ethoxycarbonylmethyl)amidocarbonylmethyl $\}$ ammoniumethyl)carbomoylmethoxy]-2,8,14,20-tetrathiacalix[4]arene tetrabromide (cone 19) was synthesized according to the literature procedure. ${ }^{38}$

$5,11,17,23$-Tetra-tert-butyl-25,26,27,28-tetrakis[ $N$ - $\left(2^{\prime}, 2^{\prime}\right.$-diethyl-2'-\{([ethoxycarbonylmethyl]amidocarbonylmethyl)amidocarbonylmethyl\}ammoniumethyl)carbomoylmethoxy]-2,8,14,20tetrathiacalix[4]arene tetrabromide (cone 20) was synthesized according to the literature procedure. ${ }^{38}$

5,11,17,23-Tetra-tert-butyl-25,26,27,28-tetrakis[ $N$-(2', $2^{\prime}$-diethyl$2^{\prime}$-\{(ethoxycarbonyl[S-methyl]methyl)amidocarbonylmethyl $\}$ ammoniumethyl)carbomoylmethoxy]-2,8,14,20-tetrathiacalix[4] arene tetrabromide (cone 21) was synthesized according to the literature procedure. ${ }^{38}$

5,11,17,23-Tetra-tert-butyl-25,26,27,28-tetrakis $\left[N\right.$-( $\left(3^{\prime}, 3^{\prime}\right.$-dimethyl $-3^{\prime}-\{$ (ethoxycarbonylmethyl)amidocarbonylmethyl $\}$ ammoniumpropyl)carbomoylmethoxy]-2,8,14,20-tetrathiacalix[4]arene tetrabromide (1,3-alternate 27$)$ was synthesized according to the literature procedure. ${ }^{38}$

5,11,17,23-Tetra-tert-butyl-25,26,27,28-tetrakis[ $\left[N\right.$ - $\left(3^{\prime}, 3^{\prime}\right.$-dimethyl-3'-\{([ethoxycarbonylmethyl]amidocarbonylmethyl)amidocarbonylmethyl ammoniumpropyl)carbomoylmethoxy]-2,8,14,20tetrathiacalix[4]arene tetrabromide (1,3-alternate 28) was synthesized according to the literature procedure. ${ }^{38}$

5,11,17,23-Tetra-tert-butyl-25,26,27,28-tetrakis [ $N$-( $\left(3^{\prime}, 3^{\prime}\right.$-dimethyl-3'-\{(ethoxycarbonyl[S-methyl]methyl)amidocarbonylmethyl $\}$ ammoniumpropyl)carbomoylmethoxy]-2,8,14,20-tetrathiacalix [4]arene tetrabromide (1,3-alternate 29) was synthesized according to the literature procedure. ${ }^{38}$

$5,11,17,23$-Tetra-tert-butyl-25,26,27,28-tetrakis $\left[\left(\mathrm{N}\right.\right.$ - $\left(2^{\prime}, 2^{\prime}\right.$-diethyl-2' $-\{$ (ethoxycarbonylmethyl)amidocarbonylmethyl $\}$ )ammoniumethyl)carbomoylmethoxy]-2,8,14,20-tetrathiacalix[4]arene tetrabromide (1,3-alternate 36$)$ was synthesized according to the literature procedure. ${ }^{38}$

5,11,17,23-Tetra-tert-butyl-25,26,27,28-tetrakis[ $N$-( $2^{\prime}, 2^{\prime}$-diethyl$2^{\prime}-\{([$ ethoxycarbonylmethyl] amidocarbonylmethyl)amidocarbonylmethyl ammoniumethyl)carbomoylmethoxy]-2,8,14,20-tetrathiacalix[4]arene tetrabromide $(1,3$-alternate 37$)$ was synthesized according to the literature procedure. ${ }^{38}$

5,11,17,23-Tetra-tert-butyl-25,26,27,28-tetrakis[ $N$ - $\left(2^{\prime}, 2^{\prime}\right.$-diethyl-2'-\{(ethoxycarbonyl[S-methyl]methyl)amidocarbonylmethyl $\}$ ammoniumethyl)carbomoylmethoxy]-2,8,14,20-tetrathiacalix[4] arene tetrabromide (1,3-alternate 38$)$ was synthesized according to the literature procedure. ${ }^{38}$

General procedure for the synthesis of compounds $9,15-18$, 22-26, 31-35

The compounds 1-4 $\left(0.10 \mathrm{~g}, 0.08 \times 10^{-3} \mathrm{~mol}\right)$ were dissolved in $2 \mathrm{ml}$ of acetonitrile in the round bottom flask equipped with magnetic stirrer and a reflux condenser. Iodomethane, iodoethane, ethyl bromoacetate, pentyl bromoacetate, or benzyl bromide $\left(0.32 \times 10^{-3} \mathrm{~mol}\right)$ was added. The reaction mixture was refluxed for $48 \mathrm{~h}$. The solvent was removed under reduced pressure. The precipitate was dried under reduced pressure over phosphorus pentoxide.
$5,11,17,23-T e t r a-t e r t-b u t y l-25,26,27,28$-tetrakis $\left[N-\left(3^{\prime}, 3^{\prime}\right.\right.$-dimethyl-3'-(pentoxycarbonylmethyl)ammoniumpropyl)carbomoylmethoxy]-2,8,14,20-tetrathiacalix[4]arene tetrabromide (cone 9). Yield: $0.12 \mathrm{~g}(90 \%), \mathrm{mp} 106{ }^{\circ} \mathrm{C} .{ }^{1} \mathrm{H}$ NMR $(400 \mathrm{MHz}, 298 \mathrm{~K}$, $\left.\mathrm{CDCl}_{3}\right) \delta: 0.87\left(\mathrm{t},{ }^{3} J_{\mathrm{HH}}=6.9 \mathrm{~Hz}, 12 \mathrm{H}, \mathrm{CH}_{2} \mathrm{CH}_{3}\right), 1.08(\mathrm{~s}, 36 \mathrm{H}$, $\left.\left(\mathrm{CH}_{3}\right)_{3} \mathrm{C}\right), 1.27-1.31\left(\mathrm{~m}, 16 \mathrm{H}, \quad \mathrm{O}\left(\mathrm{CH}_{2}\right)_{2} \mathrm{CH}_{2} \mathrm{C}_{2} \mathrm{H}_{5}, \quad \mathrm{O}\left(\mathrm{CH}_{2}\right)_{3}-\right.$ $\left.\mathrm{CH}_{2} \mathrm{CH}_{3}\right), 1.60\left(\mathrm{~m}, 8 \mathrm{H}, \mathrm{OCH}_{2} \mathrm{CH}_{2} \mathrm{C}_{3} \mathrm{H}_{7}\right), 1.95\left(\mathrm{~m}, 8 \mathrm{H},-\mathrm{NCH}_{2}-\right.$ $\left.\mathrm{CH}_{2} \mathrm{CH}_{2} \mathrm{NH}\right), 3.22-3.25$ (s, 32H, $\left.\left(\mathrm{CH}_{3}\right)_{2} \mathrm{~N}^{+},-\mathrm{NCH}_{2} \mathrm{CH}_{2} \mathrm{CH}_{2} \mathrm{NH}\right)$, $3.60\left(\mathrm{~m}, 8 \mathrm{H}, \mathrm{NCH}_{2} \mathrm{CH}_{2} \mathrm{CH}_{2} \mathrm{NH}\right), 4.14\left(\mathrm{t},{ }^{3} J_{\mathrm{HH}}=6.9 \mathrm{~Hz}, 8 \mathrm{H}\right.$, $\left.\mathrm{OCH}_{2} \mathrm{C}_{4} \mathrm{H}_{9}\right), 4.51\left(\mathrm{~s}, 8 \mathrm{H}, \mathrm{N}^{+} \mathrm{CH}_{2} \mathrm{CO}\right), 4.82\left(\mathrm{~s}, 8 \mathrm{H}, \mathrm{OCH}_{2} \mathrm{CO}\right), 7.39$ (s, 8H, ArH), 8.56 (br. s, $4 \mathrm{H}, \mathrm{CONH}) .{ }^{13} \mathrm{C}$ NMR $(100 \mathrm{MHz}, 298 \mathrm{~K}$, DMSO-d $_{6}$ ) $\delta: 168.23,164.79,157.94,146.70,134.42,128.06$, $74.25,65.74,62.44,60.58,51.01,35.25,33.90,30.67,27.45$, 27.33, 22.53, 21.68, 13.82. El. anal. calcd for $\mathrm{C}_{96} \mathrm{H}_{156} \mathrm{Br}_{4} \mathrm{~N}_{8} \mathrm{O}_{16} \mathrm{~S}_{4}$ : C $54.23 \%, \mathrm{H} 7.40 \%$, N $5.27 \%$, S $6.03 \%$. Found: C $54.48 \%$, H $7.64 \%, \mathrm{~N} 5.52 \%$, S $6.24 \%$. MS (ESI): calcd for $\left[\mathrm{M}-2 \mathrm{Br}^{-}\right]^{2+} \mathrm{m} / \mathrm{z}=$ 983.2, $\left[\mathrm{M}-3 \mathrm{Br}^{-}\right]^{3+} m / z=628.8,\left[\mathrm{M}-4 \mathrm{Br}^{-}\right]^{4+} \mathrm{m} / z=451.6$, found $\mathrm{m} / \mathrm{z}=983.4,628.7,451.6$. IR $\nu_{\max }: 1663,1742(\mathrm{C}=\mathrm{O})$, 2956, $3349(\mathrm{NH})$.

$5,11,17,23-T e t r a-t e r t-b u t y l-25,26,27,28$-tetrakis $\left[\left(N-\left(2^{\prime}, 2^{\prime}, 2^{\prime}-\right.\right.\right.$ triethyl)ammoniumethyl)carbomoylmethoxy]-2,8,14,20-tetrathiacalix[4] arene tetraiodide (cone 15). Yield: $0.10 \mathrm{~g}(85 \%), \mathrm{mp}$ $163{ }^{\circ} \mathrm{C} .{ }^{1} \mathrm{H}$ NMR $\left(400 \mathrm{MHz}, 298 \mathrm{~K}, \mathrm{CDCl}_{3}\right) \delta: 1.11(\mathrm{~s}, 36 \mathrm{H}$, $\left.\left(\mathrm{CH}_{3}\right)_{3} \mathrm{C}\right), 1.41\left(\mathrm{t},{ }^{3} J_{\mathrm{HH}}=7.1 \mathrm{~Hz}, 36 \mathrm{H}, \mathrm{CH}_{3} \mathrm{CH}_{2}-\right), 3.57\left(\mathrm{q},{ }^{3} J_{\mathrm{HH}}=\right.$ $\left.7.1 \mathrm{~Hz}, 24 \mathrm{H},-\mathrm{CH}_{2} \mathrm{CH}_{3}\right), 3.66\left(\mathrm{~m}, 8 \mathrm{H},-\mathrm{NCH}_{2} \mathrm{CH}_{2} \mathrm{NH}\right), 3.94(\mathrm{~m}$, $\left.8 \mathrm{H}, \mathrm{NCH}_{2} \mathrm{CH}_{2} \mathrm{NH}\right), 4.99$ (s, 8H, $\mathrm{OCH}_{2} \mathrm{CO}$ ), 7.34 (s, 8H, ArH), 8.82 $\left(\mathrm{t},{ }^{3} \mathrm{HHH}_{\mathrm{HH}}=5.9 \mathrm{~Hz}, 4 \mathrm{H}, \mathrm{CONH}\right) .{ }^{13} \mathrm{C}$ NMR $(100 \mathrm{MHz}, 298 \mathrm{~K}$, DMSO$\left.\mathrm{d}_{6}\right) \delta: 169.76,157.13,147.73,134.91,128.17,73.99,55.82,54.15$, 34.31, 33.68, 31.11, 8.29. El. anal. calcd for $\mathrm{C}_{80} \mathrm{H}_{132} \mathrm{I}_{4} \mathrm{~N}_{8} \mathrm{O}_{8} \mathrm{~S}_{4}: \mathrm{C}$ $48.78 \%, \mathrm{H} 6.75 \%$, N 5.69\%, S 6.51\%. Found: C 48.59\%, H $6.66 \%, \mathrm{~N} 5.51 \%$, S $6.42 \%$. MS (ESI): calcd for $\left[\mathrm{M}-2 \mathrm{I}^{-}\right]^{2+} \mathrm{m} / \mathrm{z}=$ 858.0, $\left[\mathrm{M}-3 \mathrm{I}^{-}\right]^{3+} \mathrm{m} / \mathrm{z}=529.7,\left[\mathrm{M}-4 \mathrm{I}^{-}\right]^{4+} \mathrm{m} / \mathrm{z}=365.5$, found $m / z=857.9$, 529.6, 365.4. IR $\nu_{\text {max }}: 1669(\mathrm{C}=\mathrm{O}), 2955,3317(\mathrm{NH})$.

$5,11,17,23-T e t r a-t e r t-b u t y l-25,26,27,28$-tetrakis $\left[\left(N\right.\right.$ - $\left(2^{\prime}, 2^{\prime}\right.$-diethyl-2'-benzyl)ammoniumethyl)carbomoylmethoxy]-2,8,14,20tetrathiacalix[4]arene tetrabromide (cone 16). Yield: $0.12 \mathrm{~g}$ (91\%), mp $139^{\circ} \mathrm{C} .{ }^{1} \mathrm{H}$ NMR (400 MHz, $298 \mathrm{~K}, \mathrm{CDCl}_{3}$ ) $\delta: 1.10(\mathrm{~s}$, $\left.36 \mathrm{H},\left(\mathrm{CH}_{3}\right)_{3} \mathrm{C}\right), 1.44\left(\mathrm{t},{ }^{3} J_{\mathrm{HH}}=6.7 \mathrm{~Hz}, 24 \mathrm{H}, \mathrm{CH}_{3} \mathrm{CH}_{2}-\right), 3.50$ (q, $\left.{ }^{3} J_{\mathrm{HH}}=6.7 \mathrm{~Hz}, 16 \mathrm{H},-\mathrm{CH}_{2} \mathrm{CH}_{3}\right), 3.63\left(\mathrm{~m}, 8 \mathrm{H},-\mathrm{NCH}_{2} \mathrm{CH}_{2} \mathrm{NH}\right)$, $4.10\left(\mathrm{~m}, 8 \mathrm{H}, \mathrm{NCH}_{2} \mathrm{CH}_{2} \mathrm{NH}\right), 4.92\left(\mathrm{~s}, 8 \mathrm{H}, \mathrm{N}^{+} \mathrm{CH}_{2} \mathrm{Ph}\right), 5.03(\mathrm{~s}, 8 \mathrm{H}$, $\left.\mathrm{OCH}_{2} \mathrm{CO}\right), 7.32(\mathrm{~s}, 8 \mathrm{H}, \mathrm{ArH}), 7.36-7.40\left(\mathrm{~m}, 8 \mathrm{H}, \mathrm{Ar}^{\prime} \mathrm{H}\right), 7.64(\mathrm{~m}$, $\left.12 \mathrm{H}, \mathrm{Ar}^{\prime} \mathrm{H}\right), 9.08\left(\mathrm{t},{ }^{3} \mathrm{JHH}_{\mathrm{HH}}=5.7 \mathrm{~Hz}, 4 \mathrm{H}, \mathrm{CONH}\right) .{ }^{13} \mathrm{C}$ NMR $(100$ $\left.\mathrm{MHz}, 298 \mathrm{~K}, \mathrm{CDCl}_{3}\right) \delta: 169.89,157.44,147.45,134.85,132.89$, 130.61, 129.38, 128.30, 127.09, 73.98, 62.04, 55.80, 53.91, 34.27, 33.72, 31.11, 8.70. El. anal. calcd for $\mathrm{C}_{100} \mathrm{H}_{140} \mathrm{Br}_{4} \mathrm{~N}_{8} \mathrm{O}_{8} \mathrm{~S}_{4}$ : C $59.16 \%, \mathrm{H}$ 6.95\%, N 5.52\%, S 6.32\%. Found: C 59.35\%, H $6.72 \%$, N $5.23 \%$, S $6.47 \%$. MS (ESI): calcd for $\left[\mathrm{M}-2 \mathrm{Br}^{-}\right]^{2+} \mathrm{m} / \mathrm{z}=$ 935.2, $\left[\mathrm{M}-3 \mathrm{Br}^{-}\right]^{3+} \mathrm{m} / z=596.8,\left[\mathrm{M}-4 \mathrm{Br}^{-}\right]^{4+} \mathrm{m} / z=427.6$, found $m / z=935.4,596.7,427.7$. IR $\nu_{\text {max }}: 1670(\mathrm{C}=\mathrm{O}), 2960$, $3320(\mathrm{NH})$.

$5,11,17,23$-Tetra-tert-butyl-25,26,27,28-tetrakis $\left[N\right.$ - $\left(2^{\prime}, 2^{\prime}\right.$-diethyl-2'-(ethoxycarbonylmethyl)ammoniumethyl)carbomoylmethoxy]-2,8,14,20-tetrathiacalix[4]arene tetrabromide (cone 17). Yield: $0.12 \mathrm{~g}(90 \%), \mathrm{mp} 118{ }^{\circ} \mathrm{C} .{ }^{1} \mathrm{H}$ NMR $(400 \mathrm{MHz}, 298 \mathrm{~K}$, $\left.\mathrm{CDCl}_{3}\right) \delta: 1.10\left(\mathrm{~s}, 36 \mathrm{H},\left(\mathrm{CH}_{3}\right)_{3} \mathrm{C}\right), 1.29\left(\mathrm{t},{ }^{3} J_{\mathrm{HH}}=7.1 \mathrm{~Hz}, 12 \mathrm{H}\right.$ $\left.\mathrm{OCH}_{2} \mathrm{CH}_{3}\right), 1.46\left(\mathrm{t},{ }^{3} \mathrm{HH}_{\mathrm{HH}}=7.0 \mathrm{~Hz}, 24 \mathrm{H}, \mathrm{CH}_{3} \mathrm{CH}_{2}-\right), 3.88-4.03(\mathrm{~m}$, 
$32 \mathrm{H},-\mathrm{CH}_{2} \mathrm{CH}_{3},-\mathrm{NCH}_{2} \mathrm{CH}_{2} \mathrm{NH}, 8 \mathrm{H}, \mathrm{NCH}_{2} \mathrm{CH}_{2} \mathrm{NH}$ ), 4.24 (q, $\left.{ }^{3} J_{\mathrm{HH}}=7.1 \mathrm{~Hz}, 8 \mathrm{H}, \mathrm{OCH}_{2} \mathrm{CH}_{3}\right) .4 .75\left(\mathrm{~s}, 8 \mathrm{H}, \mathrm{N}^{+} \mathrm{CH}_{2} \mathrm{CO}\right), 4.97(\mathrm{~s}$, $8 \mathrm{H}, \mathrm{OCH}_{2} \mathrm{CO}$ ), 7.33 (s, 8H, ArH), 9.03 (br. s, $\left.4 \mathrm{H}, \mathrm{CONH}\right) .{ }^{13} \mathrm{C}$ NMR (100 MHz, $\left.298 \mathrm{~K}, \mathrm{CDCl}_{3}\right) \delta: 169.97,164.50,157.34,147.56$, 134.84, 128.27, 73.95, 62.90, 57.49, 56.71, 56.23, 34.26, 33.71, 31.08, 14.02, 8.56. El. anal. calcd for $\mathrm{C}_{88} \mathrm{H}_{140} \mathrm{Br}_{4} \mathrm{~N}_{8} \mathrm{O}_{16} \mathrm{~S}_{4}$ : C $52.48 \%, \mathrm{H} 6.53 \%, \mathrm{~N} 5.86 \%$, S 6.70\%. Found: C 52.65\%, H $6.71 \%$, N 6.01\%, S 6.27\%. MS (ESI): calcd for $\left[\mathrm{M}-3 \mathrm{Br}^{-}\right]^{3+} \mathrm{m} / z=$ 591.3, $\left[\mathrm{M}-4 \mathrm{Br}^{-}\right]^{4+} m / z=423.5$, found $m / z=591.5$, 423.5. IR $\nu_{\text {max }}: 1666,1740(\mathrm{C}=\mathrm{O}), 2958,3322(\mathrm{NH})$.

$5,11,17,23-T e t r a-t e r t-b u t y l-25,26,27,28-t e t r a k i s\left[N-\left(2^{\prime}, 2^{\prime}\right.\right.$-diethyl2 -(pentoxycarbonylmethyl)ammoniumethyl)carbomoylmethoxy]2,8,14,20-tetrathiacalix[4]arene tetrabromide (cone 18). Yield: $0.11 \mathrm{~g}(90 \%), \operatorname{mp} 135{ }^{\circ} \mathrm{C} .{ }^{1} \mathrm{H}$ NMR $\left(400 \mathrm{MHz}, 298 \mathrm{~K}, \mathrm{CDCl}_{3}\right) \delta$ : $0.88\left(\mathrm{t},{ }^{3} \mathrm{~J}_{\mathrm{HH}}=6.8 \mathrm{~Hz}, 12 \mathrm{H}, \mathrm{O}\left(\mathrm{CH}_{2}\right)_{4} \mathrm{CH}_{3}\right), 1.09\left(\mathrm{~s}, 36 \mathrm{H},\left(\mathrm{CH}_{3}\right)_{3} \mathrm{C}\right)$, 1.26-1.33 (m, 16H, O $\left.\left(\mathrm{CH}_{2}\right)_{3} \mathrm{CH}_{2} \mathrm{CH}_{3}, \mathrm{O}\left(\mathrm{CH}_{2}\right)_{2} \mathrm{CH}_{2} \mathrm{C}_{2} \mathrm{H}_{5}\right), 1.44(\mathrm{t}$, $\left.{ }^{3} J_{\mathrm{HH}}=7.1 \mathrm{~Hz}, 24 \mathrm{H}, \mathrm{CH}_{3} \mathrm{CH}_{2} \mathrm{~N}^{+}\right), 1.63\left(\mathrm{~m}, 8 \mathrm{H}, \mathrm{OCH}_{2} \mathrm{CH}_{2} \mathrm{C}_{3} \mathrm{H}_{7}\right)$, 3.88-3.97 (m, $\left.32 \mathrm{H},-\mathrm{N}^{+} \mathrm{CH}_{2} \mathrm{CH}_{3},-\mathrm{NCH}_{2} \mathrm{CH}_{2} \mathrm{NH},-\mathrm{NCH}_{2} \mathrm{CH}_{2} \mathrm{NH}\right)$, $4.14\left(\mathrm{t},{ }^{3} J_{\mathrm{HH}}=6.8 \mathrm{~Hz}, 8 \mathrm{H}, \mathrm{OCH}_{2} \mathrm{C}_{4} \mathrm{H}_{9}\right), 4.70\left(\mathrm{~s}, 8 \mathrm{H}, \mathrm{N}^{+} \mathrm{CH}_{2} \mathrm{CO}\right)$, 4.93 (s, 8H, $\mathrm{OCH}_{2} \mathrm{CO}$ ), 7.30 (s, 8H, ArH), 9.01 (br. s, 4H, CONH). ${ }^{13} \mathrm{C}$ NMR $\left(100 \mathrm{MHz}, 298 \mathrm{~K}, \mathrm{CDCl}_{3}\right) \delta: 169.88,164.55,157.40$, 147.51, 134.82, 128.22, 73.90, 66.91, 57.63, 56.76, 55.91, 34.25, $33.81,31.09,27.97,27.75,22.90,13.91,8.78$. El. anal. calcd for $\mathrm{C}_{100} \mathrm{H}_{164} \mathrm{Br}_{4} \mathrm{~N}_{8} \mathrm{O}_{16} \mathrm{~S}_{4}$ : C 55.04\%, H 7.57\%, N 5.13\%, S 5.88\%. Found: C 54.99\%, H 7.53\%, N 4.98\%, S 5.63\%. MS (ESI): calcd for $\left[\mathrm{M}-4 \mathrm{Br}^{-}\right]^{4+} m / z=465.6$, found $m / z=465.6$. IR $\nu_{\text {max }}: 1670$, $1741(\mathrm{C}=\mathrm{O}), 2956,3320(\mathrm{NH})$.

$5,11,17,23$-Tetra-tert-butyl-25,26,27, 28-tetrakis $\left[\left(N-\left(3^{\prime}, 3^{\prime}, 3^{\prime}-\right.\right.\right.$ trimethyl)ammoniumpropyl)carbomoylmethoxy]-2,8,14,20tetrathiacalix[4]arene tetraiodide (1,3-alternate 22). Yield: $0.12 \mathrm{~g}$ (90\%), mp $197{ }^{\circ} \mathrm{C} .{ }^{1} \mathrm{H}$ NMR (400 MHz, $298 \mathrm{~K}, \mathrm{DMSO}_{\mathrm{d}}$ ) $\delta: 1.21$ $\left(\mathrm{s}, 36 \mathrm{H},\left(\mathrm{CH}_{3}\right)_{3} \mathrm{C}\right), 1.91\left(\mathrm{~m}, 8 \mathrm{H},-\mathrm{NCH}_{2} \mathrm{CH}_{2} \mathrm{CH}_{2} \mathrm{NH}\right), 3.17$ (s, 36H, $\left.\left(\mathrm{CH}_{3}\right)_{3} \mathrm{~N}^{+}\right), 3.32\left(\mathrm{~m}, 8 \mathrm{H},-\mathrm{NCH}_{2} \mathrm{CH}_{2} \mathrm{CH}_{2} \mathrm{NH}\right), 3.60(\mathrm{~m}, 8 \mathrm{H}$, $\mathrm{NCH}_{2} \mathrm{CH}_{2} \mathrm{CH}_{2} \mathrm{NH}$ ), 3.98 (s, 8H, $\mathrm{OCH}_{2} \mathrm{CO}$ ), 7.60 (s, 8H, ArH), 8.01 $\left(\mathrm{t},{ }^{3} \mathrm{~J}_{\mathrm{HH}}=5.2 \mathrm{~Hz}, 4 \mathrm{H}, \mathrm{CONH}\right) .{ }^{13} \mathrm{C}$ NMR $(100 \mathrm{MHz}, 298 \mathrm{~K}$, DMSO$\left.\mathrm{d}_{6}\right) \delta: 167.37,157.02,146.08,132.95,127.61,70.96,63.23,52.23$, $35.81,33.89,30.78,22.86$. El. anal. calcd for $\mathrm{C}_{72} \mathrm{H}_{116} \mathrm{I}_{4} \mathrm{~N}_{8} \mathrm{O}_{8} \mathrm{~S}_{4}: \mathrm{C}$ $46.55 \%, \mathrm{H} 6.29 \%$, N 6.03\%, S 6.90\%. Found: C $46.40 \%, \mathrm{H}$ $5.89 \%$, N 5.60\%, S 6.00\%. MS (MALDI TOF): calcd for $\left[\mathrm{M}-\mathrm{I}^{-}\right]^{+}$ $m / z=1730.7$, found $m / z=1730.2$. IR $\nu_{\max }: 1660(\mathrm{C}=\mathrm{O}), 2955$, 3292 (NH).

$5,11,17,23-T e t r a-t e r t$-butyl-25,26,27,28-tetrakis $\left[\left(N-\left(3^{\prime}, 3^{\prime}\right.\right.\right.$-dimethyl-3'-ethyl)ammoniumpropyl)carbomoylmethoxy]-2,8,14,20tetrathiacalix[4] arene tetraiodide (1,3-alternate 23). Yield: $0.14 \mathrm{~g}$ (95\%), mp $215{ }^{\circ} \mathrm{C} .{ }^{1} \mathrm{H}$ NMR (400 MHz, $298 \mathrm{~K}, \mathrm{DMSO}_{6}$ ) $\delta: 1.21$ $\left(\mathrm{s}, 36 \mathrm{H},\left(\mathrm{CH}_{3}\right)_{3} \mathrm{C}\right), 1.24\left(\mathrm{t},{ }^{3} J_{\mathrm{HH}}=7.1 \mathrm{~Hz}, 12 \mathrm{H}, \mathrm{N}^{+} \mathrm{CH}_{2} \mathrm{CH}_{3}\right), 1.88$ $\left(\mathrm{m}, 8 \mathrm{H},-\mathrm{NCH}_{2} \mathrm{CH}_{2} \mathrm{CH}_{2} \mathrm{NH}\right), 3.01\left(\mathrm{~s}, 24 \mathrm{H},\left(\mathrm{CH}_{3}\right)_{2} \mathrm{~N}^{+}\right), 3.19(\mathrm{~m}$, $\left.8 \mathrm{H},-\mathrm{NCH}_{2} \mathrm{CH}_{2} \mathrm{CH}_{2} \mathrm{NH}\right), 3.29\left(\mathrm{~m}, 8 \mathrm{H}, \mathrm{NCH}_{2} \mathrm{CH}_{2} \mathrm{CH}_{2} \mathrm{NH}\right), 3.35$ $\left(\mathrm{q},{ }^{3} J_{\mathrm{HH}}=7.2 \mathrm{~Hz}, 8 \mathrm{H}, \mathrm{N}^{+} \mathrm{CH}_{2} \mathrm{CH}_{3}\right), 3.99\left(\mathrm{~s}, 8 \mathrm{H}, \mathrm{OCH}_{2} \mathrm{CO}\right), 7.60$ (s, $8 \mathrm{H}, \mathrm{ArH}), 8.03$ (br. s, 4H, CONH). ${ }^{13} \mathrm{C}$ NMR (100 MHz, $298 \mathrm{~K}$, DMSO-d $\left.{ }_{6}\right) \delta: 167.37,157.06,146.04,133.00,127.56,70.84,60.23$, $58.59,49.60,35.80,33.88,30.78,22.44,7.84$. El. anal. calcd for $\mathrm{C}_{76} \mathrm{H}_{124} \mathrm{I}_{4} \mathrm{~N}_{8} \mathrm{O}_{8} \mathrm{~S}_{4}$ : C 47.70\%, H 6.53\%, N 5.86\%, S 6.70\%. Found: C $47.53 \%$, H 6.52\%, N 5.56\%, S 6.64\%. MS (MALDI TOF): calcd for $\left[\mathrm{M}-\mathrm{I}^{-}\right]^{+} m / z=1786.8$, found $m / z=1786.5$. IR $\nu_{\text {max }}: 1653$ $(\mathrm{C}=\mathrm{O}), 2958,3335(\mathrm{NH})$.
$5,11,17,23$-Tetra-tert-butyl-25,26,27,28-tetrakis $\left[\left(N\right.\right.$ - $\left(3^{\prime}, 3^{\prime}\right.$-dimethyl-3'-benzyl)ammoniumpropyl)carbomoylmethoxy]-2,8,14,20tetrathiacalix[4]arene tetrabromide (1,3-alternate 24). Yield: $0.12 \mathrm{~g}$ (91\%), mp $150{ }^{\circ} \mathrm{C} .{ }^{1} \mathrm{H}$ NMR (400 MHz, $298 \mathrm{~K}$, DMSO-d $\left.{ }_{6}\right) \delta$ : $1.20\left(\mathrm{~s}, 36 \mathrm{H},\left(\mathrm{CH}_{3}\right)_{3} \mathrm{C}\right), 2.05\left(\mathrm{~m}, 8 \mathrm{H},-\mathrm{NCH}_{2} \mathrm{CH}_{2} \mathrm{CH}_{2} \mathrm{NH}\right), 3.00(\mathrm{~s}$, $\left.24 \mathrm{H},\left(\mathrm{CH}_{3}\right)_{2} \mathrm{~N}^{+}\right), 3.21\left(\mathrm{~m}, 8 \mathrm{H},-\mathrm{NCH}_{2} \mathrm{CH}_{2} \mathrm{CH}_{2} \mathrm{NH}\right), 3.37(\mathrm{~m}, 8 \mathrm{H}$, $\left.\mathrm{NCH}_{2} \mathrm{CH}_{2} \mathrm{CH}_{2} \mathrm{NH}\right), 4.01\left(\mathrm{~s}, 8 \mathrm{H}, \mathrm{OCH}_{2} \mathrm{CO}\right), 4.60(\mathrm{~s}, 8 \mathrm{H}$, $\mathrm{N}^{+} \mathrm{CH}_{2} \mathrm{Ph}$ ), $7.52\left(\mathrm{~m}, 12 \mathrm{H}, \mathrm{Ar}^{\prime} \mathrm{H}\right), 7.59\left(\mathrm{~m}, 8 \mathrm{H}, \mathrm{Ar}^{\prime} \mathrm{H}\right), 7.60(\mathrm{~s}, 8 \mathrm{H}$, ArH), 8.10 (br. s, 4H, CONH). ${ }^{13} \mathrm{C}$ NMR (100 MHz, $298 \mathrm{~K}$, DMSO$\left.\mathrm{d}_{6}\right) \delta: 167.44,157.03,146.06,133.12,132.95,130.29,128.89$, 128.00, 127.57, 70.90, 65.91, 61.12, 49.22, 35.86, 33.87, 30.79, 22.63. El. anal. calcd for $\mathrm{C}_{96} \mathrm{H}_{132} \mathrm{Br}_{4} \mathrm{~N}_{8} \mathrm{O}_{8} \mathrm{~S}_{4}$ : C 58.41\%, H 6.74\%, $\mathrm{N} 5.68 \%$, S 6.50\%. Found: C 58.75\%, H 6.62\%, N 6.01\%, S 6.37\%. MS (MALDI TOF): calcd for $\left[\mathrm{M}-\mathrm{Br}^{-}\right]^{+} \mathrm{m} / z=1245.7$, found $m / z=1245.8$. IR $\nu_{\text {max }}: 1664(\mathrm{C}=\mathrm{O}), 2955,3287(\mathrm{NH})$.

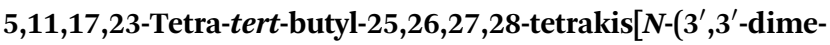
thyl-3'-(ethoxycarbonylmethyl)ammoniumpropyl)carbomoylmethoxy]-2,8,14,20-tetrathiacalix[4] arene tetrabromide (1,3alternate 25). Yield: $0.13 \mathrm{~g}$ (92\%), mp $123{ }^{\circ} \mathrm{C} .{ }^{1} \mathrm{H} \mathrm{NMR}(400 \mathrm{MHz}$, $\left.298 \mathrm{~K}, \mathrm{DMSO}_{6}\right) \delta: 1.20\left(\mathrm{~s}, 36 \mathrm{H},\left(\mathrm{CH}_{3}\right)_{3} \mathrm{C}\right), 1.25\left(\mathrm{t},{ }^{3} J_{\mathrm{HH}}=7.1 \mathrm{~Hz}\right.$, $\left.12 \mathrm{H}, \mathrm{OCH}_{2} \mathrm{CH}_{3}\right) 1.91\left(\mathrm{~m}, 8 \mathrm{H},-\mathrm{NCH}_{2} \mathrm{CH}_{2} \mathrm{CH}_{2} \mathrm{NH}\right), 3.17(\mathrm{~m}, 8 \mathrm{H}$, $\left.-\mathrm{NCH}_{2} \mathrm{CH}_{2} \mathrm{CH}_{2} \mathrm{NH}\right), 3.22\left(\mathrm{~s}, 24 \mathrm{H},\left(\mathrm{CH}_{3}\right)_{2} \mathrm{~N}^{+}\right), 4.00(\mathrm{~s}, 8 \mathrm{H}$, $\left.\mathrm{OCH}_{2} \mathrm{CO}\right), 4.55\left(\mathrm{~m}, 8 \mathrm{H}, \mathrm{NCH}_{2} \mathrm{CH}_{2} \mathrm{CH}_{2} \mathrm{NH}\right), 4.24\left(\mathrm{q},{ }^{3} J_{\mathrm{HH}}=\right.$ $\left.7.2 \mathrm{~Hz}, 8 \mathrm{H}, \mathrm{N}^{+} \mathrm{CH}_{2} \mathrm{CH}_{3}\right) .4 .47\left(\mathrm{~s}, 8 \mathrm{H}, \mathrm{N}^{+} \mathrm{CH}_{2} \mathrm{CO}\right), 7.60(\mathrm{~s}, 8 \mathrm{H}$, ArH), 8.05 (br. s, 4H, CONH). ${ }^{13} \mathrm{C}$ NMR (100 MHz, 298 K, DMSO$\left.\mathrm{d}_{6}\right) \delta: 167.39,164.85,157.40,146.19,133.26,127.82,70.89$, $62.30,62.00,60.54,51.12,35.69,33.85,30.77,22.44,13.85$. El. anal. calcd for $\mathrm{C}_{84} \mathrm{H}_{132} \mathrm{Br}_{4} \mathrm{~N}_{8} \mathrm{O}_{16} \mathrm{~S}_{4}$ : C 51.53\%, H 6.80\%, N 5.72\%, S 6.55\%. Found: C 51.49\%, H 6.58\%, N 5.55\%, S 6.64\%. MS (ESI): calcd for $\left[\mathrm{M}-4 \mathrm{Br}^{-}\right]^{4+} \mathrm{m} / z=409.6$, found $\mathrm{m} / z=409.5$. IR $\nu_{\text {max }}$ : 1666, $1742(\mathrm{C}=\mathrm{O}), 2958,3300(\mathrm{NH})$.

$5,11,17,23$-Tetra-tert-butyl-25,26,27,28-tetrakis[ $N$-( $3^{\prime}, 3^{\prime}$-dimethyl-3' -(pentoxycarbonylmethyl)ammoniumpropyl)carbomoylmethoxy]-2,8,14,20-tetrathiacalix[4] arene tetrabromide (1,3alternate 26). Yield: $0.13 \mathrm{~g}(92 \%), \mathrm{mp} 103{ }^{\circ} \mathrm{C} .{ }^{1} \mathrm{H} \mathrm{NMR}(400 \mathrm{MHz}$, $\left.298 \mathrm{~K}, \mathrm{DMSO}_{6}\right) \delta: 0.87\left(\mathrm{t},{ }^{3} \mathrm{~J}_{\mathrm{HH}}=6.9 \mathrm{~Hz}, 12 \mathrm{H} \mathrm{CH}_{2} \mathrm{CH}_{3}\right), 1.20(\mathrm{~s}$, $\left.36 \mathrm{H},\left(\mathrm{CH}_{3}\right)_{3} \mathrm{C}\right), 1.27-1.33\left(\mathrm{~m}, 16 \mathrm{H}, \mathrm{O}\left(\mathrm{CH}_{2}\right)_{2} \mathrm{CH}_{2} \mathrm{C}_{2} \mathrm{H}_{5}, \mathrm{O}\left(\mathrm{CH}_{2}\right)_{3}-\right.$ $\left.\mathrm{CH}_{2} \mathrm{CH}_{3}\right), 1.62\left(\mathrm{~m}, 8 \mathrm{H}, \mathrm{OCH}_{2} \mathrm{CH}_{2} \mathrm{C}_{3} \mathrm{H}_{7}\right), 1.92\left(\mathrm{~m}, 8 \mathrm{H},-\mathrm{NCH}_{2}-\right.$ $\left.\mathrm{CH}_{2} \mathrm{CH}_{2} \mathrm{NH}\right), 3.18\left(\mathrm{~m}, 8 \mathrm{H},-\mathrm{NCH}_{2} \mathrm{CH}_{2} \mathrm{CH}_{2} \mathrm{NH}\right), 3.35$ (s, $24 \mathrm{H}$, $\left.\left(\mathrm{CH}_{3}\right)_{2} \mathrm{~N}^{+}\right), 3.56\left(\mathrm{~m}, 8 \mathrm{H}, \mathrm{NCH}_{2} \mathrm{CH}_{2} \mathrm{CH}_{2} \mathrm{NH}\right), 3.98(\mathrm{~s}, 8 \mathrm{H}$, $\left.\mathrm{OCH}_{2} \mathrm{CO}\right), 4.17\left(\mathrm{t},{ }^{3} \mathrm{~J}_{\mathrm{HH}}=6.6 \mathrm{~Hz}, 4 \mathrm{H}, \mathrm{OCH}_{2} \mathrm{C}_{4} \mathrm{H}_{9}\right) 4.50(\mathrm{~s}, 8 \mathrm{H}$, $\mathrm{N}^{+} \mathrm{CH}_{2} \mathrm{CO}$ ), $7.60(\mathrm{~s}, 8 \mathrm{H}, \mathrm{ArH}), 8.06\left(\mathrm{t},{ }^{3} J_{\mathrm{HH}}=5.4 \mathrm{~Hz}, 4 \mathrm{H}, \mathrm{CONH}\right)$. ${ }^{13} \mathrm{C}$ NMR (100 MHz, $298 \mathrm{~K}$, DMSO-d 6 ) $\delta: 167.37,164.77,157.10$, 146.01, 133.03, 127.54, 70.94, 65.80, 62.36, 60.53, 51.12, 35.70, $33.84,30.78,27.44,27.36,22.55,21.66,13.79$. El. anal. calcd for $\mathrm{C}_{96} \mathrm{H}_{156} \mathrm{Br}_{4} \mathrm{~N}_{8} \mathrm{O}_{16} \mathrm{~S}_{4}:$ C 54.23\%, H 7.40\%, N 5.27\%, S 6.03\%. Found: C 54.12\%, H 7.54\%, N 5.38\%, S 5.96\%. MS (ESI): calcd for $\left[\mathrm{M}-2 \mathrm{Br}^{-}\right]^{2+} m / z=983.2,\left[\mathrm{M}-3 \mathrm{Br}^{-}\right]^{3+} m / z=628.8,[\mathrm{M}-$ $\left.4 \mathrm{Br}^{-}\right]^{4+} m / z=451.6$, found $m / z=982.4,628.9$, 451.6. IR $\nu_{\max }$ : 1665, $1742(\mathrm{C}=\mathrm{O}), 2954,3300(\mathrm{NH})$.

$5,11,17,23-T e t r a-t e r t-b u t y l-25,26,27,28-t e t r a k i s\left[\left(N-\left(2^{\prime}\right.\right.\right.$-methyl$2^{\prime}, 2^{\prime}$-diethyl)ammoniumethyl)carbomoylmethoxy]-2,8,14,20tetrathiacalix[4] arene tetraiodide (1,3-alternate 31). Yield: $0.13 \mathrm{~g}$ (95\%), mp $156{ }^{\circ} \mathrm{C} .{ }^{1} \mathrm{H}$ NMR (400 MHz, $298 \mathrm{~K}, \mathrm{DMSO}_{6}$ ) $\delta: 1.22$ $\left(\mathrm{s}, 36 \mathrm{H},\left(\mathrm{CH}_{3}\right)_{3} \mathrm{C}\right), 1.27\left(\mathrm{t},{ }^{3} J_{\mathrm{HH}}=6.5 \mathrm{~Hz}, 24 \mathrm{H},\left(\mathrm{CH}_{3} \mathrm{CH}_{2}-\right)\right), 2.98$ $\left(\mathrm{s}, 12 \mathrm{H},\left(\mathrm{CH}_{3}\right) \mathrm{N}^{+}\right), 3.21\left(\mathrm{~m}, 8 \mathrm{H},-\mathrm{NCH}_{2} \mathrm{CH}_{2} \mathrm{NH}\right), 3.39\left(\mathrm{q},{ }^{3} J_{\mathrm{HH}}=\right.$ 
$\left.6.5 \mathrm{~Hz}, 16 \mathrm{H},-\mathrm{CH}_{2} \mathrm{CH}_{3}\right), 3.49\left(\mathrm{~m}, 8 \mathrm{H}, \mathrm{NCH}_{2} \mathrm{CH}_{2} \mathrm{NH}\right), 4.05(\mathrm{~s}, 8 \mathrm{H}$, $\mathrm{OCH}_{2} \mathrm{CO}$ ), 7.61 (s, 8H, ArH), $8.18\left(\mathrm{t},{ }^{3} J_{\mathrm{HH}}=5.5 \mathrm{~Hz}, 4 \mathrm{H}, \mathrm{CONH}\right)$. ${ }^{13} \mathrm{C}$ NMR (100 MHz, $298 \mathrm{~K}$, DMSO-d 6 ) $\delta: 168.03,156.80,146.08$, 132.95, 127.51, 76.13, 70.51, 65.99, 62.12, 56.83, 56.16, 47.02, $33.91,32.13,30.87,26.70,25.36,7.46$. El. anal. calcd for $\mathrm{C}_{76} \mathrm{H}_{124} \mathrm{I}_{4} \mathrm{~N}_{8} \mathrm{O}_{8} \mathrm{~S}_{4}:$ C $47.70 \%, \mathrm{H} 6.53 \%$, N 5.86\%, S 6.70\%. Found: C $47.54 \%$, H 5.93\%, N 6.16\%, S 6.17\%. MS (ESI): calcd for [M $\left.2 \mathrm{I}^{-}\right]^{2+} m / z=829.3,\left[\mathrm{M}-3 \mathrm{I}^{-}\right]^{3+} m / z=510.3,\left[\mathrm{M}-4 \mathrm{I}^{-}\right]^{4+} \mathrm{m} / z=$ 351.2 , found $m / z=829.3,510.6$, 351.4. IR $\nu_{\text {max }}: 1664(\mathrm{C}=\mathrm{O})$, 2955, $3300(\mathrm{NH})$.

$5,11,17,23-$ Tetra-tert-butyl-25,26,27,28-tetrakis $\left[\left(N-\left(2^{\prime}, 2^{\prime}, 2^{\prime}-\right.\right.\right.$ triethyl)ammoniumethyl)carbomoylmethoxy]-2,8,14,20-tetrathiacalix[4] arene tetraiodide (1,3-alternate 32$)$. Yield: $0.11 \mathrm{~g}$ (87\%), mp $173{ }^{\circ} \mathrm{C} .{ }^{1} \mathrm{H}$ NMR (400 MHz, $298 \mathrm{~K}, \mathrm{DMSO}_{6}$ ) $\delta: 1.22$ $\left(\mathrm{s}, 36 \mathrm{H},\left(\mathrm{CH}_{3}\right)_{3} \mathrm{C}\right), 1.23\left(\mathrm{t},{ }^{3} J_{\mathrm{HH}}=7.1 \mathrm{~Hz}, 36 \mathrm{H}, \mathrm{CH}_{3} \mathrm{CH}_{2}-\right), 3.15$ $\left(\mathrm{m}, 8 \mathrm{H},-\mathrm{NCH}_{2} \mathrm{CH}_{2} \mathrm{NH}\right), 3.31\left(\mathrm{q},{ }^{3} J_{\mathrm{HH}}=7.1 \mathrm{~Hz}, 24 \mathrm{H},-\mathrm{CH}_{2} \mathrm{CH}_{3}\right)$, $3.47\left(\mathrm{~m}, 8 \mathrm{H}, \mathrm{NCH}_{2} \mathrm{CH}_{2} \mathrm{NH}\right), 4.03$ (s, 8H, $\left.\mathrm{OCH}_{2} \mathrm{CO}\right), 7.61$ (s, 8H, $\mathrm{ArH}), 8.13\left(\mathrm{t},{ }^{3} J_{\mathrm{HH}}=5.3 \mathrm{~Hz}, 4 \mathrm{H}, \mathrm{CONH}\right) .{ }^{13} \mathrm{C} \mathrm{NMR}(100 \mathrm{MHz}, 298$ K, DMSO-d ${ }_{6}$ ) $\delta: 167.98,156.74,146.14,132.94,127.58,70.46$, 53.53, 52.46, 33.91, 31.84, 30.84, 7.04. El. anal. calcd for $\mathrm{C}_{80} \mathrm{H}_{132} \mathrm{I}_{4} \mathrm{~N}_{8} \mathrm{O}_{8} \mathrm{~S}_{4}$ : C 48.78\%, H 6.75\%, N 5.69\%, S 6.51\%. Found: C $49.02 \%$, H 6.58\%, N 5.73\%, S 6.37\%. MS (ESI): calcd for [M $\left.4 \mathrm{I}^{-}\right]^{4+} m / z=365.6$, found $m / z=365.5$. IR $\nu_{\max }: 1664(\mathrm{C}=\mathrm{O})$, 2955, $3261(\mathrm{NH})$.

$5,11,17,23$-Tetra-tert-butyl-25,26,27,28-tetrakis $\left[N\right.$-( $2^{\prime}, 2^{\prime}$-diethyl$2^{\prime}$-(benzyl)ammoniumpropyl)carbomoylmethoxy]-2,8,14,20tetrathiacalix[4]arene tetrabromide (1,3-alternate 33). Yield: $0.12 \mathrm{~g}(90 \%)$, mp $131{ }^{\circ} \mathrm{C} .{ }^{1} \mathrm{H}$ NMR (400 MHz, $298 \mathrm{~K}$, DMSO-d 6 ) $\delta$ : $1.19\left(\mathrm{~s}, 36 \mathrm{H},\left(\mathrm{CH}_{3}\right)_{3} \mathrm{C}\right), 1.39\left(\mathrm{t},{ }^{3} J_{\mathrm{HH}}=7.1 \mathrm{~Hz}, 24 \mathrm{H}, \mathrm{CH}_{3} \mathrm{CH}_{2}-\right)$, $3.16\left(\mathrm{~m}, 8 \mathrm{H}, \mathrm{NCH}_{2} \mathrm{CH}_{2} \mathrm{NH}\right), 3.25\left(\mathrm{q},{ }^{3} \mathrm{~J}_{\mathrm{HH}}=7.1 \mathrm{~Hz}, 16 \mathrm{H}\right.$, $-\mathrm{CH}_{2} \mathrm{CH}_{3}$ ), $3.62\left(\mathrm{~m}, 8 \mathrm{H}, \mathrm{NCH}_{2} \mathrm{CH}_{2} \mathrm{NH}\right), 4.20$ (s, 8H, $\mathrm{OCH}_{2} \mathrm{CO}$ ), 4.60 (s, 8H, N $\left.{ }^{+} \mathrm{CH}_{2} \mathrm{Ph}\right), 7.53\left(\mathrm{~m}, 8 \mathrm{H}, \mathrm{Ar}^{\prime} \mathrm{H}\right), 7.58\left(\mathrm{~m}, 12 \mathrm{H}, \mathrm{Ar}^{\prime} \mathrm{H}\right)$, 7.60 (s, 8H, ArH), 8.38 (br. s, $4 \mathrm{H}, \mathrm{CONH}) .{ }^{13} \mathrm{C}$ NMR $(100 \mathrm{MHz}$, $298 \mathrm{~K}, \mathrm{DMSO}_{6}$ ) $\delta: 168.07,157.35,145.80,133.44,132.63$, 130.46, 129.15, 127.49, 127.18, 70.84, 60.55, 54.14, 53.19, 33.87, 32.07, 30.89, 7.57. El. anal. calcd for $\mathrm{C}_{100} \mathrm{H}_{140} \mathrm{Br}_{4} \mathrm{~N}_{8} \mathrm{O}_{8} \mathrm{~S}_{4}$ : C $59.16 \%, \mathrm{H} 6.95 \%, \mathrm{~N} 5.52 \%$, S 6.32\%. Found: C 58.95\%, H $7.05 \%$, N 5.28\%, S 6.13\%. MS (ESI): calcd for $\left[\mathrm{M}-4 \mathrm{Br}^{-}\right]^{4+} \mathrm{m} / \mathrm{z}=$ 427.6, found $m / z=427.3$. IR $\nu_{\text {max }}: 1669(\mathrm{C}=\mathrm{O}), 2954,3194(\mathrm{NH})$.

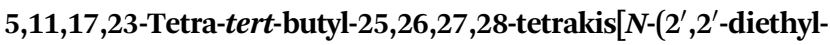
$2^{\prime}$-(ethoxycarbonylmethyl)ammoniumethyl)carbomoylmethoxy]2,8,14,20-tetrathiacalix[4] arene tetrabromide (1,3-alternate 34). Yield: $0.12 \mathrm{~g}$ (90\%), mp $115{ }^{\circ} \mathrm{C}$. ${ }^{1} \mathrm{H}$ NMR (400 MHz, $298 \mathrm{~K}$, DMSO-d $\left._{6}\right) \delta: 1.22\left(\mathrm{~s}, 36 \mathrm{H},\left(\mathrm{CH}_{3}\right)_{3} \mathrm{C}\right), 1.26\left(\mathrm{t},{ }^{3} \mathrm{~J}_{\mathrm{HH}}=7.1 \mathrm{~Hz}, 12 \mathrm{H}\right.$, $\left.\mathrm{OCH}_{2} \mathrm{CH}_{3}\right), 1.28\left(\mathrm{t},{ }^{3} J_{\mathrm{HH}}=7.0 \mathrm{~Hz}, 24 \mathrm{H}, \mathrm{CH}_{3} \mathrm{CH}_{2}-\right), 3.45-3.65(\mathrm{~m}$, $\left.32 \mathrm{H},-\mathrm{CH}_{2} \mathrm{CH}_{3},-\mathrm{NCH}_{2} \mathrm{CH}_{2} \mathrm{NH},-\mathrm{NCH}_{2} \mathrm{CH}_{2} \mathrm{NH}\right), 4.04(\mathrm{~s}, 8 \mathrm{H}$, $\mathrm{OCH}_{2} \mathrm{CO}$ ), $4.24\left(\mathrm{q},{ }^{3} \mathrm{~J}_{\mathrm{HH}}=7.1 \mathrm{~Hz}, 8 \mathrm{H}, \mathrm{OCH}_{2} \mathrm{CH}_{3}\right), 4.48(\mathrm{~s}, 8 \mathrm{H}$, $\mathrm{N}^{+} \mathrm{CH}_{2} \mathrm{CO}$ ), 7.60 (s, 8H, ArH), $8.27\left(\mathrm{t},{ }^{3} J_{\mathrm{HH}}=4.9 \mathrm{~Hz}, 4 \mathrm{H}, \mathrm{CONH}\right)$. ${ }^{13} \mathrm{C}$ NMR (100 MHz, $298 \mathrm{~K}$, DMSO-d 6 ) $\delta: 168.03,164.24,156.99$, 145.96, 133.08, 127.38, 70.57, 62.13, 55.83, 55.54, 55.08, 33.86, $32.00,30.87,13.76,7.41$. El. anal. calcd for $\mathrm{C}_{88} \mathrm{H}_{140} \mathrm{Br}_{4} \mathrm{~N}_{8} \mathrm{O}_{16} \mathrm{~S}_{4}$ : C $52.48 \% \mathrm{H} 6.53 \%, \mathrm{~N} 5.86 \%$, S $6.70 \%$. Found: C $52.64 \%, \mathrm{H}$ $6.73 \%$, N 6.06\%, S 6.33\%. MS (ESI): calcd for $\left[\mathrm{M}-4 \mathrm{Br}^{-}\right]^{4+} \mathrm{m} / z=$ 423.2, $\left[\mathrm{M}-3 \mathrm{Br}^{-}\right]^{3+} m / z=591.4,\left[\mathrm{M}-2 \mathrm{Br}^{-}\right]^{2+} m / z=927.0$, found $m / z=423.5,591.5$, 926.8. IR $\nu_{\max }$ : 1669, $1741(\mathrm{C}=\mathrm{O})$, 2957, $3187(\mathrm{NH})$.
$5,11,17,23-T e t r a-t e r t-b u t y l-25,26,27,28$-tetrakis $\left[N\right.$ - $\left(2^{\prime}, 2^{\prime}\right.$-diethyl2 '-(pentoxycarbonylmethyl)ammoniumethyl)carbomoylmethoxy]2,8,14,20-tetrathiacalix[4]arene tetrabromide (1,3-alternate 35). Yield: $0.14 \mathrm{~g}$ (94\%), mp $88{ }^{\circ} \mathrm{C}$. ${ }^{1} \mathrm{H}$ NMR (400 MHz, $298 \mathrm{~K}$, DMSO$\left.\mathrm{d}_{6}\right) \delta: 0.87\left(\mathrm{t},{ }^{3} \mathrm{~J}_{\mathrm{HH}}=6.6 \mathrm{~Hz}, 12 \mathrm{H},-\mathrm{O}\left(\mathrm{CH}_{2}\right)_{4} \mathrm{CH}_{3}\right), 1.21(\mathrm{~s}, 36 \mathrm{H}$, $\left.\left(\mathrm{CH}_{3}\right)_{3} \mathrm{C}\right), 1.25-1.32\left(\mathrm{~m}, 16 \mathrm{H}, \mathrm{O}\left(\mathrm{CH}_{2}\right)_{3} \mathrm{CH}_{2} \mathrm{CH}_{3}, \mathrm{O}\left(\mathrm{CH}_{2}\right)_{2} \mathrm{CH}_{2} \mathrm{C}_{2} \mathrm{H}_{5}\right)$, $1.28\left(\mathrm{t},{ }^{3} J_{\mathrm{HH}}=7.0 \mathrm{~Hz}, 24 \mathrm{H},\left(\mathrm{CH}_{3} \mathrm{CH}_{2} \mathrm{~N}^{+}-\right)\right), 1.63\left(\mathrm{~m}, 8 \mathrm{H}, \mathrm{OCH}_{2}-\right.$ $\left.\mathrm{CH}_{2} \mathrm{C}_{3} \mathrm{H}_{7}\right), 3.45-3.63\left(\mathrm{~m}, 32 \mathrm{H},-\mathrm{N}^{+} \mathrm{CH}_{2} \mathrm{CH}_{3},-\mathrm{NCH}_{2} \mathrm{CH}_{2} \mathrm{NH}\right.$, $\left.-\mathrm{NCH}_{2} \mathrm{CH}_{2} \mathrm{NH}\right), 4.08\left(\mathrm{~s}, 8 \mathrm{H}, \mathrm{OCH}_{2} \mathrm{CO}\right), 4.18\left(\mathrm{t},{ }^{3} J_{\mathrm{HH}}=6.4 \mathrm{~Hz}, 8 \mathrm{H}\right.$, $\mathrm{OCH}_{2} \mathrm{C}_{4} \mathrm{H}_{9}$ ), 4.51 (s, 8H, N $\mathrm{CH}_{2} \mathrm{CO}$ ), 7.60 (s, 8H, ArH), 8.29 (br. s, $4 \mathrm{H}, \mathrm{CONH}) .{ }^{13} \mathrm{C}$ NMR (100 MHz, $\left.298 \mathrm{~K}, \mathrm{DMSO}_{6}\right) \delta: 167.93$, 164.44, 157.02, 145.20, 133.11, 127.37, 70.58, 65.69, 55.87, 55.52, 55.05, 33.86, 31.53, 30.87, 27.43, 27.35, 21.66, 13.80, 7.95. El. anal. calcd for $\mathrm{C}_{100} \mathrm{H}_{164} \mathrm{Br}_{4} \mathrm{~N}_{8} \mathrm{O}_{16} \mathrm{~S}_{4}$ : C 55.04\%, H 7.57\%, N 5.13\%, S $5.88 \%$. Found: C 54.96\%, H 7.68\%, N 5.11\%, S 5.94\%. MS (ESI): calcd for $\left[\mathrm{M}-4 \mathrm{Br}^{-}\right]^{4+} \mathrm{m} / z=465.6$, found $m / z=465.6$. IR $\nu_{\max }$ : 1669, $1741(\mathrm{C}=\mathrm{O}), 2955,3300(\mathrm{NH})$.

\section{Procedure for the synthesis of compound 30}

The compound $3\left(0.10 \mathrm{~g}, 0.08 \times 10^{-3} \mathrm{~mol}\right)$ was dissolved in $10 \mathrm{ml}$ of acetonitrile in the round bottom flask equipped with magnetic stirrer and a reflux condenser. $N$-(3-Bromopropyl) phthalimide $\left(0.32 \times 10^{-3} \mathrm{~mol}\right)$ was added. The reaction mixture was refluxed for $8 \mathrm{~h}$. The solvent was removed under reduced pressure. The precipitate was dried under reduced pressure over phosphorus pentoxide.

$5,11,17,23$-Tetra-tert-butyl-25,26,27,28-tetrakis[ $N$ - $\left(3^{\prime}, 3^{\prime}\right.$-dimethyl$3^{\prime}-\left\{3^{\prime \prime}\right.$-propylphthalimide $\}$ ammoniumpropyl)carbomoylmethoxy]2,8,14,20-tetrathiacalix[4] arene tetrabromide (1,3-alternate 30). Yield: $0.14 \mathrm{~g}$ (98\%), mp $154{ }^{\circ} \mathrm{C} .{ }^{1} \mathrm{H}$ NMR (400 MHz, $298 \mathrm{~K}$, DMSO-d $\left.{ }_{6}\right) \delta: 1.20\left(\mathrm{~s}, 36 \mathrm{H},\left(\mathrm{CH}_{3}\right)_{3} \mathrm{C}\right), 1.88\left(\mathrm{~m}, 8 \mathrm{H}, \mathrm{CH}_{2} \mathrm{CH}_{2} \mathrm{CH}_{2}-\right.$ Pht), 2.06 (m, 8H, $\left.\mathrm{NHCH}_{2} \mathrm{CH}_{2} \mathrm{CH}_{2} \mathrm{~N}^{+}\right), 3.00\left(\mathrm{~s}, 24 \mathrm{H},\left(\mathrm{CH}_{3}\right)_{2} \mathrm{~N}^{+}\right)$, 3.17 (m, 8H, $\left.\mathrm{NHCH}_{2} \mathrm{CH}_{2} \mathrm{CH}_{2} \mathrm{~N}^{+}\right) 3.30$ (m, 8H, $\mathrm{CH}_{2} \mathrm{CH}_{2} \mathrm{CH}_{2} \mathrm{Pht}$ ), $3.56\left(\mathrm{~m}, 8 \mathrm{H}, \mathrm{NHCH}_{2} \mathrm{CH}_{2} \mathrm{CH}_{2} \mathrm{~N}^{+}\right), 3.70$ (m, 8H, $\mathrm{CH}_{2} \mathrm{CH}_{2} \mathrm{CH}_{2} \mathrm{Pht}$ ), 3.99 (s, 8H, $\mathrm{OCH}_{2} \mathrm{CO}$ ), 7.60 (s, 8H, ArH), 7.82-7.89 (m, 16H, Pht), $8.05\left(\mathrm{t},{ }^{3} J_{\mathrm{HH}}=5.3 \mathrm{~Hz}, 4 \mathrm{H}, \mathrm{NHCH}_{2} \mathrm{CH}_{2} \mathrm{CH}_{2} \mathrm{~N}^{+}\right) .{ }^{13} \mathrm{C} \mathrm{NMR}(100$ MHz, 298 K, DMSO-d $\left.{ }_{6}\right) \delta: 167.94,167.21,146.00,134.42,134.15$, 131.66, 127.64, 123.10, 122.96, 58.56, 49.88, 36.15, 34.69, 33.85, $31.89,31.10,30.55,21.60,20.73$. El. anal. calcd for $\mathrm{C}_{112} \mathrm{H}_{144}$ $\mathrm{Br}_{4} \mathrm{~N}_{12} \mathrm{O}_{16} \mathrm{~S}_{4}$ : C 56.94\%, H 6.14\%, N 7.12\%, S 5.43\%, Br 13.53\%. Found: C 56.91\%, H 5.85\%, N 6.04\%, S 5.46\%, Br 13.65\%. MS (ESI): calcd for $\left[\mathrm{M}-4 \mathrm{Br}^{-}\right]^{4+} m / z=510.7$, found $m / z=510.6$. IR $\nu_{\text {max }}$ : 1667, $1705(\mathrm{C}=\mathrm{O}), 2956,3315(\mathrm{~N}-\mathrm{H})$.

\section{General procedure for the synthesis of compounds 39-72}

The compounds 5-38 (0.10 g) were dissolved in $2 \mathrm{ml}$ of water in the round bottom flask equipped with magnetic stirrer. Lithium bis(trifluoromethylsulfonyl)imide was added in the molar ratio of $1: 1$ taken for each ammonium group. The reaction mixture was stirred for $24 \mathrm{~h}$. The precipitate was filtered and dried under reduced pressure over phosphorus pentoxide.

$5,11,17,23$-Tetra-tert-butyl-25,26,27,28-tetrakis $\left[\left(N-\left(3^{\prime}, 3^{\prime}, 3^{\prime}\right.\right.\right.$ trimethyl)ammoniumpropyl)carbomoylmethoxy]-2,8,14,20tetrathiacalix[4]arene tetra[bis(trifluoromethylsulfonyl)imide] (cone 39). Yield: $0.09 \mathrm{~g}$ (91\%), mp $87{ }^{\circ} \mathrm{C} .{ }^{1} \mathrm{H}$ NMR (400 MHz, 298 $\mathrm{K}$, DMSO-d $\left._{6}\right) \delta: 1.08\left(\mathrm{~s}, 36 \mathrm{H},\left(\mathrm{CH}_{3}\right)_{3} \mathrm{C}\right), 1.89(\mathrm{~m}, 8 \mathrm{H}$, 
$\left.-\mathrm{NCH}_{2} \mathrm{CH}_{2} \mathrm{CH}_{2} \mathrm{NH}\right), 3.05\left(\mathrm{~s}, 36 \mathrm{H},\left(\mathrm{CH}_{3}\right)_{3} \mathrm{~N}^{+}\right), 3.25(\mathrm{~m}, 8 \mathrm{H}$, $-\mathrm{NCH}_{2} \mathrm{CH}_{2} \mathrm{CH}_{2} \mathrm{NH}$ ), 3.27 (m, 8H, $\left.\mathrm{NCH}_{2} \mathrm{CH}_{2} \mathrm{CH}_{2} \mathrm{NH}\right), 4.82(\mathrm{~s}, 8 \mathrm{H}$, $\mathrm{OCH}_{2} \mathrm{CO}$ ), 7.40 (s, 8H, ArH), $8.49\left(\mathrm{t},{ }^{3} J_{\mathrm{HH}}=5.2 \mathrm{~Hz}, 4 \mathrm{H}, \mathrm{CONH}\right)$. ${ }^{13} \mathrm{C}$ NMR (100 MHz, $298 \mathrm{~K}, \mathrm{DMSO}-\mathrm{d}_{6}$ ) $\delta:$ 168.26, 157.99, 146.80, 134.42, 128.02, 119.96 (q), 74.22, 63.40, 52.29, 35.38, 33.91, 30.69, 22.83. El. anal. calcd for $\mathrm{C}_{80} \mathrm{H}_{116} \mathrm{~F}_{24} \mathrm{~N}_{12} \mathrm{O}_{24} \mathrm{~S}_{12}$ : C 38.89\%, H $4.73 \%$, N 6.80\%, S 15.57\%. Found: C 39.03\%, H 5.02\%, N $6.53 \%, \mathrm{~S} 15.12 \%$. MS (MALDI TOF): calcd for $\left[\mathrm{M}-\mathrm{N}^{-}\left(\mathrm{SO}_{2}-\right.\right.$ $\left.\left.\mathrm{CF}_{3}\right)_{2}\right]^{+} m / z=2188.5$, found $m / z=2189.4$. IR $\nu_{\max }: 1669(\mathrm{C}=\mathrm{O})$, 2965, $3327(\mathrm{NH})$.

$5,11,17,23-T e t r a-t e r t-b u t y l-25,26,27,28$-tetrakis $\left[\left(N-\left(3^{\prime}, 3^{\prime}\right.\right.\right.$-dimethyl-3'-ethyl)ammoniumpropyl)carbomoylmethoxy]-2,8,14,20tetrathiacalix[4]arene tetra[bis(trifluoromethylsulfonyl)imide] (cone 40). Yield: $0.10 \mathrm{~g}$ (93\%), mp $71{ }^{\circ} \mathrm{C}$. ${ }^{1} \mathrm{H}$ NMR (400 MHz, 298 $\left.\mathrm{K}, \mathrm{DMSO}_{6}\right) \delta: 1.08\left(\mathrm{~s}, 36 \mathrm{H},\left(\mathrm{CH}_{3}\right)_{3} \mathrm{C}\right), 1.22\left(\mathrm{t},{ }^{3} \mathrm{~J}_{\mathrm{HH}}=7.0 \mathrm{~Hz}\right.$, $\left.12 \mathrm{H}, \mathrm{N}^{+} \mathrm{CH}_{2} \mathrm{CH}_{3}\right) 1.87\left(\mathrm{~m}, 8 \mathrm{H},-\mathrm{NCH}_{2} \mathrm{CH}_{2} \mathrm{CH}_{2} \mathrm{NH}\right), 2.97(\mathrm{~s}, 24 \mathrm{H}$, $\left.\left(\mathrm{CH}_{3}\right)_{2} \mathrm{~N}^{+}\right), 3.23\left(\mathrm{~m}, 8 \mathrm{H},-\mathrm{NCH}_{2} \mathrm{CH}_{2} \mathrm{CH}_{2} \mathrm{NH}\right), 3.29\left(\mathrm{q},{ }^{3} \mathrm{~J}_{\mathrm{HH}}=\right.$ $\left.7.2 \mathrm{~Hz}, 8 \mathrm{H}, \mathrm{N}^{+} \mathrm{CH}_{2} \mathrm{CH}_{3}\right), 3.32\left(\mathrm{~m}, 8 \mathrm{H}, \mathrm{NCH}_{2} \mathrm{CH}_{2} \mathrm{CH}_{2} \mathrm{NH}\right), 4.82(\mathrm{~s}$, $8 \mathrm{H}, \mathrm{OCH}_{2} \mathrm{CO}$ ), 7.40 (s, 8H, ArH), 8.49 (br. s, $\left.4 \mathrm{H}, \mathrm{CONH}\right) .{ }^{13} \mathrm{C}$ NMR (100 MHz, $298 \mathrm{~K}, \mathrm{DMSO}_{6}$ ) $\delta:$ 168.24, 157.94, 146.78, 134.43, 127.99, 119.67 (q), 74.17, 60.33, 58.65, 49.53, 35.39, 33.91, 30.67, 22.35, 7.74. El. anal. calcd for $\mathrm{C}_{84} \mathrm{H}_{124} \mathrm{~F}_{24} \mathrm{~N}_{12} \mathrm{O}_{24} \mathrm{~S}_{12}$ : C $39.93 \%, \mathrm{H} 4.95 \%, \mathrm{~N} 6.65 \%, \mathrm{~S} 15.23 \%$. Found: C $39.37 \%, \mathrm{H}$ $5.00 \%, \mathrm{~N} 6.76 \%, \mathrm{~S} 14.89 \%$. MS (MALDI TOF): calcd for [M $\left.\mathrm{N}^{-}\left(\mathrm{SO}_{2} \mathrm{CF}_{3}\right)_{2}\right]^{+} m / z=2244.6$, found $m / z=2244.8$. IR $\nu_{\text {max }}: 1671$ $(\mathrm{C}=\mathrm{O}), 2965,3328(\mathrm{NH})$.

$5,11,17,23$-Tetra-tert-butyl-25,26,27,28-tetrakis $\left[\left(N\right.\right.$ - $\left(3^{\prime}, 3^{\prime}\right.$-dimethyl-3'-benzyl)ammoniumpropyl)carbomoylmethoxy]-2,8,14,20tetrathiacalix[4]arene tetra[bis(trifluoromethylsulfonyl)imide] (cone 41). Yield: $0.10 \mathrm{~g}(93 \%), \mathrm{mp} 56{ }^{\circ} \mathrm{C} .{ }^{1} \mathrm{H}$ NMR (400 MHz, 298 $\left.\mathrm{K}, \mathrm{CDCl}_{3}\right) \delta: 1.10\left(\mathrm{~s}, 36 \mathrm{H},\left(\mathrm{CH}_{3}\right)_{3} \mathrm{C}\right), 2.23\left(\mathrm{~m}, 8 \mathrm{H},-\mathrm{NCH}_{2} \mathrm{CH}_{2}-\right.$ $\left.\mathrm{CH}_{2} \mathrm{NH}\right), 2.98\left(\mathrm{~s}, 24 \mathrm{H},\left(\mathrm{CH}_{3}\right)_{2} \mathrm{~N}^{+}\right), 3.50\left(\mathrm{~m}, 8 \mathrm{H},-\mathrm{NCH}_{2} \mathrm{CH}_{2} \mathrm{CH}_{2}-\right.$ $\mathrm{NH}), 3.53\left(\mathrm{~m}, 8 \mathrm{H}, \mathrm{NCH}_{2} \mathrm{CH}_{2} \mathrm{CH}_{2} \mathrm{NH}\right), 4.42\left(\mathrm{~s}, 8 \mathrm{H}, \mathrm{N}^{+} \mathrm{CH}_{2} \mathrm{CO}\right)$, 4.91 (s, 8H, $\left.\mathrm{OCH}_{2} \mathrm{CO}\right), 7.35$ (s, 8H, ArH), $7.43\left(\mathrm{~m}, 20 \mathrm{H}, \mathrm{Ar}^{\prime} \mathrm{H}\right)$, 8.29 (br. s, 4H, CONH). ${ }^{13} \mathrm{C} \mathrm{NMR}\left(100 \mathrm{MHz}, 298 \mathrm{~K}, \mathrm{CDCl}_{3}\right) \delta$ : 169.24, 157.30, 147.80, 134.97, 132.85, 131.06, 129.43, 127.87, 126.49, 119.94 (q), 74.26, 68.37, 63.07, 49.46, 36.20, 34.38, 31.04, 23.01. El. anal. calcd for $\mathrm{C}_{104} \mathrm{H}_{132} \mathrm{~F}_{24} \mathrm{~N}_{12} \mathrm{O}_{24} \mathrm{~S}_{12}$ : C $45.01 \%, \mathrm{H}$ 4.79\%, N 6.06\%, S 13.87\%. Found: C 45.30\%, H 4.69\%, N 5.84\%, $\mathrm{S} 14.04 \%$. MS (MALDI TOF): calcd for $\left[\mathrm{M}-\mathrm{N}^{-}\left(\mathrm{SO}_{2} \mathrm{CF}_{3}\right)_{2}\right]^{+} \mathrm{m} / z=$ 2492.6, found $m / z=2493.3$. IR $\nu_{\max }: 1658(\mathrm{C}=\mathrm{O}), 2961,3328$ $(\mathrm{NH})$.

5,11,17,23-Tetra-tert-butyl-25,26,27,28-tetrakis $\left[N\right.$ - $\left(3^{\prime}, 3^{\prime}\right.$-dimethyl-3'-(ethoxycarbonylmethyl)ammoniumpropyl)carbomoylmethoxy]-2,8,14,20-tetrathiacalix[4]arene tetra[bis(trifluoromethylsulfonyl)imide] (cone 42). Yield: $0.11 \mathrm{~g}(95 \%), \mathrm{mp} 45{ }^{\circ} \mathrm{C} .{ }^{1} \mathrm{H}$ NMR (400 MHz, $\left.298 \mathrm{~K}, \mathrm{CDCl}_{3}\right) \delta: 1.11\left(\mathrm{~s}, 36 \mathrm{H},\left(\mathrm{CH}_{3}\right)_{3} \mathrm{C}\right), 1.30(\mathrm{t}$, $\left.{ }^{3} J_{\mathrm{HH}}=7.1 \mathrm{~Hz}, 12 \mathrm{H}, \mathrm{OCH}_{2} \mathrm{CH}_{3}\right), 2.14\left(\mathrm{~m}, 8 \mathrm{H},-\mathrm{NCH}_{2} \mathrm{CH}_{2} \mathrm{CH}_{2}-\right.$ $\mathrm{NH}), 3.34\left(\mathrm{~s}, 24 \mathrm{H},\left(\mathrm{CH}_{3}\right)_{2} \mathrm{~N}^{+}\right), 3.47\left(\mathrm{~m}, 8 \mathrm{H},-\mathrm{NCH}_{2} \mathrm{CH}_{2} \mathrm{CH}_{2} \mathrm{NH}\right)$, $3.72\left(\mathrm{~m}, 8 \mathrm{H}, \mathrm{NCH}_{2} \mathrm{CH}_{2} \mathrm{CH}_{2} \mathrm{NH}\right), 4.23\left(\mathrm{~s}, 8 \mathrm{H}, \mathrm{N}^{+} \mathrm{CH}_{2} \mathrm{CO}\right), 4.26$ (q, $\left.{ }^{3} \mathrm{JHH}_{\mathrm{HH}}=7.0 \mathrm{~Hz}, 8 \mathrm{H}, \mathrm{N}^{+} \mathrm{CH}_{2} \mathrm{CH}_{3}\right), 4.89$ (s, 8H, $\left.\mathrm{OCH}_{2} \mathrm{CO}\right), 7.35$ (s, 8H, ArH), 8.23 (br. s, 4H, CONH). ${ }^{13} \mathrm{C}$ NMR (100 MHz, $298 \mathrm{~K}$, $\mathrm{CDCl}_{3}$ ) $\delta: 169.12,164.07,157.27,147.82,134.87,119.67$ (q), $74.26,64.50,62.95,56.57,51.42,35.93,34.28,31.05,29.81$, 22.82 , 13.71. El. anal. calcd for $\mathrm{C}_{92} \mathrm{H}_{132} \mathrm{~F}_{24} \mathrm{~N}_{12} \mathrm{O}_{32} \mathrm{~S}_{12}$ : C $40.05 \%$, $\mathrm{H} 4.82 \%$, N 6.09\%, S 13.95\%. Found: C 39.66\%, H $4.81 \%$, N $5.77 \%$, S 13.66\%. MS (MALDI TOF): calcd for [M-
$\left.\mathrm{N}^{-}\left(\mathrm{SO}_{2} \mathrm{CF}_{3}\right)_{2}\right]^{+} m / z=2478.7$, found $m / z=2480.0$. IR $\nu_{\text {max }}: 1669$, $1747(\mathrm{C}=\mathrm{O}), 2965,3328(\mathrm{NH})$.

$5,11,17,23$-Tetra-tert-butyl-25,26,27,28-tetrakis $\left[N\right.$ - $\left(3^{\prime}, 3^{\prime}\right.$-dimethyl-3'-(pentoxycarbonylmethyl)ammoniumpropyl)carbomoylmethoxy]-2,8,14,20-tetrathiacalix[4]arene tetra[bis(trifluoromethylsulfonyl)imide] (cone 43). Yield: $0.12 \mathrm{~g}$ (94\%), mp $35{ }^{\circ} \mathrm{C}$. ${ }^{1} \mathrm{H}$ NMR (400 MHz, $\left.298 \mathrm{~K}, \mathrm{DMSO}_{6}\right) \delta: 0.87\left(\mathrm{t},{ }^{3} \mathrm{~J}_{\mathrm{HH}}=6.7 \mathrm{~Hz}\right.$, $\left.12 \mathrm{H}, \mathrm{O}\left(\mathrm{CH}_{2}\right)_{4} \mathrm{CH}_{3}\right), 1.08$ (s, 36H, $\left.\left(\mathrm{CH}_{3}\right)_{3} \mathrm{C}\right), 1.24-1.36(\mathrm{~m}, 16 \mathrm{H}$, $\left.\mathrm{O}\left(\mathrm{CH}_{2}\right)_{3} \mathrm{CH}_{2} \mathrm{CH}_{3}, \mathrm{O}\left(\mathrm{CH}_{2}\right)_{2} \mathrm{CH}_{2} \mathrm{C}_{2} \mathrm{H}_{5}\right), 1.57\left(\mathrm{~m}, 8 \mathrm{H}, \mathrm{OCH}_{2} \mathrm{CH}_{2} \mathrm{C}_{3}-\right.$ $\left.\mathrm{H}_{7}\right), 1.94\left(\mathrm{~m}, 8 \mathrm{H},-\mathrm{NCH}_{2} \mathrm{CH}_{2} \mathrm{CH}_{2} \mathrm{NH}\right), 3.16\left(\mathrm{~s}, 24 \mathrm{H},\left(\mathrm{CH}_{3}\right)_{2} \mathrm{~N}^{+}\right)$, $3.49\left(\mathrm{~m}, 8 \mathrm{H},-\mathrm{NCH}_{2} \mathrm{CH}_{2} \mathrm{CH}_{2} \mathrm{NH}\right), 3.54\left(\mathrm{~m}, 8 \mathrm{H}, \mathrm{NCH}_{2} \mathrm{CH}_{2} \mathrm{CH}_{2}-\right.$ $\mathrm{NH}), 4.14\left(\mathrm{t},{ }^{3} \mathrm{~J}_{\mathrm{HH}}=6.5 \mathrm{~Hz}, 8 \mathrm{H}, \mathrm{OCH}_{2} \mathrm{C}_{4} \mathrm{H}_{9}\right), 4.42(\mathrm{~s}, 8 \mathrm{H}$, $\mathrm{N}^{+} \mathrm{CH}_{2} \mathrm{CO}$ ), 4.79 (s, 8H, $\mathrm{OCH}_{2} \mathrm{CO}$ ), 7.39 (s, 8H, ArH), 8.47 (br. s, $4 \mathrm{H}, \mathrm{CONH}) .{ }^{13} \mathrm{C}$ NMR (100 MHz, $\left.298 \mathrm{~K}, \mathrm{DMSO}-\mathrm{d}_{6}\right) \delta: 168.26$, 164.71, 158.00, 146.79, 134.42, 127.99, 119.66 (q), 74.21, 65.76, $62.35,60.58,51.11,35.22,33.89,30.64,27.43,27.34,22.49$, 21.66, 13.77. El. anal. calcd for $\mathrm{C}_{104} \mathrm{H}_{156} \mathrm{~F}_{24} \mathrm{~N}_{12} \mathrm{O}_{32} \mathrm{~S}_{12}$ : C $42.67 \%$, H 5.37\%, N 5.74\%, S 13.15\%. Found: C 42.58\%, H 5.36\%, N $5.55 \%$, S $13.00 \%$. MS (ESI): calcd for $\left[\mathrm{M}-2 \mathrm{~N}^{-}\left(\mathrm{SO}_{2} \mathrm{CF}_{3}\right)_{2}\right]^{2+} \mathrm{m} / \mathrm{z}$ $=1183.4,\left[\mathrm{M}-3 \mathrm{~N}^{-}\left(\mathrm{SO}_{2} \mathrm{CF}_{3}\right)_{2}\right]^{3+} m / z=695.0,\left[\mathrm{M}-4 \mathrm{~N}^{-}\left(\mathrm{SO}_{2}-\right.\right.$ $\left.\left.\mathrm{CF}_{3}\right)_{2}\right]^{4+} m / z=451.6$, found $m / z=1183.0,695.3$, 451.5. IR $\nu_{\max }$ : 1669, $1747(\mathrm{C}=\mathrm{O}), 2962,3329(\mathrm{NH})$.

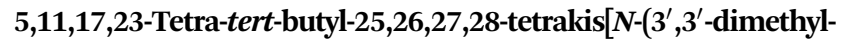
$3^{\prime}$-\{(ethoxycarbonylmethyl)amidocarbonylmethyl $\}$ ammoniumpropyl)carbomoylmethoxy]-2,8,14,20-tetrathiacalix[4]arene tetra[bis(trifluoromethylsulfonyl)imide] (cone 44). Yield: $0.13 \mathrm{~g}$ (97\%), mp $63{ }^{\circ} \mathrm{C} .{ }^{1} \mathrm{H}$ NMR (400 MHz, $\left.298 \mathrm{~K}, \mathrm{CDCl}_{3}\right) \delta: 1.11$ (s, $\left.36 \mathrm{H},\left(\mathrm{CH}_{3}\right)_{3} \mathrm{C}\right), 1.26\left(\mathrm{t},{ }^{3} J_{\mathrm{HH}}=7.1 \mathrm{~Hz}, 12 \mathrm{H}, \mathrm{CH}_{3} \mathrm{CH}_{2} \mathrm{O}-\right), 2.17(\mathrm{~m}$, $\left.8 \mathrm{H}, \mathrm{NHCH}_{2} \mathrm{CH}_{2} \mathrm{CH}_{2} \mathrm{~N}^{+}\right), 3.31\left(\mathrm{~s}, 12 \mathrm{H},\left(\mathrm{CH}_{3}\right)_{2} \mathrm{~N}^{+}\right), 3.47(\mathrm{~m}, 8 \mathrm{H}$, $\mathrm{NHCH}_{2} \mathrm{CH}_{2} \mathrm{CH}_{2} \mathrm{~N}^{+}$), $3.62\left(\mathrm{~m}, 8 \mathrm{H}, \mathrm{NHCH}_{2} \mathrm{CH}_{2} \mathrm{CH}_{2} \mathrm{~N}^{+}\right.$), 3.97 (d, $\left.{ }^{3} J_{\mathrm{HH}}=5.5 \mathrm{~Hz}, 8 \mathrm{H}, \mathrm{NHCH}_{2} \mathrm{CO}\right), 4.10\left(\mathrm{~s}, 8 \mathrm{H}, \mathrm{N}^{+} \mathrm{CH}_{2} \mathrm{CO}\right), 4.15$ (q, $\left.{ }^{3} J_{\mathrm{HH}}=7.1 \mathrm{~Hz}, 8 \mathrm{H}, \mathrm{CH}_{3} \mathrm{CH}_{2} \mathrm{O}-\right), 4.89\left(\mathrm{~s}, 8 \mathrm{H}, \mathrm{OCH}_{2} \mathrm{CO}\right), 7.35(\mathrm{~s}$, $8 \mathrm{H}, \mathrm{ArH}), 7.77\left(\mathrm{t},{ }^{3} J_{\mathrm{HH}}=5.8 \mathrm{~Hz}, 4 \mathrm{H}, \mathrm{NHCH}_{2} \mathrm{CH}_{2} \mathrm{CH}_{2} \mathrm{~N}^{+}\right), 8.23$ (br. s, 4H, $\left.\mathrm{NHCH}_{2} \mathrm{CO}\right) .{ }^{13} \mathrm{C} \mathrm{NMR}\left(100 \mathrm{MHz}, 298 \mathrm{~K}, \mathrm{CDCl}_{3}\right) \delta$ : $169.35,168.48,162.93$, 157.12, 147.89, 135.02, 127.69, 119.75 (q), 74.26, 64.84, 62.56, 61.60, 51.98, 41.34, 35.99, 34.34, 31.04, 22.96, 14.03. El. anal. calcd for $\mathrm{C}_{100} \mathrm{H}_{144} \mathrm{~F}_{24} \mathrm{~N}_{16} \mathrm{O}_{36} \mathrm{~S}_{12}$ : C 40.21\%, $\mathrm{H} 4.86 \%$, N 7.50\%, S 12.88\%. Found: C 40.12\%, H $5.12 \%, \mathrm{~N}$ $7.53 \%$, S $12.47 \%$. MS (ESI): calcd for $\left[\mathrm{M}-3 \mathrm{~N}^{-}\left(\mathrm{SO}_{2} \mathrm{CF}_{3}\right)_{2}\right]^{3+} \mathrm{m} / \mathrm{z}$ $=715.5,\left[\mathrm{M}-4 \mathrm{~N}^{-}\left(\mathrm{SO}_{2} \mathrm{CF}_{3}\right)_{2}\right]^{4+} m / z=466.6$, found $\mathrm{m} / z=715.3$, 466.6. IR $\nu_{\text {max }}$ : 1670, $1743(\mathrm{C}=\mathrm{O}), 2966,3354(\mathrm{~N}-\mathrm{H})$.

$\mathbf{5 , 1 1 , 1 7 , 2 3 - T e t r a - t e r t - b u t y l - 2 5 , 2 6 , 2 7 , 2 8 - t e t r a k i s}\left[\mathrm{N}\right.$-( $3^{\prime}, \mathbf{3}^{\prime}$-dimethyl$3^{\prime}-\{([$ ethoxycarbonylmethyl $]$ amidocarbonylmethyl)amidocarbonylmethyl $\}$ ammoniumpropyl)carbomoylmethoxy]-2,8,14,20tetrathiacalix[4]arene tetra[bis(trifluoromethylsulfonyl)imide] (cone 45). Yield: $0.14 \mathrm{~g}$ (96\%), mp $69{ }^{\circ} \mathrm{C} .{ }^{1} \mathrm{H}$ NMR $(400 \mathrm{MHz}, 298$ $\left.\mathrm{K}, \mathrm{DMSO}_{6}\right) \delta: 1.08\left(\mathrm{~s}, 36 \mathrm{H},\left(\mathrm{CH}_{3}\right)_{3} \mathrm{C}\right), 1.19\left(\mathrm{t},{ }^{3} J_{\mathrm{HH}}=7.1 \mathrm{~Hz}\right.$, $\left.12 \mathrm{H}, \mathrm{CH}_{3} \mathrm{CH}_{2} \mathrm{O}-\right), 1.93\left(\mathrm{~m}, 8 \mathrm{H}, \mathrm{NHCH}_{2} \mathrm{CH}_{2} \mathrm{CH}_{2} \mathrm{~N}^{+}\right), 3.16(\mathrm{~s}, 12 \mathrm{H}$, $\left.\left(\mathrm{CH}_{3}\right)_{2} \mathrm{~N}^{+}\right), 3.24\left(\mathrm{~m}, 8 \mathrm{H}, \mathrm{NHCH}_{2} \mathrm{CH}_{2} \mathrm{CH}_{2} \mathrm{~N}^{+}\right), 3.47(\mathrm{~m}, 8 \mathrm{H}$, $\mathrm{NHCH}_{2} \mathrm{CH}_{2} \mathrm{CH}_{2} \mathrm{~N}^{+}$), $3.86\left(\mathrm{~d},{ }^{3} J_{\mathrm{HH}}=5.7 \mathrm{~Hz}, 8 \mathrm{H}, \mathrm{NHCH}_{2} \mathrm{CO}\right), 3.87$ $\left(\mathrm{d},{ }^{3} \mathrm{~J}_{\mathrm{HH}}=6.1 \mathrm{~Hz}, 8 \mathrm{H}, \mathrm{NHCH}_{2} \mathrm{CO}\right), 4.06\left(\mathrm{~s}, 8 \mathrm{H}, \mathrm{N}^{+} \mathrm{CH}_{2} \mathrm{CO}\right), 4.09$ (q, ${ }^{3} \mathrm{~J}_{\mathrm{HH}}=7.1 \mathrm{~Hz}, 8 \mathrm{H}, \mathrm{CH}_{3} \mathrm{CH}_{2} \mathrm{O}-$ ), 4.80 (s, 8H, $\mathrm{OCH}_{2} \mathrm{CO}$ ), 7.40 (s, $8 \mathrm{H}, \mathrm{ArH}$ ), 8.50 (br. s, $16 \mathrm{H}, \mathrm{NHCH}_{2} \mathrm{CH}_{2} \mathrm{CH}_{2} \mathrm{~N}^{+}, \mathrm{NHCH}_{2} \mathrm{CO}$ ), $8.82\left(\mathrm{t},{ }^{3} J_{\mathrm{HH}}=5.9 \mathrm{~Hz}, 4 \mathrm{H}, \mathrm{CONH}\right) .{ }^{13} \mathrm{C} \mathrm{NMR}(100 \mathrm{MHz}, 298 \mathrm{~K}$, DMSO-d $_{6}$ ) $\delta: 169.75,168.51,163.04,157.72,146.88,134.43$, $127.82,119.48$ (q), 73.34, 62.73, 62.02, 60.50, 54.78, 51.23, 41.53, 40.59 , 35.36, 33.85, 30.68, 22.52, 13.97. El. anal. calcd for 
$\mathrm{C}_{108} \mathrm{H}_{156} \mathrm{~F}_{24} \mathrm{~N}_{20} \mathrm{O}_{40} \mathrm{~S}_{12}$ : C $40.34 \%, \mathrm{H} 4.89 \%$, N 8.71\%, S $11.97 \%$. Found: $\mathrm{C} 41.00 \%, \mathrm{H} 4.71 \%$, N 7.58\%, S 12.41\%. MS (ESI): calcd for $\left[\mathrm{M}-4 \mathrm{~N}^{-}\left(\mathrm{SO}_{2} \mathrm{CF}_{3}\right)_{2}\right]^{4+} \mathrm{m} / z=523.7$, found $\mathrm{m} / z=523.6$. IR $\nu_{\text {max }}: 1663(\mathrm{C}=\mathrm{O}), 2965,3342(\mathrm{~N}-\mathrm{H})$.

$5,11,17,23-T e t r a-t e r t-b u t y l-25,26,27,28-t e t r a k i s\left[N-\left(3^{\prime}, 3^{\prime}\right.\right.$-dimethyl$3^{\prime}$ - $\{($ ethoxycarbonyl $[S$-methyl $]$ methyl)amidocarbonylmethyl $\}$ ammoniumpropyl)carbomoylmethoxy]-2,8,14,20-tetrathiacalix [4] arene tetra[bis(trifluoromethylsulfonyl)imide] (cone 46). Yield: $0.12 \mathrm{~g}$ (90\%), mp $56{ }^{\circ} \mathrm{C} .{ }^{1} \mathrm{H}$ NMR (400 MHz, $298 \mathrm{~K}$, DMSO$\left.\mathrm{d}_{6}\right) \delta: 1.08\left(\mathrm{~s}, 36 \mathrm{H},\left(\mathrm{CH}_{3}\right)_{3} \mathrm{C}\right), 1.19\left(\mathrm{t},{ }^{3} J_{\mathrm{HH}}=7.1 \mathrm{~Hz}, 12 \mathrm{H}\right.$, $\mathrm{CH}_{3} \mathrm{CH}_{2} \mathrm{O}-$ ), 1.31 (d, $\left.{ }^{3} J_{\mathrm{HH}}=7.3 \mathrm{~Hz}, 12 \mathrm{H}, \mathrm{CH}_{3} \mathrm{CH}\right), 1.92(\mathrm{~m}, 8 \mathrm{H}$, $\left.\mathrm{NHCH}_{2} \mathrm{CH}_{2} \mathrm{CH}_{2} \mathrm{~N}^{+}\right), 3.16\left(\mathrm{~s}, 12 \mathrm{H},\left(\mathrm{CH}_{3}\right)_{2} \mathrm{~N}^{+}\right), 3.23(\mathrm{~m}, 8 \mathrm{H}$, $\left.\mathrm{NHCH}_{2} \mathrm{CH}_{2} \mathrm{CH}_{2} \mathrm{~N}^{+}\right), 3.48\left(\mathrm{~m}, 8 \mathrm{H}, \mathrm{NHCH}_{2} \mathrm{CH}_{2} \mathrm{CH}_{2} \mathrm{~N}^{+}\right), 4.06(\mathrm{~s}$, $8 \mathrm{H}, \mathrm{N}^{+} \mathrm{CH}_{2} \mathrm{CO}$ ), 4.09 (m, 8H, $\left.\mathrm{CH}_{3} \mathrm{CH}_{2} \mathrm{O}-\right)$, 4.26 (m, $4 \mathrm{H}, \mathrm{CH}_{3} \mathrm{CH}$ ), $4.80\left(\mathrm{~s}, 8 \mathrm{H}, \mathrm{OCH}_{2} \mathrm{CO}\right), 7.39(\mathrm{~s}, 8 \mathrm{H}, \mathrm{ArH}), 8.50\left(\mathrm{t},{ }^{3} J_{\mathrm{HH}}=5.4 \mathrm{~Hz}\right.$, $\left.4 \mathrm{H}, \mathrm{NHCH}_{2} \mathrm{CH}_{2} \mathrm{CH}_{2} \mathrm{~N}^{+}\right), 9.02\left(\mathrm{~d},{ }^{3} J_{\mathrm{HH}}=6.7 \mathrm{~Hz}, 4 \mathrm{H}, \mathrm{CONH}\right) .{ }^{13} \mathrm{C}$ NMR (100 MHz, $298 \mathrm{~K}, \mathrm{DMSO}_{6}$ ) $\delta:$ 171.45, 167.98, 163.20, 158.28, 146.99, 134.03, 127.79, 119.49 (q), 74.03, 62.43, 61.69, 60.83, 51.16, 47.75, 35.12, 33.52, 30.36, 22.74, 16.63, 13.51. El. anal. calcd for $\mathrm{C}_{104} \mathrm{H}_{152} \mathrm{~F}_{24} \mathrm{~N}_{16} \mathrm{O}_{36} \mathrm{~S}_{12}$ : C $41.05 \%, \mathrm{H} 5.03 \%, \mathrm{~N}$ $7.36 \%$, S 12.64\%. Found: C $40.51 \%, \mathrm{H} 4.69 \%, \mathrm{~N} 6.16 \%, \mathrm{~S}$ $13.32 \%$. MS (ESI): calcd for $\left[\mathrm{M}-4 \mathrm{~N}^{-}\left(\mathrm{SO}_{2} \mathrm{CF}_{3}\right)_{2}\right]^{4+} \mathrm{m} / z=480.6$, found $m / z=480.5$. IR $\nu_{\max }: 1672(\mathrm{C}=\mathrm{O}), 2966,3353(\mathrm{~N}-\mathrm{H})$.

$5,11,17,23$-Tetra-tert-butyl-25,26,27,28-tetrakis $\left[N-\left(3^{\prime}, 3^{\prime}\right.\right.$-dimethyl$3^{\prime}-\left\{3^{\prime \prime}\right.$-propylphthalimide $\}$ ammoniumpropyl)carbomoylmethoxy $]$ 2,8,14,20-tetrathiacalix[4] arene tetra[bis(trifluoromethylsulfonyl)imide] (cone 47). Yield: $0.13 \mathrm{~g}$ (93\%), mp $83{ }^{\circ} \mathrm{C} .{ }^{1} \mathrm{H} \mathrm{NMR}$ $\left(400 \mathrm{MHz}, 298 \mathrm{~K}, \mathrm{DMSO}_{6}\right) \delta: 1.07\left(\mathrm{~s}, 36 \mathrm{H},\left(\mathrm{CH}_{3}\right)_{3} \mathrm{C}\right), 1.88(\mathrm{~m}$, $8 \mathrm{H}, \mathrm{CH}_{2} \mathrm{CH}_{2} \mathrm{CH}_{2} \mathrm{Pht}$ ), 2.05 (m, 8H, $\mathrm{NHCH}_{2} \mathrm{CH}_{2} \mathrm{CH}_{2} \mathrm{~N}^{+}$), 2.98 (s, $\left.24 \mathrm{H},\left(\mathrm{CH}_{3}\right)_{2} \mathrm{~N}^{+}\right), 3.22-3.30\left(\mathrm{~m}, 16 \mathrm{H}, \mathrm{NHCH}_{2} \mathrm{CH}_{2} \mathrm{CH}_{2} \mathrm{~N}, \mathrm{CH}_{2}-\right.$ $\mathrm{CH}_{2} \mathrm{CH}_{2} \mathrm{Pht}$ ), $3.37\left(\mathrm{~m}, 8 \mathrm{H}, \mathrm{NHCH}_{2} \mathrm{CH}_{2} \mathrm{CH}_{2} \mathrm{~N}^{+}\right), 3.67\left(\mathrm{t},{ }^{3} J_{\mathrm{HH}}=\right.$ $\left.5.9 \mathrm{~Hz}, 8 \mathrm{H}, \mathrm{CH}_{2} \mathrm{CH}_{2} \mathrm{CH}_{2} \mathrm{Pht}\right), 4.80$ (s, 8H, $\left.\mathrm{OCH}_{2} \mathrm{CO}\right), 7.38$ (s, 8H, ArH), $7.80-7.88(\mathrm{~m}, 16 \mathrm{H}, \mathrm{Pht}), 8.50\left(\mathrm{t},{ }^{3} J_{\mathrm{HH}}=5.2 \mathrm{~Hz}, 4 \mathrm{H}\right.$, $\left.\mathrm{NHCH}_{2} \mathrm{CH}_{2} \mathrm{CH}_{2} \mathrm{~N}^{+}\right) .{ }^{13} \mathrm{C}$ NMR (100 MHz, $298 \mathrm{~K}$, DMSO-d 6 ) $\delta$ : 168.22, 168.01, 158.00, 146.77, 134.42, 131.65, 128.03, 123.03, 119.52 (q), 74.24, 61.22, 60.96, 50.07, 35.37, 34.58, 33.91, 30.68, $22.44,21.64$. El. anal. calcd for $\mathrm{C}_{120} \mathrm{H}_{144} \mathrm{~F}_{24} \mathrm{~N}_{16} \mathrm{O}_{32} \mathrm{~S}_{12}$ : C $45.56 \%$, $\mathrm{H}$ 4.59\%, N 7.08\%, S 12.16\%. Found: C 46.16\%, H $4.45 \%, \mathrm{~N}$ $6.61 \%$, S 12.79\%. MS (ESI): calcd for $\left[\mathrm{M}-4 \mathrm{~N}^{-}\left(\mathrm{SO}_{2} \mathrm{CF}_{3}\right)_{2}\right]^{4+} \mathrm{m} / \mathrm{z}$ $=480.6$, found $m / z=480.6$. IR $\nu_{\max }: 1668,1708,1772(\mathrm{C}=\mathrm{O})$, 2963, $3334(\mathrm{~N}-\mathrm{H})$.

$5,11,17,23-T e t r a-t e r t-b u t y l-25,26,27,28-t e t r a k i s\left[\left(N-\left(2^{\prime}\right.\right.\right.$-methyl$2^{\prime}, 2^{\prime}$-diethyl)ammoniumethyl)carbomoylmethoxy]-2,8,14,20tetrathiacalix[4]arene tetra[bis(trifluoromethylsulfonyl)imide] (cone 48). Yield: $0.12 \mathrm{~g}$ (90\%), mp $72{ }^{\circ} \mathrm{C} .{ }^{1} \mathrm{H}$ NMR (400 MHz, 298 $\left.\mathrm{K}, \mathrm{CDCl}_{3}\right) \delta: 1.11\left(\mathrm{~s}, 36 \mathrm{H},\left(\mathrm{CH}_{3}\right)_{3} \mathrm{C}\right), 1.32\left(\mathrm{t},{ }^{3} J_{\mathrm{HH}}=7.2 \mathrm{~Hz}, 24 \mathrm{H}\right.$, $\left.\mathrm{CH}_{3} \mathrm{CH}_{2}-\right), 3.03\left(\mathrm{~s}, 12 \mathrm{H},\left(\mathrm{CH}_{3}\right) \mathrm{N}^{+}\right), 3.38\left(\mathrm{q},{ }^{3} J_{\mathrm{HH}}=7.2 \mathrm{~Hz}, 16 \mathrm{H}\right.$, $\left.-\mathrm{CH}_{2} \mathrm{CH}_{3}\right), 3.48\left(\mathrm{t},{ }^{3} J_{\mathrm{HH}}=6.3 \mathrm{~Hz}, 8 \mathrm{H},-\mathrm{NCH}_{2} \mathrm{CH}_{2} \mathrm{NH}\right), 3.85(\mathrm{~m}$, $8 \mathrm{H}, \mathrm{NCH}_{2} \mathrm{CH}_{2} \mathrm{NH}$ ), 4.91 (s, 8H, $\mathrm{OCH}_{2} \mathrm{CO}$ ), 7.36 (s, 8H, ArH), 8.36 (br. s, 8H, CONH). ${ }^{13} \mathrm{C}$ NMR $\left(100 \mathrm{MHz}, 298 \mathrm{~K}, \mathrm{CDCl}_{3}\right) \delta: 169.70$, 156.74, 148.21, 135.01, 127.79, 119.72 (q), 73.88, 59.11, 56.99, 47.52, 34.35, 32.94, 31.02, 7.62. El. anal. calcd for $\mathrm{C}_{84} \mathrm{H}_{124} \mathrm{~F}_{24} \mathrm{~N}_{12} \mathrm{O}_{24} \mathrm{~S}_{12}$ : C 39.93\%, H 4.95\%, N 6.65\%, S $15.23 \%$. Found: C 40.05\%, H 5.06\%, N 6.78\%, S 15.02\%. MS (ESI): calcd for $\left[\mathrm{M}-3 \mathrm{~N}^{-}\left(\mathrm{SO}_{2} \mathrm{CF}_{3}\right)_{2}\right]^{3+} \mathrm{m} / z=562.0$, found $m / z=561.9$. IR $\nu_{\text {max }}: 1667(\mathrm{C}=\mathrm{O}), 2965,3315(\mathrm{NH})$.
$5,11,17,23-T e t r a-t e r t-b u t y l-25,26,27,28$-tetrakis $\left[\left(N-\left(2^{\prime}, 2^{\prime}, 2^{\prime}-\right.\right.\right.$ triethyl)ammoniumethyl)carbomoylmethoxy]-2,8,14,20-tetrathiacalix[4] arene tetra[bis(trifluoromethylsulfonyl)imide] (cone 49). Yield: $0.12 \mathrm{~g}$ (95\%), mp $68{ }^{\circ} \mathrm{C} .{ }^{1} \mathrm{H}$ NMR (400 MHz, $298 \mathrm{~K}$, $\left.\mathrm{CDCl}_{3}\right) \delta: 1.11\left(\mathrm{~s}, 36 \mathrm{H},\left(\mathrm{CH}_{3}\right)_{3} \mathrm{C}\right), 1.30\left(\mathrm{t},{ }^{3} J_{\mathrm{HH}}=7.1 \mathrm{~Hz}, 36 \mathrm{H}\right.$, $\left.\mathrm{CH}_{3} \mathrm{CH}_{2}-\right), 3.33\left(\mathrm{q},{ }^{3} J_{\mathrm{HH}}=7.1 \mathrm{~Hz}, 24 \mathrm{H},-\mathrm{CH}_{2} \mathrm{CH}_{3}\right), 3.40(\mathrm{~m}, 8 \mathrm{H}$, $\left.-\mathrm{NCH}_{2} \mathrm{CH}_{2} \mathrm{NH}\right), 3.77\left(\mathrm{~m}, 8 \mathrm{H}, \mathrm{NCH}_{2} \mathrm{CH}_{2} \mathrm{NH}\right), 4.91(\mathrm{~s}, 8 \mathrm{H}$, $\mathrm{OCH}_{2} \mathrm{CO}$ ), 7.36 (s, 8H, ArH), 8.40 (br. s, 8H, CONH). ${ }^{13} \mathrm{C} \mathrm{NMR}$ $\left(100 \mathrm{MHz}, 298 \mathrm{~K}, \mathrm{CDCl}_{3}\right) \delta: 169.75,156.70,148.16,135.01$, $127.83,119.75$ (q), 73.85, 55.03, 53.51, 34.34, 32.66, 31.02, 7.28. El. anal. calcd for $\mathrm{C}_{88} \mathrm{H}_{132} \mathrm{~F}_{24} \mathrm{~N}_{12} \mathrm{O}_{24} \mathrm{~S}_{12}$ : C $40.92 \%, \mathrm{H} 5.15 \%, \mathrm{~N}$ $6.51 \%$, S 14.90\%. Found: C $41.11 \%$, H $5.13 \%, \mathrm{~N} 6.58 \%, \mathrm{~S}$ 14.67\%. MS (ESI): calcd for $\left[\mathrm{M}-4 \mathrm{~N}^{-}\left(\mathrm{SO}_{2} \mathrm{CF}_{3}\right)_{2}\right]^{4+} \mathrm{m} / z=365.6$, $\left[\mathrm{M}-3 \mathrm{~N}^{-}\left(\mathrm{SO}_{2} \mathrm{CF}_{3}\right)_{2}\right]^{3+} m / z=580.7$, found $m / z=365.7,580.5$. IR $\nu_{\text {max }}: 1674(\mathrm{C}=\mathrm{O}), 2963,3314(\mathrm{NH})$.

$5,11,17,23-T e t r a-t e r t-b u t y l-25,26,27,28-t e t r a k i s\left[\left(N-\left(2^{\prime}, 2^{\prime}\right.\right.\right.$-diethyl$2^{\prime}$-benzyl)ammoniumethyl)carbomoylmethoxy]-2,8,14,20-tetrathiacalix[4] arene tetra[bis(trifluoromethylsulfonyl)imide] (cone 50). Yield: $0.12 \mathrm{~g}(94 \%), \mathrm{mp} 60{ }^{\circ} \mathrm{C} .{ }^{1} \mathrm{H}$ NMR $(400 \mathrm{MHz}, 298 \mathrm{~K}$, $\left.\mathrm{CDCl}_{3}\right) \delta: 1.10\left(\mathrm{~s}, 36 \mathrm{H},\left(\mathrm{CH}_{3}\right)_{3} \mathrm{C}\right), 1.39\left(\mathrm{t},{ }^{3} J_{\mathrm{HH}}=7.1 \mathrm{~Hz}, 24 \mathrm{H}\right.$, $\left.\left(\mathrm{CH}_{3} \mathrm{CH}_{2}-\right)\right), 3.24\left(\mathrm{q},{ }^{3} \mathrm{~J}_{\mathrm{HH}}=6.9 \mathrm{~Hz}, 16 \mathrm{H},-\mathrm{CH}_{2} \mathrm{CH}_{3}\right), 3.39\left(\mathrm{t},{ }^{3} J_{\mathrm{HH}}\right.$ $\left.=7.1 \mathrm{~Hz}, 8 \mathrm{H},-\mathrm{NCH}_{2} \mathrm{CH}_{2} \mathrm{NH}\right), 3.93\left(\mathrm{~m}, 8 \mathrm{H}, \mathrm{NCH}_{2} \mathrm{CH}_{2} \mathrm{NH}\right), 4.43$ (s, 8H, N $\left.\mathrm{CH}_{2} \mathrm{Ph}\right), 4.95$ (s, 8H, $\left.\mathrm{OCH}_{2} \mathrm{CO}\right), 7.35$ (s, 8H, ArH), 7.41-7.46 (m, 20H, Ar' H). 8.51 (br. s, 4H, CONH). ${ }^{13} \mathrm{C}$ NMR (100 $\left.\mathrm{MHz}, 298 \mathrm{~K}, \mathrm{CDCl}_{3}\right) \delta: 169.90,156.78,148.04,135.0,132.36$, 131.06, 129.62, 127.82, 126.19, 119.74 (q), 73.85, 61.76, 55.18, 53.70, 34.18, 32.78, 31.02, 7.83. El. anal. calcd for $\mathrm{C}_{108} \mathrm{H}_{140} \mathrm{~F}_{24} \mathrm{~N}_{12} \mathrm{O}_{24} \mathrm{~S}_{12}$ : C $45.82 \%$, H 4.98\%, N 5.94\%, S $13.59 \%$. Found: C 45.86\%, H 5.23\%, N 6.12\%, S 13.44\%. MS (ESI): calcd for $\left[\mathrm{M}-4 \mathrm{~N}^{-}\left(\mathrm{SO}_{2} \mathrm{CF}_{3}\right)_{2}\right]^{4+} \mathrm{m} / z=427.6$, found $\mathrm{m} / z=427.4$. IR $\nu_{\text {max }}: 1672(\mathrm{C}=\mathrm{O}), 2967,3330(\mathrm{NH})$.

$5,11,17,23-T e t r a-t e r t-b u t y l-25,26,27,28$-tetrakis $\left[N-\left(2^{\prime}, 2^{\prime}\right.\right.$-diethyl2 '-(ethoxycarbonylmethyl)ammoniumethyl)carbomoylmethoxy]-

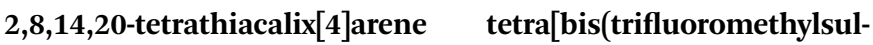
fonyl)imide] (cone 51). Yield: $0.12 \mathrm{~g}$ (92\%), mp $43{ }^{\circ} \mathrm{C} .{ }^{1} \mathrm{H} \mathrm{NMR}$ $\left(400 \mathrm{MHz}, 298 \mathrm{~K}, \mathrm{CDCl}_{3}\right) \delta: 1.11\left(\mathrm{~s}, 36 \mathrm{H},\left(\mathrm{CH}_{3}\right)_{3} \mathrm{C}\right), 1.25(\mathrm{~m}, 12 \mathrm{H}$, $\left.\mathrm{OCH}_{2} \mathrm{CH}_{3}\right), 1.33\left(\mathrm{t},{ }^{3} \mathrm{JHH}_{\mathrm{HH}}=7.2 \mathrm{~Hz}, 24 \mathrm{H}, \mathrm{CH}_{3} \mathrm{CH}_{2}-\right), 3.66(\mathrm{~m}, 16 \mathrm{H}$, $\left.-\mathrm{CH}_{2} \mathrm{CH}_{3}\right), 3.73\left(\mathrm{~m}, 8 \mathrm{H},-\mathrm{NCH}_{2} \mathrm{CH}_{2} \mathrm{NH}\right), 3.83\left(\mathrm{~m}, 8 \mathrm{H}, \mathrm{NCH}_{2}-\right.$ $\left.\mathrm{CH}_{2} \mathrm{NH}\right), 4.14\left(\mathrm{~m}, 8 \mathrm{H}, \mathrm{OCH}_{2} \mathrm{CH}_{3}\right), 4.19$ (s, 8H, N $\left.{ }^{+} \mathrm{CH}_{2} \mathrm{CO}\right), 4.90$ (s, 8H, $\mathrm{OCH}_{2} \mathrm{CO}$ ), 7.36 (s, 8H, ArH), 8.36 (br. s, $\left.4 \mathrm{H}, \mathrm{CONH}\right) .{ }^{13} \mathrm{C}$ NMR (100 MHz, $298 \mathrm{~K}, \mathrm{CDCl}_{3}$ ) $\delta: 169.90,163.93,156.72,148.19$, 142.94, 139.0, 135.06, 127.73, 119.89 (q), 73.89, 62.97, 56.99, 55.74, 34.40, 32.92, 31.02, 13.68, 7.60. El. anal. calcd for $\mathrm{C}_{96} \mathrm{H}_{140} \mathrm{~F}_{24} \mathrm{~N}_{12} \mathrm{O}_{32} \mathrm{~S}_{12}$ : C $40.96 \%$, H 5.01\%, N 5.97\%, S $13.67 \%$. Found: C $41.11 \%$, H $4.89 \%$, N 6.01\%, S 13.45\%. MS (ESI): calcd for $\left[\mathrm{M}-2 \mathrm{~N}^{-}\left(\mathrm{SO}_{2} \mathrm{CF}_{3}\right)_{2}\right]^{2+} m / z=1127.3,\left[\mathrm{M}-3 \mathrm{~N}^{-}\left(\mathrm{SO}_{2} \mathrm{CF}_{3}\right)_{2}\right]^{3+}$ $m / z=657.6,\left[\mathrm{M}-4 \mathrm{~N}^{-}\left(\mathrm{SO}_{2} \mathrm{CF}_{3}\right)_{2}\right]^{4+} \mathrm{m} / z=423.6$, found $m / z=$ 1126.9, 657.6, 423.5. IR $\nu_{\text {max }}$ : 1669, $1748(\mathrm{C}=\mathrm{O}), 2962,3328$ $(\mathrm{NH})$.

$5,11,17,23-T e t r a-t e r t-b u t y l-25,26,27,28-t e t r a k i s\left[N-\left(2^{\prime}, 2^{\prime}\right.\right.$-diethyl2 -(pentoxycarbonylmethyl)ammoniumethyl)carbomoylmethoxy]-

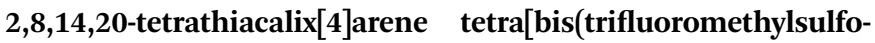
nyl)imide] (cone 52). Yield: $0.13 \mathrm{~g}$ (93\%), mp $39{ }^{\circ} \mathrm{C} .{ }^{1} \mathrm{H}$ NMR $\left(400 \mathrm{MHz}, 298 \mathrm{~K}, \mathrm{DMSO}_{6}\right) \delta: 0.86\left(\mathrm{t},{ }^{3} \mathrm{~J}_{\mathrm{HH}}=6.8 \mathrm{~Hz}, 12 \mathrm{H}\right.$, $\left.\mathrm{O}\left(\mathrm{CH}_{2}\right)_{4} \mathrm{CH}_{3}\right), 1.08\left(\mathrm{~s}, 36 \mathrm{H},\left(\mathrm{CH}_{3}\right)_{3} \mathrm{C}\right), 1.21-1.32(\mathrm{~m}, 16 \mathrm{H}$, $\left.\mathrm{O}\left(\mathrm{CH}_{2}\right)_{3} \mathrm{CH}_{2} \mathrm{CH}_{3}, \mathrm{O}\left(\mathrm{CH}_{2}\right)_{2} \mathrm{CH}_{2} \mathrm{C}_{2} \mathrm{H}_{5}\right), 1.26\left(\mathrm{t},{ }^{3} J_{\mathrm{HH}}=7.1 \mathrm{~Hz}, 24 \mathrm{H}\right.$, 
$\left.\mathrm{CH}_{3} \mathrm{CH}_{2} \mathrm{~N}^{+}\right), 1.57\left(\mathrm{~m}, 8 \mathrm{H}, \mathrm{OCH}_{2} \mathrm{CH}_{2} \mathrm{C}_{3} \mathrm{H}_{7}\right), 3.52-3.60(\mathrm{~m}, 32 \mathrm{H}$, $\left.-\mathrm{N}^{+} \mathrm{CH}_{2} \mathrm{CH}_{3},-\mathrm{NCH}_{2} \mathrm{CH}_{2} \mathrm{NH},-\mathrm{NCH}_{2} \mathrm{CH}_{2} \mathrm{NH}\right), 4.10\left(\mathrm{t},{ }^{3} \mathrm{~J}_{\mathrm{HH}}=\right.$ $\left.6.8 \mathrm{~Hz}, 8 \mathrm{H}, \mathrm{OCH}_{2} \mathrm{C}_{4} \mathrm{H}_{9}\right), 4.44\left(\mathrm{~s}, 8 \mathrm{H}, \mathrm{N}^{+} \mathrm{CH}_{2} \mathrm{CO}\right), 4.85(\mathrm{~s}, 8 \mathrm{H}$, $\left.\mathrm{OCH}_{2} \mathrm{CO}\right), 7.40(\mathrm{~s}, 8 \mathrm{H}, \mathrm{ArH}), 8.78\left(\mathrm{t},{ }^{3} J_{\mathrm{HH}}=5.1 \mathrm{~Hz}, 4 \mathrm{H}, \mathrm{CONH}\right)$. ${ }^{13} \mathrm{C}$ NMR (100 MHz, $298 \mathrm{~K}, \mathrm{DMSO}-\mathrm{d}_{6}$ ) $\delta: 168.96,164.72,157.65$, 147.04, 134.59, 127.87, 119.26 (q), 74.22, 65.87, 55.90, 55.42, 55.98, 33.91, 32.14, 30.61, 27.40, 27.31, 21.66, 13.77, 7.36. El. anal. calcd for $\mathrm{C}_{108} \mathrm{H}_{164} \mathrm{~F}_{24} \mathrm{~N}_{12} \mathrm{O}_{32} \mathrm{~S}_{12}$ : C $43.48 \%, \mathrm{H} 5.54 \%, \mathrm{~N}$ $5.63 \%$, S $12.90 \%$. Found: C $43.52 \%$, H $5.63 \%$, N $5.55 \%, \mathrm{~S}$ 12.72\%. MS (ESI): calcd for $\left[\mathrm{M}-4 \mathrm{~N}^{-}\left(\mathrm{SO}_{2} \mathrm{CF}_{3}\right)_{2}\right]^{4+} \mathrm{m} / z=465.6$, found $m / z=465.8$. IR $\nu_{\max }: 1672,1746(\mathrm{C}=\mathrm{O}), 2962,3313(\mathrm{NH})$.

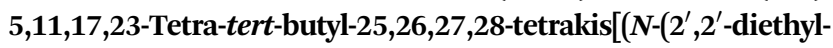
$2^{\prime}-\{($ ethoxycarbonylmethyl)amidocarbonylmethyl $\})$ ammoniumethyl)carbomoylmethoxy]-2,8,14,20-tetrathiacalix[4]arene tetra[bis(trifluoromethylsulfonyl)imide] (cone 53). Yield: $0.13 \mathrm{~g}$ (95\%), mp $66{ }^{\circ} \mathrm{C}^{1}{ }^{1} \mathrm{H}$ NMR (400 MHz, $298 \mathrm{~K}, \mathrm{DMSO}_{6}$ ) $\delta: 1.07$ (s, $\left.36 \mathrm{H},\left(\mathrm{CH}_{3}\right)_{3} \mathrm{C}\right), 1.28\left(\mathrm{t},{ }^{3} J_{\mathrm{HH}}=7.1 \mathrm{~Hz}, 12 \mathrm{H}, \mathrm{CH}_{3} \mathrm{CH}_{2} \mathrm{O}-\right), 1.28(\mathrm{t}$, $\left.{ }^{3} J_{\mathrm{HH}}=7.0 \mathrm{~Hz}, 24 \mathrm{H}, \mathrm{CH}_{3} \mathrm{CH}_{2} \mathrm{~N}^{+}\right), 3.52\left(\mathrm{q},{ }^{3} J_{\mathrm{HH}}=7.0 \mathrm{~Hz}, 8 \mathrm{H}\right.$, $\left.\mathrm{CH}_{3} \mathrm{CH}_{2} \mathrm{~N}^{+}\right), 3.55\left(\mathrm{~m}, 8 \mathrm{H}, \mathrm{NHCH}_{2} \mathrm{CH}_{2} \mathrm{~N}^{+}\right), 3.62(\mathrm{~m}, 8 \mathrm{H}$, $\mathrm{NHCH}_{2} \mathrm{CH}_{2} \mathrm{~N}^{+}$), $3.93\left(\mathrm{~d},{ }^{3} \mathrm{~J}_{\mathrm{HH}}=5.6 \mathrm{~Hz}, 8 \mathrm{H}, \mathrm{NHCH}_{2} \mathrm{CO}\right.$ ), 4.10 (q, $\left.{ }^{3} J_{\mathrm{HH}}=7.1 \mathrm{~Hz}, 8 \mathrm{H}, \mathrm{CH}_{3} \mathrm{CH}_{2} \mathrm{O}^{-}\right), 4.11\left(\mathrm{~s}, 8 \mathrm{H}, \mathrm{N}^{+} \mathrm{CH}_{2} \mathrm{CO}\right), 4.83(\mathrm{~s}$, $\left.8 \mathrm{H}, \mathrm{OCH}_{2} \mathrm{CO}\right), 7.39(\mathrm{~s}, 8 \mathrm{H}, \mathrm{ArH}), 8.76\left(\mathrm{t},{ }^{3} J_{\mathrm{HH}}=5.7 \mathrm{~Hz}, 4 \mathrm{H}\right.$, $\left.\mathrm{NHCH}_{2} \mathrm{CH}_{2} \mathrm{~N}^{+}\right), 9.06\left(\mathrm{t},{ }^{3} J_{\mathrm{HH}}=5.6 \mathrm{~Hz}, 4 \mathrm{H}, \mathrm{NHCH}_{2} \mathrm{CO}\right) .{ }^{13} \mathrm{C} \mathrm{NMR}$ (100 MHz, 298 K, DMSO-d $\left.{ }_{6}\right) \delta: 169.06,168.99,163.58,157.58$, 146.81, 134.56, 127.96, 119.43 (q), 74.14, 60.78, 56.34, 56.08, $55.09,40.67,34.04,32.18,30.55,13.98,7.35$. El. anal. calcd for $\mathrm{C}_{108} \mathrm{H}_{160} \mathrm{~F}_{24} \mathrm{~N}_{16} \mathrm{O}_{36} \mathrm{~S}_{12}$ : C 41.85\%, H 5.20\%, N 7.23\%, S $12.42 \%$. Found: C $42.11 \%$, H 4.92\%, N 7.33\%, S 12.12\%. MS (ESI): calcd for $\left[\mathrm{M}-4 \mathrm{~N}^{-}\left(\mathrm{SO}_{2} \mathrm{CF}_{3}\right)_{2}\right]^{4+} \mathrm{m} / z=480.6$, found $\mathrm{m} / z=480.6$. IR $\nu_{\text {max }} 1180$ (COC), $1676(\mathrm{C}=\mathrm{O}), 2965,3353(\mathrm{~N}-\mathrm{H})$.

5,11,17,23-Tetra-tert-butyl-25,26,27,28-tetrakis[ $N$-(2', $2^{\prime}$-diethyl$2^{\prime}-\{([$ ethoxycarbonylmethyl] amidocarbonylmethyl)amidocarbonylmethyl\}ammoniumethyl)carbomoylmethoxy]-2,8,14,20-tetrathiacalix[4] arene tetra[bis(trifluoromethylsulfonyl)imide] (cone 54). Yield: $0.14 \mathrm{~g}(94 \%), \mathrm{mp} 64{ }^{\circ} \mathrm{C}$. ${ }^{1} \mathrm{H}$ NMR (400 MHz, $298 \mathrm{~K}$, DMSO-d $\left._{6}\right) \delta: 1.07\left(\mathrm{~s}, 36 \mathrm{H},\left(\mathrm{CH}_{3}\right)_{3} \mathrm{C}\right), 1.19\left(\mathrm{t},{ }^{3} \mathrm{~J}_{\mathrm{HH}}=7.1 \mathrm{~Hz}, 12 \mathrm{H}\right.$, $\left.\mathrm{CH}_{3} \mathrm{CH}_{2} \mathrm{O}-\right), 1.28\left(\mathrm{t},{ }^{3} \mathrm{~J}_{\mathrm{HH}}=6.7 \mathrm{~Hz}, 24 \mathrm{H}, \mathrm{CH}_{3} \mathrm{CH}_{2} \mathrm{~N}^{+}\right), 3.50-3.58$ $\left(\mathrm{m}, 24 \mathrm{H}, \mathrm{CH}_{3} \mathrm{CH}_{2} \mathrm{~N}^{+}, \mathrm{NHCH}_{2} \mathrm{CH}_{2} \mathrm{~N}^{+}\right), 3.62\left(\mathrm{~m}, 8 \mathrm{H}, \mathrm{NHCH}_{2}-\right.$ $\left.\mathrm{CH}_{2} \mathrm{~N}^{+}\right), 3.84\left(\mathrm{~d},{ }^{3} \mathrm{~J}_{\mathrm{HH}}=5.9 \mathrm{~Hz}, 8 \mathrm{H}, \mathrm{NHCH}_{2} \mathrm{CO}\right), 3.87\left(\mathrm{~d},{ }^{3} J_{\mathrm{HH}}=\right.$ $\left.6.0 \mathrm{~Hz}, 8 \mathrm{H}, \mathrm{NHCH}_{2} \mathrm{CO}\right), 4.08\left(\mathrm{~s}, 8 \mathrm{H}, \mathrm{N}^{+} \mathrm{CH}_{2} \mathrm{CO}\right), 4.10\left(\mathrm{q},{ }^{3} J_{\mathrm{HH}}=\right.$ $\left.7.1 \mathrm{~Hz}, 8 \mathrm{H}, \mathrm{CH}_{3} \mathrm{CH}_{2} \mathrm{O}\right), 4.83$ (s, 8H, $\left.\mathrm{OCH}_{2} \mathrm{CO}\right), 7.45$ (s, 8H, ArH), $8.50\left(\mathrm{t},{ }^{3} J_{\mathrm{HH}}=5.9 \mathrm{~Hz}, 4 \mathrm{H}, \mathrm{NHCH}_{2} \mathrm{CH}_{2} \mathrm{~N}^{+}\right), 8.74$ (br. s, $4 \mathrm{H}$, $\mathrm{CONH}), 8.87$ (br. s, 4H, CONH). ${ }^{13} \mathrm{C}$ NMR (100 MHz, $298 \mathrm{~K}$, DMSO-d $_{6}$ ) $\delta$ : 170.10, 169.34, 168.80, 163.67, 158.08, 147.57, 135.05, 128.46, 119.57 (q), 74.54, 60.93, 56.99, 56.49, 55.58, $41.87,41.05,34.41,32.68,31.14,14.41,7.89$. El. anal. calcd for $\mathrm{C}_{112} \mathrm{H}_{164} \mathrm{~F}_{24} \mathrm{~N}_{20} \mathrm{O}_{40} \mathrm{~S}_{12}$ : C 41.12\%, H 5.05\%, N 8.56\%, S 11.76\%. Found: C 40.99\%, H 5.07\%, N 8.66\%, S 11.54\%. MS (ESI): calcd for $\left[\mathrm{M}-3 \mathrm{~N}^{-}\left(\mathrm{SO}_{2} \mathrm{CF}_{3}\right)_{2}\right]^{3+} m / z=810.3,\left[\mathrm{M}-4 \mathrm{~N}^{-}\left(\mathrm{SO}_{2} \mathrm{CF}_{3}\right)_{2}\right]^{4+} \mathrm{m} /$ $z=537.6$, found $m / z=810.3$, 537.8. IR $\nu_{\text {max }}$ : 1182 (COC), 1665 $(\mathrm{C}=\mathrm{O}), 2966,3332(\mathrm{~N}-\mathrm{H})$.

5,11,17,23-Tetra-tert-butyl-25,26,27,28-tetrakis[ $N$-(2', $2^{\prime}$-diethyl$2^{\prime}-\{($ ethoxycarbonyl $[S$-methyl $]$ methyl)amidocarbonylmethyl $\}$ ammoniumethyl)carbomoylmethoxy]-2,8,14,20-tetrathiacalix[4] arene tetra[bis(trifluoromethylsulfonyl)imide] (cone 55). Yield: $0.12 \mathrm{~g}(92 \%), \mathrm{mp} 63{ }^{\circ} \mathrm{C} .{ }^{1} \mathrm{H}$ NMR $\left(400 \mathrm{MHz}, 298 \mathrm{~K}, \mathrm{DMSO}_{6}\right) \delta$ : $1.08\left(\mathrm{~s}, 36 \mathrm{H},\left(\mathrm{CH}_{3}\right)_{3} \mathrm{C}\right), 1.18\left(\mathrm{t},{ }^{3} \mathrm{~J}_{\mathrm{HH}}=7.1 \mathrm{~Hz}, 12 \mathrm{H}, \mathrm{CH}_{3} \mathrm{CH}_{2} \mathrm{O}-\right)$, $1.30\left(\mathrm{~d},{ }^{3} J_{\mathrm{HH}}=7.3 \mathrm{~Hz}, 12 \mathrm{H}, \mathrm{CH}_{3} \mathrm{CH}\right), 1.31\left(\mathrm{t},{ }^{3} J_{\mathrm{HH}}=7.2 \mathrm{~Hz}, 24 \mathrm{H}\right.$, $\mathrm{CH}_{3} \mathrm{CH}_{2} \mathrm{~N}^{+}$), 3.48-3.61 (m, 24H, $\mathrm{NHCH}_{2} \mathrm{CH}_{2} \mathrm{~N}^{+}, \mathrm{N}^{+} \mathrm{CH}_{2} \mathrm{CH}_{3}$ ), $3.63\left(\mathrm{~m}, 8 \mathrm{H}, \mathrm{NHCH}_{2} \mathrm{CH}_{2} \mathrm{~N}^{+}\right), 4.09\left(\mathrm{~s}, 8 \mathrm{H}, \mathrm{N}^{+} \mathrm{CH}_{2} \mathrm{CO}\right), 4.11(\mathrm{~m}$, $8 \mathrm{H}, \mathrm{CH}_{3} \mathrm{CH}_{2} \mathrm{O}-$ ), 4.27 (m, $4 \mathrm{H}, \mathrm{CH}_{3} \mathrm{CH}$ ), 4.84 (s, 8H, $\mathrm{OCH}_{2} \mathrm{CO}$ ), $7.40(\mathrm{~s}, 8 \mathrm{H}, \mathrm{ArH}), 8.81$ (br. s, $\left.4 \mathrm{H}, \mathrm{NHCH}_{2} \mathrm{CH}_{2} \mathrm{~N}^{+}\right), 9.23\left(\mathrm{~d},{ }^{3} J_{\mathrm{HH}}=\right.$ $6.7 \mathrm{~Hz}, 4 \mathrm{H}, \mathrm{CONH}) .{ }^{13} \mathrm{C}$ NMR (100 MHz, $\left.298 \mathrm{~K}, \mathrm{DMSO}_{6}\right) \delta$ : $171.74,168.94,162.97,157.56,146.83,134.55,127.82,119.46$ (q), 73.98, 60.75, 56.15, 55.17, 55.07, 47.64, 33.95, 32.19, 30.55, 16.56, 13.96, 7.35. El. anal. calcd for $\mathrm{C}_{108} \mathrm{H}_{160} \mathrm{~F}_{24} \mathrm{~N}_{16} \mathrm{O}_{36} \mathrm{~S}_{12}$ : C $41.85 \%$, H $5.20 \%$, N 7.23\%, S $12.42 \%$. Found: C $42.01 \%, \mathrm{H}$ $5.17 \%, \mathrm{~N} 7.43 \%$, S $12.17 \%$. MS (ESI): calcd for $\left[\mathrm{M}-4 \mathrm{~N}^{-}\left(\mathrm{SO}_{2}{ }^{-}\right.\right.$ $\left.\left.\mathrm{CF}_{3}\right)_{2}\right]^{4+} m / z=494.6$, found $m / z=494.3$. IR $\nu_{\text {max }}: 1183$ (COC), $1680(\mathrm{C}=\mathrm{O}), 2966,3347(\mathrm{~N}-\mathrm{H})$.

$5,11,17,23$-Tetra-tert-butyl-25,26,27, 28-tetrakis $\left[\left(N-\left(3^{\prime}, 3^{\prime}, 3^{\prime}-\right.\right.\right.$ trimethyl)ammoniumpropyl)carbomoylmethoxy]-2,8,14,20-tetrathiacalix[4] arene tetra[bis(trifluoromethylsulfonyl)imide] (1,3alternate 56). Yield: $0.11 \mathrm{~g}(95 \%), \mathrm{mp} 106{ }^{\circ} \mathrm{C} .{ }^{1} \mathrm{H} \mathrm{NMR}(400 \mathrm{MHz}$, $298 \mathrm{~K}$, DMSO-d $\left.{ }_{6}\right) \delta: 1.21\left(\mathrm{~s}, 36 \mathrm{H},\left(\mathrm{CH}_{3}\right)_{3} \mathrm{C}\right), 1.90(\mathrm{~m}, 8 \mathrm{H}$, $\left.-\mathrm{NCH}_{2} \mathrm{CH}_{2} \mathrm{CH}_{2} \mathrm{NH}\right), 3.06\left(\mathrm{~s}, 36 \mathrm{H},\left(\mathrm{CH}_{3}\right)_{3} \mathrm{~N}^{+}\right), 3.18(\mathrm{~m}, 8 \mathrm{H}$, $-\mathrm{NCH}_{2} \mathrm{CH}_{2} \mathrm{CH}_{2} \mathrm{NH}$ ), 3.31 (m, 8H, $\left.\mathrm{NCH}_{2} \mathrm{CH}_{2} \mathrm{CH}_{2} \mathrm{NH}\right), 4.00$ (s, 8H, $\mathrm{OCH}_{2} \mathrm{CO}$ ), 7.60 (s, 8H, ArH), 8.03 (br. s, 4H, CONH). ${ }^{13} \mathrm{C}$ NMR (100 MHz, $298 \mathrm{~K}, \mathrm{DMSO}_{6}$ ) $\delta:$ 167.09, 157.35, 145.86, 133.12, 127.57, 119.75 (q), 70.93, 63.31, 52.37, 35.76, 33.95, 30.58, 22.94. El. anal. calcd for $\mathrm{C}_{80} \mathrm{H}_{116} \mathrm{~F}_{24} \mathrm{~N}_{12} \mathrm{O}_{24} \mathrm{~S}_{12}$ : C $38.89 \%, \mathrm{H} 4.73 \%, \mathrm{~N}$ $6.80 \%, \mathrm{~S} 15.57 \%$. Found: C $39.03 \%, \mathrm{H} 4.91 \%, \mathrm{~N} 6.73 \%, \mathrm{~S}$ 15.72\%. MS (MALDI TOF): calcd for $\left[\mathrm{M}-\mathrm{N}^{-}\left(\mathrm{SO}_{2} \mathrm{CF}_{3}\right)_{2}\right]^{+} \mathrm{m} / \mathrm{z}=$ 2190.4, found $m / z=2190.1$. IR $\nu_{\max }: 1669(\mathrm{C}=\mathrm{O}), 2966$, $3313(\mathrm{NH})$.

$5,11,17,23-T e t r a-t e r t-b u t y l-25,26,27,28$-tetrakis $\left[\left(N-\left(3^{\prime}, 3^{\prime}\right.\right.\right.$-dimethyl-3'-ethyl)ammoniumpropyl)carbomoylmethoxy]-2,8,14,20tetrathiacalix[4]arene tetra[bis(trifluoromethylsulfonyl)imide] (1,3-alternate 57). Yield: $0.12 \mathrm{~g}$ (96\%), mp $96{ }^{\circ} \mathrm{C}$. ${ }^{1} \mathrm{H}$ NMR (400 $\left.\mathrm{MHz}, 298 \mathrm{~K}, \mathrm{DMSO}_{6}\right) \delta: 1.21\left(\mathrm{~s}, 36 \mathrm{H},\left(\mathrm{CH}_{3}\right)_{3} \mathrm{C}\right), 1.24\left(\mathrm{t},{ }^{3} J_{\mathrm{HH}}=\right.$ $\left.7.0 \mathrm{~Hz}, 12 \mathrm{H}, \mathrm{N}^{+} \mathrm{CH}_{2} \mathrm{CH}_{3}\right), 1.87\left(\mathrm{~m}, 8 \mathrm{H},-\mathrm{NCH}_{2} \mathrm{CH}_{2} \mathrm{CH}_{2} \mathrm{NH}\right), 2.99$ $\left(\mathrm{s}, 24 \mathrm{H},\left(\mathrm{CH}_{3}\right)_{2} \mathrm{~N}^{+}\right), 3.19\left(\mathrm{~m}, 8 \mathrm{H},-\mathrm{NCH}_{2} \mathrm{CH}_{2} \mathrm{CH}_{2} \mathrm{NH}\right), 3.24(\mathrm{~m}$, $8 \mathrm{H}, \mathrm{NCH}_{2} \mathrm{CH}_{2} \mathrm{CH}_{2} \mathrm{NH}$ ), 3.33 (q, ${ }^{3} J_{\mathrm{HH}}=7.1 \mathrm{~Hz}, 8 \mathrm{H}, \mathrm{N}^{+} \mathrm{CH}_{2} \mathrm{CH}_{3}$ ), 4.00 (s, 8H, $\mathrm{OCH}_{2} \mathrm{CO}$ ), 7.60 (s, 8H, ArH), 8.03 (br. s, 4H, CONH). ${ }^{13} \mathrm{C}$ NMR (100 MHz, $298 \mathrm{~K}, \mathrm{DMSO}_{6}$ ) $\delta: 167.18,157.05,145.99$, 133.07, 127.45, 119.90 (q), 73.72, 60.28, 58.67, 49.44, 35.92, 33.85 , 30.75, 22.43, 7.76. El. anal. calcd for $\mathrm{C}_{84} \mathrm{H}_{124} \mathrm{~F}_{24} \mathrm{~N}_{12} \mathrm{O}_{24} \mathrm{~S}_{12}$ : C $39.93 \%$, H $4.95 \%$, N 6.65\%, S $15.23 \%$. Found: C $39.74 \%, \mathrm{H}$ $5.10 \%, \mathrm{~N} 6.78 \%, \mathrm{~S} 15.25 \%$. MS (MALDI TOF): calcd for [M $\left.\mathrm{N}^{-}\left(\mathrm{SO}_{2} \mathrm{CF}_{3}\right)_{2}\right]^{+} m / z=2244.6$, found $m / z=2244.8$. IR $\nu_{\text {max }}: 1669$ $(\mathrm{C}=\mathrm{O}), 2966,3310(\mathrm{NH})$.

$5,11,17,23-$ Tetra-tert-butyl-25,26,27,28-tetrakis $\left[\left(N-\left(3^{\prime}, 3^{\prime}\right.\right.\right.$-dimethyl-3'-benzyl)ammoniumpropyl)carbomoylmethoxy]-2,8,14,20tetrathiacalix[4]arene tetra[bis(trifluoromethylsulfonyl)imide] (1,3-alternate 58). Yield: $0.11 \mathrm{~g}$ (94\%), mp $87{ }^{\circ} \mathrm{C}$. ${ }^{1} \mathrm{H}$ NMR (400 MHz, $298 \mathrm{~K}, \mathrm{DMSO}_{6}$ ) $\delta: 1.20$ (s, 36H, $\left.\left(\mathrm{CH}_{3}\right)_{3} \mathrm{C}\right), 2.04(\mathrm{~m}, 8 \mathrm{H}$, $\left.-\mathrm{NCH}_{2} \mathrm{CH}_{2} \mathrm{CH}_{2} \mathrm{NH}\right), 2.97\left(\mathrm{~s}, 24 \mathrm{H},\left(\mathrm{CH}_{3}\right)_{2} \mathrm{~N}^{+}\right), 3.22(\mathrm{~m}, 8 \mathrm{H}$, $-\mathrm{NCH}_{2} \mathrm{CH}_{2} \mathrm{CH}_{2} \mathrm{NH}$ ), 3.27 (m, 8H, $\left.\mathrm{NCH}_{2} \mathrm{CH}_{2} \mathrm{CH}_{2} \mathrm{NH}\right), 4.03$ (s, 8H, $\mathrm{OCH}_{2} \mathrm{CO}$ ), 4.53 (s, 8H, N ${ }^{+} \mathrm{CH}_{2} \mathrm{CO}$ ), 7.51-7.57 (m, 20H, Ar' $\mathrm{H}$ ), 7.60 (s, 8H, ArH), 8.07 (br. s, 4H, CONH). ${ }^{13} \mathrm{C} \mathrm{NMR} \mathrm{(100} \mathrm{MHz,} 298 \mathrm{~K}$, DMSO-d $_{6}$ ) $\delta: 167.62,157.18,145.98,133.09,132.91,130.34$, 128.93, 127.91, 127.49, 119.34 (q), 71.01, 66.16, 61.13, $49.32,35.85,33.85,30.76,22.63$. El. anal. calcd for $\mathrm{C}_{104} \mathrm{H}_{132} \mathrm{~F}_{24} \mathrm{~N}_{12} \mathrm{O}_{24} \mathrm{~S}_{12}$ : C $45.01 \%$, H $4.79 \%$, N 6.06\%, S $13.87 \%$. 
Found: C 44.96\%, H 4.99\%, N 5.90\%, S 13.62\%. MS (MALDI TOF): calcd for $\left[\mathrm{M}-\mathrm{N}^{-}\left(\mathrm{SO}_{2} \mathrm{CF}_{3}\right)_{2}\right]^{+} \mathrm{m} / z=2495.8$, found $\mathrm{m} / z=$ 2495.3. IR $\nu_{\max }: 1672(\mathrm{C}=\mathrm{O}), 2965,3374(\mathrm{NH})$.

$5,11,17,23-T e t r a-t e r t-b u t y l-25,26,27,28$-tetrakis $\left[N-\left(3^{\prime}, 3^{\prime}\right.\right.$-dimethyl3 '-(ethoxycarbonylmethyl)ammoniumpropyl)carbomoylmethoxy]2,8,14,20-tetrathiacalix[4]arene tetra[bis(trifluoromethylsulfonyl)imide] (1,3-alternate 59). Yield: $0.11 \mathrm{~g}(94 \%), \operatorname{mp~} 53{ }^{\circ} \mathrm{C} .{ }^{1} \mathrm{H}$ NMR (400 MHz, $\left.298 \mathrm{~K}, \mathrm{DMSO}_{6}\right) \delta: 1.20\left(\mathrm{~s}, 36 \mathrm{H},\left(\mathrm{CH}_{3}\right)_{3} \mathrm{C}\right), 1.25$ $\left(\mathrm{t},{ }^{3} J_{\mathrm{HH}}=7.1 \mathrm{~Hz}, 12 \mathrm{H}, \mathrm{OCH}_{2} \mathrm{CH}_{3}\right) 1.92\left(\mathrm{~m}, 8 \mathrm{H},-\mathrm{NCH}_{2} \mathrm{CH}_{2}-\right.$ $\left.\mathrm{CH}_{2} \mathrm{NH}\right), 3.18\left(\mathrm{~m}, 8 \mathrm{H},-\mathrm{NCH}_{2} \mathrm{CH}_{2} \mathrm{CH}_{2} \mathrm{NH}\right), 3.20(\mathrm{~s}, 24 \mathrm{H}$, $\left.\left(\mathrm{CH}_{3}\right)_{2} \mathrm{~N}^{+}\right), 3.51\left(\mathrm{~m}, 8 \mathrm{H}, \mathrm{NCH}_{2} \mathrm{CH}_{2} \mathrm{CH}_{2} \mathrm{NH}\right), 4.00(\mathrm{~s}, 8 \mathrm{H}$, $\mathrm{OCH}_{2} \mathrm{CO}$ ), $4.23\left(\mathrm{q},{ }^{3} \mathrm{~J}_{\mathrm{HH}}=7.2 \mathrm{~Hz}, 8 \mathrm{H}, \mathrm{N}^{+} \mathrm{CH}_{2} \mathrm{CH}_{3}\right), 4.42(\mathrm{~s}, 8 \mathrm{H}$, $\mathrm{N}^{+} \mathrm{CH}_{2} \mathrm{CO}$ ), 7.59 (s, 8H, ArH), 8.02 (br. s, 4H, CONH). ${ }^{13} \mathrm{C}$ NMR (100 MHz, 298 K, DMSO-d ${ }_{6}$ ) $\delta: 167.40,164.74,157.11,146.00$, 132.97, 127.54, 119.46 (q), 70.93, 62.32, 61.86, 60.54, 51.21, $35.68,33.83,30.73,22.52$, 13.75. El. anal. calcd for $\mathrm{C}_{92} \mathrm{H}_{132} \mathrm{~F}_{24} \mathrm{~N}_{12} \mathrm{O}_{32} \mathrm{~S}_{12}$ : C $40.05 \%$, H $4.82 \%$, N 6.09\%, S $13.95 \%$. Found: C 39.75\%, H 5.19\%, N 5.85\%, S 13.66\%. MS (MALDI TOF): calcd for $\left[\mathrm{M}-\mathrm{N}^{-}\left(\mathrm{SO}_{2} \mathrm{CF}_{3}\right)_{2}\right]^{+} \mathrm{m} / z=2476.6$, found $m / z=$ 2476.9. IR $\nu_{\max }: 1666,1748(\mathrm{C}=\mathrm{O}), 2969,3316(\mathrm{NH})$.

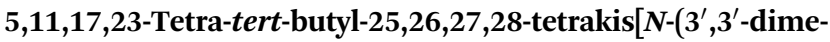
thyl-3'-(pentoxycarbonylmethyl)ammoniumpropyl)carbomoylmethoxy]-2,8,14,20-tetrathiacalix[4]arene tetra[bis(trifluoromethylsulfonyl)imide] (1,3-alternate 60). Yield: $0.13 \mathrm{~g}(92 \%), \mathrm{mp} 47^{\circ} \mathrm{C}$. ${ }^{1} \mathrm{H}$ NMR (400 MHz, $\left.298 \mathrm{~K}, \mathrm{DMSO}_{6}\right) \delta: 0.88\left(\mathrm{t},{ }^{3} J_{\mathrm{HH}}=6.9 \mathrm{~Hz}\right.$, $\left.12 \mathrm{H}, \mathrm{CH}_{2} \mathrm{CH}_{3}\right), 1.20\left(\mathrm{~s}, 36 \mathrm{H},\left(\mathrm{CH}_{3}\right)_{3} \mathrm{C}\right), 1.22-1.30(\mathrm{~m}, 16 \mathrm{H}$, $\left.\mathrm{O}\left(\mathrm{CH}_{2}\right)_{2} \mathrm{CH}_{2} \mathrm{C}_{2} \mathrm{H}_{5}, \mathrm{O}\left(\mathrm{CH}_{2}\right)_{3} \mathrm{CH}_{2} \mathrm{CH}_{3}\right), 1.62\left(\mathrm{~m}, 8 \mathrm{H}, \mathrm{OCH}_{2} \mathrm{CH}_{2} \mathrm{C}_{3}-\right.$ $\left.\mathrm{H}_{7}\right), 1.92\left(\mathrm{~m}, 8 \mathrm{H},-\mathrm{NCH}_{2} \mathrm{CH}_{2} \mathrm{CH}_{2} \mathrm{NH}\right), 3.19\left(\mathrm{~s}, 24 \mathrm{H},\left(\mathrm{CH}_{3}\right)_{2} \mathrm{~N}^{+}\right)$, $3.30\left(\mathrm{~m}, 8 \mathrm{H}, \mathrm{NCH}_{2} \mathrm{CH}_{2} \mathrm{CH}_{2} \mathrm{NH}\right), 3.53\left(\mathrm{~m}, 8 \mathrm{H},-\mathrm{NCH}_{2} \mathrm{CH}_{2} \mathrm{CH}_{2}-\right.$ $\mathrm{NH}), 3.99\left(\mathrm{~s}, 8 \mathrm{H}, \mathrm{OCH}_{2} \mathrm{CO}\right), 4.19\left(\mathrm{t},{ }^{3} J_{\mathrm{HH}}=6.5 \mathrm{~Hz}, 8 \mathrm{H}\right.$, $\mathrm{OCH}_{2} \mathrm{C}_{4} \mathrm{H}_{9}$ ), 4.48 (s, 8H, N ${ }^{+} \mathrm{CH}_{2} \mathrm{CO}$ ), 7.61 (s, 8H, ArH), 8.06 (br. s, $4 \mathrm{H}, \mathrm{CONH}) .{ }^{13} \mathrm{C}$ NMR (100 MHz, $\left.298 \mathrm{~K}, \mathrm{DMSO}_{6}\right) \delta: 167.36$, 164.75, 157.12, 145.98, 133.05, 127.54, 119.58 (q), 70.94, 65.79, $62.31,60.46,51.20,35.67,33.82,30.73,27.42,27.36,22.53$, 21.66, 13.78. El. anal. calcd for $\mathrm{C}_{104} \mathrm{H}_{156} \mathrm{~F}_{24} \mathrm{~N}_{12} \mathrm{O}_{32} \mathrm{~S}_{12}$ : C $42.67 \%$, H 5.37\%, N 5.74\%, S 13.15\%. Found: C 42.55\%, H 5.44\%, N $5.53 \%$, S 12.89\%. MS (ESI): calcd for $\left[\mathrm{M}-3 \mathrm{~N}^{-}\left(\mathrm{SO}_{2} \mathrm{CF}_{3}\right)_{2}\right]^{3+} \mathrm{m} / \mathrm{z}$ $=695.0,\left[\mathrm{M}-4 \mathrm{~N}^{-}\left(\mathrm{SO}_{2} \mathrm{CF}_{3}\right)_{2}\right]^{4+} m / z=451.6$, found $m / z=695.0$, 451.5. IR $\nu_{\text {max }}: 1667,1747(\mathrm{C}=\mathrm{O}), 2962,3312(\mathrm{NH})$.

$5,11,17,23-$ Tetra-tert-butyl-25,26,27,28-tetrakis $\left[N-\left(3^{\prime}, 3^{\prime}\right.\right.$-dimethyl$3^{\prime}$-\{(ethoxycarbonylmethyl)amidocarbonylmethyl\}ammoniumpropyl)carbomoylmethoxy]-2,8,14,20-tetrathiacalix[4]arene tetra[bis(trifluoromethylsulfonyl)imide] (1,3-alternate 61). Yield: $0.13 \mathrm{~g}$ (98\%), mp $73{ }^{\circ} \mathrm{C} .{ }^{1} \mathrm{H}$ NMR (400 MHz, $298 \mathrm{~K}$, DMSO-d $\left.{ }_{6}\right) \delta$ : $1.19\left(\mathrm{t},{ }^{3} \mathrm{~J}_{\mathrm{HH}}=7.1 \mathrm{~Hz}, 12 \mathrm{H}, \mathrm{CH}_{3} \mathrm{CH}_{2} \mathrm{O}-\right), 1.20\left(\mathrm{~s}, 36 \mathrm{H},\left(\mathrm{CH}_{3}\right)_{3} \mathrm{C}\right)$, $1.93\left(\mathrm{~m}, 8 \mathrm{H}, \mathrm{NHCH}_{2} \mathrm{CH}_{2} \mathrm{CH}_{2} \mathrm{~N}^{+}\right), 3.18\left(\mathrm{~s}, 12 \mathrm{H},\left(\mathrm{CH}_{3}\right)_{2} \mathrm{~N}^{+}\right), 3.22$ $\left(\mathrm{m}, 8 \mathrm{H}, \mathrm{NHCH}_{2} \mathrm{CH}_{2} \mathrm{CH}_{2} \mathrm{~N}^{+}\right), 3.50\left(\mathrm{~m}, 8 \mathrm{H}, \mathrm{NHCH}_{2} \mathrm{CH}_{2} \mathrm{CH}_{2} \mathrm{~N}^{+}\right)$, $3.96\left(\mathrm{~d},{ }^{3} \mathrm{~J}_{\mathrm{HH}}=5.7 \mathrm{~Hz}, 8 \mathrm{H}, \mathrm{NHCH}_{2} \mathrm{CO}\right), 3.99\left(\mathrm{~s}, 8 \mathrm{H}, \mathrm{OCH}_{2} \mathrm{CO}\right)$, $4.10\left(\mathrm{q},{ }^{3} \mathrm{~J}_{\mathrm{HH}}=7.1 \mathrm{~Hz}, 8 \mathrm{H}, \mathrm{CH}_{3} \mathrm{CH}_{2} \mathrm{O}-\right), 4.12\left(\mathrm{~s}, 8 \mathrm{H}, \mathrm{N}^{+} \mathrm{CH}_{2} \mathrm{CO}\right)$, $7.59(\mathrm{~s}, 8 \mathrm{H}, \mathrm{ArH}), 8.03\left(\mathrm{t},{ }^{3} \mathrm{~J}_{\mathrm{HH}}=5.4 \mathrm{~Hz}, 4 \mathrm{H}, \mathrm{NHCH}_{2} \mathrm{CH}_{2} \mathrm{CH}_{2} \mathrm{~N}^{+}\right)$, $9.04\left(\mathrm{t},{ }^{3} J_{\mathrm{HH}}=5.7 \mathrm{~Hz}, 4 \mathrm{H}, \mathrm{NHCH}_{2} \mathrm{CO}\right) .{ }^{13} \mathrm{C} \mathrm{NMR}(100 \mathrm{MHz}, 298$ K, DMSO-d $\left.{ }_{6}\right) \delta: 170.79,169.34,167.78,163.90,157.56,147.15$, 131.57, 128.09, 119.34 (q), 71.56, 62.25, 61.13, 60.21, 51.80, 43.30, 40.99, 31.01, 22.80, 14.39. El. anal. calcd for $\mathrm{C}_{100} \mathrm{H}_{144} \mathrm{~F}_{24} \mathrm{~N}_{16} \mathrm{O}_{36} \mathrm{~S}_{12}$ : C 40.21\%, H 4.86\%, N 7.50\%, S $12.88 \%$. Found: C 40.43\%, H 4.97\%, N 7.34\%, S 12.56\%. MS (ESI): calcd for $\left[\mathrm{M}-4 \mathrm{~N}^{-}\left(\mathrm{SO}_{2} \mathrm{CF}_{3}\right)_{2}\right]^{4+} \mathrm{m} / z=466.6$, found $m / z=466.6$. IR $\nu_{\text {max }}$ : 1180 (COC), $1673(\mathrm{C}=\mathrm{O}), 2968,3374(\mathrm{~N}-\mathrm{H})$.

$\mathbf{5 , 1 1 , 1 7 , 2 3 - T e t r a - t e r t - b u t y l - 2 5 , 2 6 , 2 7 , 2 8 - t e t r a k i s [} \mathrm{N}-\left(3^{\prime}, 3^{\prime}\right.$-dimethyl$3^{\prime}-\{([$ ethoxycarbonylmethyl] amidocarbonylmethyl)amidocarbonylmethyl\}ammoniumpropyl)carbomoylmethoxy]-2,8,14,20-tetrathiacalix[4] arene tetra[bis(trifluoromethylsulfonyl)imide] $(1,3$ alternate 62). Yield: $0.14 \mathrm{~g}(97 \%), \mathrm{mp} 76{ }^{\circ} \mathrm{C}$. ${ }^{1} \mathrm{H} \mathrm{NMR}(400 \mathrm{MHz}$, $\left.298 \mathrm{~K}, \mathrm{DMSO}_{6}\right) \delta: 1.18\left(\mathrm{t},{ }^{3} \mathrm{~J}_{\mathrm{HH}}=7.1 \mathrm{~Hz}, 12 \mathrm{H}, \mathrm{CH}_{3} \mathrm{CH}_{2} \mathrm{O}-\right), 1.20$ $\left(\mathrm{s}, 36 \mathrm{H},\left(\mathrm{CH}_{3}\right)_{3} \mathrm{C}\right), 1.93\left(\mathrm{~m}, 8 \mathrm{H}, \mathrm{NHCH}_{2} \mathrm{CH}_{2} \mathrm{CH}_{2} \mathrm{~N}^{+}\right), 3.18(\mathrm{~s}, 12 \mathrm{H}$, $\left.\left(\mathrm{CH}_{3}\right)_{2} \mathrm{~N}^{+}\right), 3.19\left(\mathrm{~m}, 8 \mathrm{H}, \mathrm{NHCH}_{2} \mathrm{CH}_{2} \mathrm{CH}_{2} \mathrm{~N}^{+}\right), 3.49(\mathrm{~m}, 8 \mathrm{H}$, $\mathrm{NHCH}_{2} \mathrm{CH}_{2} \mathrm{CH}_{2} \mathrm{~N}^{+}$), 3.86 (br. s, $8 \mathrm{H}, \mathrm{NHCH}_{2} \mathrm{CO}$ ), 3.87 (br. s, $8 \mathrm{H}$, $\mathrm{NHCH}_{2} \mathrm{CO}$ ), 3.97 (s, 8H, $\left.\mathrm{OCH}_{2} \mathrm{CO}\right), 4.08$ (s, 8H, N $\mathrm{CH}_{2} \mathrm{CO}$ ), 4.09 (q, $\left.{ }^{3} J_{\mathrm{HH}}=7.1 \mathrm{~Hz}, 8 \mathrm{H}, \mathrm{CH}_{3} \mathrm{CH}_{2} \mathrm{O}-\right), 7.59(\mathrm{~s}, 8 \mathrm{H}, \mathrm{ArH}), 8.02\left(\mathrm{t},{ }^{3} \mathrm{~J}_{\mathrm{HH}}\right.$ $\left.=4.9 \mathrm{~Hz}, 4 \mathrm{H}, \mathrm{NHCH}_{2} \mathrm{CH}_{2} \mathrm{CH}_{2} \mathrm{~N}^{+}\right), 8.50\left(\mathrm{t},{ }^{3} \mathrm{~J}_{\mathrm{HH}}=5.7 \mathrm{~Hz}, 4 \mathrm{H}\right.$, $\mathrm{CONH}), 8.86\left(\mathrm{t},{ }^{3} \mathrm{~J}_{\mathrm{HH}}=5.4 \mathrm{~Hz}, 4 \mathrm{H}, \mathrm{CONH}\right) .{ }^{13} \mathrm{C}$ NMR $(100 \mathrm{MHz}$, $\left.298 \mathrm{~K}, \mathrm{DMSO}_{6}\right) \delta: 170.14,168.82,167.93,157.53,146.55$, 133.46, 128.09, 119.32 (q), 71.39, 63.14, 62.53, 60.97, 51.76, $42.03,41.12$, 36.29, 34.34, 31.22, 22.96, 14.52. El. anal. calcd for $\mathrm{C}_{108} \mathrm{H}_{156} \mathrm{~F}_{24} \mathrm{~N}_{20} \mathrm{O}_{40} \mathrm{~S}_{12}$ : C 40.34\%, H 4.89\%, N 8.71\%, S $11.97 \%$. Found: C 40.70\%, H 5.05\%, N 8.92\%, S 11.66\%. MS (ESI): calcd for $\left[\mathrm{M}-3 \mathrm{~N}^{-}\left(\mathrm{SO}_{2} \mathrm{CF}_{3}\right)_{2}\right]^{3+} m / z=791.3,\left[\mathrm{M}-4 \mathrm{~N}^{-}\left(\mathrm{SO}_{2} \mathrm{CF}_{3}\right)_{2}\right]^{4+} \mathrm{m} /$ $z=523.5$, found $m / z=791.3$, 523.6. IR $\nu_{\max }$ : 1182 (COC), 1663 $(\mathrm{C}=\mathrm{O}), 2966,3313(\mathrm{~N}-\mathrm{H})$.

$5,11,17,23-$ Tetra-tert-butyl-25,26,27,28-tetrakis $\left[N-\left(3^{\prime}, 3^{\prime}\right.\right.$-dimethyl$3^{\prime}$-\{(ethoxycarbonyl[S-methyl]methyl)amidocarbonylmethyl $\}$ ammoniumpropyl)carbomoylmethoxy]-2,8,14,20-tetrathiacalix [4] arene tetra[bis(trifluoromethylsulfonyl)imide] (1,3-alternate 63). Yield: $0.13 \mathrm{~g}$ (97\%), mp $60{ }^{\circ} \mathrm{C} .{ }^{1} \mathrm{H}$ NMR (400 MHz, $298 \mathrm{~K}$, DMSO-d $\left._{6}\right) \delta: 1.18\left(\mathrm{t},{ }^{3} J_{\mathrm{HH}}=7.1 \mathrm{~Hz}, 12 \mathrm{H}, \mathrm{CH}_{3} \mathrm{CH}_{2} \mathrm{O}-\right), 1.20(\mathrm{~s}$, $\left.36 \mathrm{H},\left(\mathrm{CH}_{3}\right)_{3} \mathrm{C}\right), 1.33\left(\mathrm{~d},{ }^{3} J_{\mathrm{HH}}=7.3 \mathrm{~Hz}, 12 \mathrm{H}, \mathrm{CH}_{3} \mathrm{CH}\right), 1.93(\mathrm{~m}$, $\left.8 \mathrm{H}, \mathrm{NHCH}_{2} \mathrm{CH}_{2} \mathrm{CH}_{2} \mathrm{~N}^{+}\right), 3.18\left(\mathrm{~s}, 12 \mathrm{H},\left(\mathrm{CH}_{3}\right)_{2} \mathrm{~N}^{+}\right), 3.19(\mathrm{~m}, 8 \mathrm{H}$, $\mathrm{NHCH}_{2} \mathrm{CH}_{2} \mathrm{CH}_{2} \mathrm{~N}^{+}$), $3.51\left(\mathrm{~m}, 8 \mathrm{H}, \mathrm{NHCH}_{2} \mathrm{CH}_{2} \mathrm{CH}_{2} \mathrm{~N}^{+}\right), 4.01$ (s, $8 \mathrm{H}, \mathrm{OCH}_{2} \mathrm{CO}$ ), 4.09 (s, $\left.8 \mathrm{H}, \mathrm{N}^{+} \mathrm{CH}_{2} \mathrm{CO}\right), 4.10$ (q, ${ }^{3} J_{\mathrm{HH}}=7.1 \mathrm{~Hz}$, $\left.8 \mathrm{H}, \mathrm{CH}_{3} \mathrm{CH}_{2} \mathrm{O}-\right), 4.30\left(\mathrm{~m}, 4 \mathrm{H}, \mathrm{CH}_{3} \mathrm{CH}\right), 7.59$ (s, 8H, $\left.\mathrm{ArH}\right), 8.03(\mathrm{t}$, $\left.{ }^{3} J_{\mathrm{HH}}=4.9 \mathrm{~Hz}, 4 \mathrm{H}, \mathrm{NHCH}_{2} \mathrm{CH}_{2} \mathrm{CH}_{2} \mathrm{~N}^{+}\right), 9.04\left(\mathrm{~d},{ }^{3} J_{\mathrm{HH}}=6.5 \mathrm{~Hz}\right.$, $4 \mathrm{H}, \mathrm{CONH}) .{ }^{13} \mathrm{C}$ NMR (100 MHz, $298 \mathrm{~K}, \mathrm{DMSO}_{6}$ ) $\delta: 172.05$, 166.94, 162.72, 157.53, 145.93, 133.83, 127.27, 119.53 (q), 70.65, 62.43, 61.15, 60.62, 51.16, 47.99, 35.62, 33.55, 30.37, 23.09, 16.65, 13.81. El. anal. calcd for $\mathrm{C}_{104} \mathrm{H}_{152} \mathrm{~F}_{24} \mathrm{~N}_{16} \mathrm{O}_{36} \mathrm{~S}_{12}$ : C $41.05 \%$, H 5.03\%, N 7.36\%, S 12.64\%. Found: C 39.86\%, H $4.66 \%, \mathrm{~N}$ $6.96 \%$, S $13.50 \%$. MS (ESI): calcd for $\left[\mathrm{M}-4 \mathrm{~N}^{-}\left(\mathrm{SO}_{2} \mathrm{CF}_{3}\right)_{2}\right]^{4+} \mathrm{m} / \mathrm{z}$ $=480.6$, found $m / z=480.4$. IR $\nu_{\max }: 1181$ (COC), $1679(\mathrm{C}=\mathrm{O})$, 2965, $3357(\mathrm{~N}-\mathrm{H})$.

5,11,17,23-Tetra-tert-butyl-25,26,27,28-tetrakis $\left[N\right.$-( $3^{\prime}, 3^{\prime}$-dimethyl$3^{\prime}-\left\{3^{\prime \prime}\right.$-propylphthalimide $\}$ ammoniumpropyl)carbomoylmethoxy $]$ 2,8,14,20-tetrathiacalix[4]arene tetra[bis(trifluoromethylsulfonyl)imide] (1,3-alternate 64). Yield: $0.13 \mathrm{~g}$ (91\%), mp $87{ }^{\circ} \mathrm{C} .{ }^{1} \mathrm{H}$ NMR (400 MHz, $\left.298 \mathrm{~K}, \mathrm{DMSO}_{6}\right) \delta: 1.21\left(\mathrm{~s}, 36 \mathrm{H},\left(\mathrm{CH}_{3}\right)_{3} \mathrm{C}\right), 1.88$ (m, 8H, $\mathrm{CH}_{2} \mathrm{CH}_{2} \mathrm{CH}_{2} \mathrm{Pht}$ ), 2.05 (m, 8H, $\mathrm{NHCH}_{2} \mathrm{CH}_{2} \mathrm{CH}_{2} \mathrm{~N}^{+}$), 2.99 $\left(\mathrm{s}, 24 \mathrm{H},\left(\mathrm{CH}_{3}\right)_{2} \mathrm{~N}^{+}\right), 3.17\left(\mathrm{~m}, 8 \mathrm{H}, \mathrm{NHCH}_{2} \mathrm{CH}_{2} \mathrm{CH}_{2} \mathrm{~N}^{+}\right), 3.29(\mathrm{~m}$, $8 \mathrm{H}, \mathrm{CH}_{2} \mathrm{CH}_{2} \mathrm{CH}_{2} \mathrm{Pht}$ ), 3.39 (m, 8H, $\mathrm{NHCH}_{2} \mathrm{CH}_{2} \mathrm{CH}_{2} \mathrm{~N}^{+}$), 3.67 (t, ${ }^{3} J_{\mathrm{HH}}=6.2 \mathrm{~Hz}, 8 \mathrm{H}, \mathrm{CH}_{2} \mathrm{CH}_{2} \mathrm{CH}_{2} \mathrm{Pht}$ ), 3.99 (s, 8H, $\mathrm{OCH}_{2} \mathrm{CO}$ ), 7.60 (s, 8H, ArH), 7.84-7.89 (m, 16H, Pht), $8.04\left(\mathrm{t},{ }^{3} J_{\mathrm{HH}}=5.3 \mathrm{~Hz}, 4 \mathrm{H}\right.$, $\mathrm{NHCH}_{2} \mathrm{CH}_{2} \mathrm{CH}_{2} \mathrm{~N}^{+}$). ${ }^{13} \mathrm{C}$ NMR (100 MHz, $298 \mathrm{~K}$, DMSO-d 6 ) $\delta$ : 167.98, 167.32, 157.15, 146.11, 134.41, 133.07, 131.70, 127.54, 123.06, 119.32 (q), 71.01, 61.35, 61.12, 49.86, 35.79, 34.56, 33.84, $30.74,22.48,21.65$. El. anal. calcd for $\mathrm{C}_{120} \mathrm{H}_{144} \mathrm{~F}_{24} \mathrm{~N}_{16} \mathrm{O}_{32} \mathrm{~S}_{12}$ : C 
45.56\%, H 4.59\%, N 7.08\%, S 12.16\%. Found: C 45.45\%, H $4.26 \%$, N 7.01\%, S $12.45 \%$. MS (ESI): calcd for $\left[\mathrm{M}-4 \mathrm{~N}^{-}\left(\mathrm{SO}_{2}-\right.\right.$ $\left.\left.\mathrm{CF}_{3}\right)_{2}\right]^{4+} m / z=480.6$, found $m / z=480.5$. IR $\nu_{\text {max }}: 1668,1708$, $1771(\mathrm{C}=\mathrm{O}), 2967,3314(\mathrm{~N}-\mathrm{H})$.

$5,11,17,23-$ Tetra-tert-butyl-25,26,27,28-tetrakis[ $(\mathrm{N}$-(2'-methyl$2^{\prime}, 2^{\prime}$-diethyl)ammoniumethyl)carbomoylmethoxy]-2,8,14,20tetrathiacalix[4]arene tetra[bis(trifluoromethylsulfonyl)imide] (1,3-alternate 65). Yield: $0.12 \mathrm{~g}(90 \%), \mathrm{mp} 83{ }^{\circ} \mathrm{C} .{ }^{1} \mathrm{H}$ NMR $(400$ $\left.\mathrm{MHz}, 298 \mathrm{~K}, \mathrm{DMSO}-\mathrm{d}_{6}\right) \delta: 1.22\left(\mathrm{~s}, 36 \mathrm{H},\left(\mathrm{CH}_{3}\right)_{3} \mathrm{C}\right), 1.26\left(\mathrm{t},{ }^{3} J_{\mathrm{HH}}=\right.$ $\left.7.1 \mathrm{~Hz}, 24 \mathrm{H},\left(\mathrm{CH}_{3} \mathrm{CH}_{2}-\right)\right), 3.02\left(\mathrm{~s}, 12 \mathrm{H},\left(\mathrm{CH}_{3}\right) \mathrm{N}^{+}\right), 3.21(\mathrm{~m}, 8 \mathrm{H}$, $\left.-\mathrm{NCH}_{2} \mathrm{CH}_{2} \mathrm{NH}\right), 3.37$ (q, $\left.{ }^{3} J_{\mathrm{HH}}=7.1 \mathrm{~Hz}, 16 \mathrm{H},-\mathrm{CH}_{2} \mathrm{CH}_{3}\right), 3.48(\mathrm{~m}$, $8 \mathrm{H}, \mathrm{NCH}_{2} \mathrm{CH}_{2} \mathrm{NH}$ ), 4.05 (s, 8H, $\mathrm{OCH}_{2} \mathrm{CO}$ ), 7.60 (s, 8H, ArH), 8.17 $\left(\mathrm{t},{ }^{3} \mathrm{~J}_{\mathrm{HH}}=5.6 \mathrm{~Hz}, 4 \mathrm{H}, \mathrm{CONH}\right) .{ }^{13} \mathrm{C}$ NMR $(100 \mathrm{MHz}, 298 \mathrm{~K}$, DMSO$\left.\mathrm{d}_{6}\right) \delta: 168.02,156.89,146.04,132.99,127.49,117.85$ (q), 70.54, $56.87,56.16,46.96,33.88,32.13,30.84,7.39$. El. anal. calcd for $\mathrm{C}_{84} \mathrm{H}_{124} \mathrm{~F}_{24} \mathrm{~N}_{12} \mathrm{O}_{24} \mathrm{~S}_{12}$ : C 39.93\%, H 4.95\%, N 6.65\%, S $15.23 \%$. Found: C $40.02 \%$, H $4.99 \%$, N 6.48\%, S 15.03\%. MS (ESI): calcd for $\left[\mathrm{M}-4 \mathrm{~N}^{-}\left(\mathrm{SO}_{2} \mathrm{CF}_{3}\right)_{2}\right]^{4+} \mathrm{m} / z=351.5$, found $m / z=351.3$. IR $\nu_{\text {max }}: 1671(\mathrm{C}=\mathrm{O}), 2969,3375(\mathrm{NH})$.

$5,11,17,23$-Tetra-tert-butyl-25,26,27,28-tetrakis $\left[\left(N-\left(2^{\prime}, 2^{\prime}, 2^{\prime}-\right.\right.\right.$ triethyl)ammoniumethyl)carbomoylmethoxy]-2,8,14,20-tetrathiacalix[4] arene tetra[bis(trifluoromethylsulfonyl)imide] (1,3alternate 66). Yield: $0.11 \mathrm{~g}(92 \%), \mathrm{mp} 79{ }^{\circ} \mathrm{C} .{ }^{1} \mathrm{H} \mathrm{NMR}(400 \mathrm{MHz}$, $\left.298 \mathrm{~K}, \mathrm{DMSO}_{\mathrm{d}}\right) \delta: 1.21\left(\mathrm{~s}, 36 \mathrm{H},\left(\mathrm{CH}_{3}\right)_{3} \mathrm{C}\right), 1.23\left(\mathrm{t},{ }^{3} \mathrm{~J}_{\mathrm{HH}}=7.0 \mathrm{~Hz}\right.$, $\left.36 \mathrm{H}, \mathrm{CH}_{3} \mathrm{CH}_{2}-\right), 3.14\left(\mathrm{~m}, 8 \mathrm{H},-\mathrm{NCH}_{2} \mathrm{CH}_{2} \mathrm{NH}\right), 3.31(\mathrm{~m}, 24 \mathrm{H}$, $-\mathrm{CH}_{2} \mathrm{CH}_{3}$ ), $3.45\left(\mathrm{~m}, 8 \mathrm{H}, \mathrm{NCH}_{2} \mathrm{CH}_{2} \mathrm{NH}\right), 4.04$ (s, 8H, $\mathrm{OCH}_{2} \mathrm{CO}$ ), $7.60(\mathrm{~s}, 8 \mathrm{H}, \mathrm{ArH}), 8.14\left(\mathrm{t},{ }^{3} \mathrm{JHH}_{\mathrm{HH}}=5.7 \mathrm{~Hz}, 4 \mathrm{H}, \mathrm{CONH}\right) .{ }^{13} \mathrm{C} \mathrm{NMR}$ (100 MHz, $298 \mathrm{~K}$, DMSO-d $\left.{ }_{6}\right) \delta: 168.05,154.01,145.92,133.26$, 127.55, 119.67 (q), 70.51, 53.57, 52.46, 33.88, 31.83, 30.84, 7.04. El. anal. calcd for $\mathrm{C}_{88} \mathrm{H}_{132} \mathrm{~F}_{24} \mathrm{~N}_{12} \mathrm{O}_{24} \mathrm{~S}_{12}$ : C 40.92\%, H 5.15\%, N $6.51 \%$, S 14.90\%. Found: C 41.23\%, H 5.13\%, N 6.71\%, S $15.07 \%$. MS (ESI): calcd for $\left[\mathrm{M}-4 \mathrm{~N}^{-}\left(\mathrm{SO}_{2} \mathrm{CF}_{3}\right)_{2}\right]^{4+} \mathrm{m} / z=365.5$, found $m / z=365.4$. IR $\nu_{\max }: 1670(\mathrm{C}=\mathrm{O}), 2964,3374(\mathrm{NH})$.

$5,11,17,23-T e t r a-t e r t-b u t y l-25,26,27,28-t e t r a k i s\left[\left(N-\left(2^{\prime}, 2^{\prime}\right.\right.\right.$-diethyl$2^{\prime}$-benzyl)ammoniumethyl)carbomoylmethoxy]-2,8,14,20-tetrathiacalix[4] arene tetra[bis(trifluoromethylsulfonyl)imide] (1,3alternate 67). Yield: $0.12 \mathrm{~g}(93 \%), \mathrm{mp} 76{ }^{\circ} \mathrm{C} .{ }^{1} \mathrm{H} \mathrm{NMR}(400 \mathrm{MHz}$, $\left.298 \mathrm{~K}, \mathrm{DMSO}_{6}\right) \delta: 1.19\left(\mathrm{~s}, 36 \mathrm{H},\left(\mathrm{CH}_{3}\right)_{3} \mathrm{C}\right), 1.39\left(\mathrm{t},{ }^{3} \mathrm{~J}_{\mathrm{HH}}=7.0 \mathrm{~Hz}\right.$, $24 \mathrm{H},\left(\mathrm{CH}_{3} \mathrm{CH}_{2}-\right)$ ), $3.15\left(\mathrm{~m}, 8 \mathrm{H},-\mathrm{NCH}_{2} \mathrm{CH}_{2} \mathrm{NH}\right), 3.25\left(\mathrm{q},{ }^{3} J_{\mathrm{HH}}=\right.$ $\left.7.0 \mathrm{~Hz}, 16 \mathrm{H},-\mathrm{CH}_{2} \mathrm{CH}_{3}\right), 3.61\left(\mathrm{~m}, 8 \mathrm{H}, \mathrm{NCH}_{2} \mathrm{CH}_{2} \mathrm{NH}\right), 4.20(\mathrm{~s}, 8 \mathrm{H}$, $\mathrm{OCH}_{2} \mathrm{CO}$ ), 4.58 (s, 8H, N ${ }^{+} \mathrm{CH}_{2} \mathrm{Ph}$ ), 7.50-7.56 (m, 20H, Ar' H), 7.61 $(\mathrm{s}, 8 \mathrm{H}, \mathrm{ArH}), 8.34\left(\mathrm{t},{ }^{3} \mathrm{~J}_{\mathrm{HH}}=5.8 \mathrm{~Hz}, 4 \mathrm{H}, \mathrm{CONH}\right) .{ }^{13} \mathrm{C} \mathrm{NMR}(100$ MHz, 298 K, DMSO-d 6 ) $\delta: 168.05,153.77,145.78,133.41,132.68$, 130.43, 129.12, 119.49 (q), 70.82, 60.56, 54.12, 53.17, 33.84, 32.04, 30.85, 11.11, 7.51. El. anal. calcd for $\mathrm{C}_{108} \mathrm{H}_{140} \mathrm{~F}_{24} \mathrm{~N}_{12} \mathrm{O}_{24} \mathrm{~S}_{12}$ : C 45.82\%, H 4.98\%, N 5.94\%, S 13.59\%. Found: C 46.07\%, H 5.28\%, N 5.99\%, S 13.35\%. MS (ESI): calcd for $\left[\mathrm{M}-4 \mathrm{~N}^{-}\left(\mathrm{SO}_{2} \mathrm{CF}_{3}\right)_{2}\right]^{4+} \mathrm{m} / z=427.2$, found $m / z=427.4$. IR $\nu_{\text {max }}: 1671(\mathrm{C}=\mathrm{O}), 2966,3393(\mathrm{NH})$.

$5,11,17,23$-Tetra-tert-butyl-25,26,27,28-tetrakis $\left[N\right.$-(2', $2^{\prime}$-diethyl$2^{\prime}$-(ethoxycarbonylmethyl)ammoniumethyl)carbomoylmethoxy]2,8,14,20-tetrathiacalix[4]arene tetra[bis(trifluoromethylsulfonyl)imide] (1,3-alternate 68). Yield: $0.12 \mathrm{~g}(93 \%), \operatorname{mp~} 58{ }^{\circ} \mathrm{C} .{ }^{1} \mathrm{H}$ NMR (400 MHz, $\left.298 \mathrm{~K}, \mathrm{DMSO}_{6}\right) \delta: 1.21\left(\mathrm{~s}, 36 \mathrm{H},\left(\mathrm{CH}_{3}\right)_{3} \mathrm{C}\right), 1.26$ $\left(\mathrm{t},{ }^{3} J_{\mathrm{HH}}=7.1 \mathrm{~Hz}, 12 \mathrm{H}, \mathrm{OCH}_{2} \mathrm{CH}_{3}\right), 1.28\left(\mathrm{t},{ }^{3} J_{\mathrm{HH}}=7.0 \mathrm{~Hz}, 24 \mathrm{H}\right.$, $\left.\mathrm{CH}_{3} \mathrm{CH}_{2}-\right)$, 3.45-3.60 (m, 32H, $-\mathrm{CH}_{2} \mathrm{CH}_{3},-\mathrm{NCH}_{2} \mathrm{CH}_{2} \mathrm{NH}$, $\left.-\mathrm{NCH}_{2} \mathrm{CH}_{2} \mathrm{NH}\right), 4.08$ (s, 8H, $\left.\mathrm{OCH}_{2} \mathrm{CO}\right), 4.24$ (q, ${ }^{3} \mathrm{~J}_{\mathrm{HH}}=7.1 \mathrm{~Hz}$, $\left.8 \mathrm{H}, \mathrm{OCH}_{2} \mathrm{CH}_{3}\right), 4.46\left(\mathrm{~s}, 8 \mathrm{H}, \mathrm{N}^{+} \mathrm{CH}_{2} \mathrm{CO}\right), 7.61(\mathrm{~s}, 8 \mathrm{H}, \mathrm{ArH}), 8.22(\mathrm{t}$, $\left.{ }^{3} J_{\mathrm{HH}}=4.7 \mathrm{~Hz}, 4 \mathrm{H}, \mathrm{CONH}\right) .{ }^{13} \mathrm{C}$ NMR $\left(100 \mathrm{MHz}, 298 \mathrm{~K}, \mathrm{DMSO}_{6}\right)$ $\delta: 168.04,164.63,157.00,145.99,133.07,127.40,119.46$ (q), 70.60, 62.13, 55.83, 55.53, 55.08, 33.85, 31.98, 30.85, 13.73, 7.36. El. anal. calcd for $\mathrm{C}_{96} \mathrm{H}_{140} \mathrm{~F}_{24} \mathrm{~N}_{12} \mathrm{O}_{32} \mathrm{~S}_{12}$ : C 40.96\%, H $5.01 \%, \mathrm{~N}$ $5.97 \%$, S 13.67\%. Found: C 41.12\%, H 4.95\%, N 5.99\%, S 13.55\%. MS (ESI): calcd for $\left[\mathrm{M}-4 \mathrm{~N}^{-}\left(\mathrm{SO}_{2} \mathrm{CF}_{3}\right)_{2}\right]^{4+} \mathrm{m} / z=423.6$, found $m / z=423.5$. IR $\nu_{\max }: 1670,1747(\mathrm{C}=\mathrm{O}), 2967,3374(\mathrm{NH})$.

$5,11,17,23$-Tetra-tert-butyl-25,26,27,28-tetrakis $\left[N\right.$-( $2^{\prime}, 2^{\prime}$-diethyl2 -(pentoxycarbonylmethyl)ammoniumethyl)carbomoylmethoxy]2,8,14,20-tetrathiacalix[4]arene tetra[bis(trifluoromethylsulfonyl)imide] (1,3-alternate 69). Yield: $0.11 \mathrm{~g}$ (91\%), mp $49{ }^{\circ} \mathrm{C} .{ }^{1} \mathrm{H}$ NMR (400 MHz, $\left.298 \mathrm{~K}, \mathrm{DMSO}_{6}\right) \delta: 0.87\left(\mathrm{t},{ }^{3} \mathrm{~J}_{\mathrm{HH}}=6.6 \mathrm{~Hz}, 12 \mathrm{H}\right.$, $\left.-\mathrm{O}\left(\mathrm{CH}_{2}\right)_{4} \mathrm{CH}_{3}\right), 1.21\left(\mathrm{~s}, 36 \mathrm{H},\left(\mathrm{CH}_{3}\right)_{3} \mathrm{C}\right), 1.27-1.35(\mathrm{~m}, 16 \mathrm{H}$, $\left.\mathrm{O}\left(\mathrm{CH}_{2}\right)_{3} \mathrm{CH}_{2} \mathrm{CH}_{3}, \mathrm{OCH}_{2} \mathrm{CH}_{2} \mathrm{C}_{3} \mathrm{H}_{7}\right), 1.28\left(\mathrm{t},{ }^{3} \mathrm{~J}_{\mathrm{HH}}=7.0 \mathrm{~Hz}, 24 \mathrm{H}\right.$, $\left.\left(\mathrm{CH}_{3} \mathrm{CH}_{2} \mathrm{~N}^{+}-\right)\right), 1.65\left(\mathrm{~m}, 8 \mathrm{H}, \mathrm{O}\left(\mathrm{CH}_{2}\right)_{2} \mathrm{CH}_{2} \mathrm{C}_{2} \mathrm{H}_{5}\right), 3.44-3.65(\mathrm{~m}$, $\left.32 \mathrm{H},-\mathrm{N}^{+} \mathrm{CH}_{2} \mathrm{CH}_{3},-\mathrm{NCH}_{2} \mathrm{CH}_{2} \mathrm{NH},-\mathrm{NCH}_{2} \mathrm{CH}_{2} \mathrm{NH}\right), 4.07$ (s, 8H, $\mathrm{OCH}_{2} \mathrm{CO}$ ), 4.19 (q, $\left.{ }^{3} J_{\mathrm{HH}}=6.5 \mathrm{~Hz}, 8 \mathrm{H}, \mathrm{OCH}_{2} \mathrm{C}_{4} \mathrm{H}_{9}\right), 4.48(\mathrm{~s}, 8 \mathrm{H}$, $\mathrm{N}^{+} \mathrm{CH}_{2} \mathrm{CO}$ ), 7.69 (s, 8H, ArH), $8.22\left(\mathrm{t},{ }^{3} \mathrm{~J}_{\mathrm{HH}}=5.1 \mathrm{~Hz}, 4 \mathrm{H}, \mathrm{CONH}\right)$. ${ }^{13} \mathrm{C}$ NMR (100 MHz, $298 \mathrm{~K}$, DMSO-d ${ }_{6}$ ) $\delta: 168.01,164.77,156.91$, 146.00, 133.00, 127.45, 119.47 (q), 70.55, 65.90, 55.78, 55.42, 54.99, 33.84, 31.96, 30.83, 27.41, 27.35, 21.66, 13.79, 7.34. El. anal. calcd for $\mathrm{C}_{108} \mathrm{H}_{164} \mathrm{~F}_{24} \mathrm{~N}_{12} \mathrm{O}_{32} \mathrm{~S}_{12}$ : C $43.48 \%, \mathrm{H} 5.54 \%, \mathrm{~N}$ $5.63 \%$, S $12.90 \%$. Found: C $43.71 \%$, H $5.52 \%, \mathrm{~N} 5.77 \%, \mathrm{~S}$ 12.68\%. MS (ESI): calcd for $\left[\mathrm{M}-4 \mathrm{~N}^{-}\left(\mathrm{SO}_{2} \mathrm{CF}_{3}\right)_{2}\right]^{4+} \mathrm{m} / z=465.6$, found $m / z=465.5$. IR $\nu_{\max }: 1671,1746(\mathrm{C}=\mathrm{O}), 2961,3373(\mathrm{NH})$.

$5,11,17,23-$ Tetra-tert-butyl-25,26,27,28-tetrakis $\left[\left(N\right.\right.$ - $\left(2^{\prime}, 2^{\prime}\right.$-diethyl$2^{\prime}-\{($ ethoxycarbonylmethyl)amidocarbonylmethyl $\})$ ammoniumethyl)carbomoylmethoxy]-2,8,14,20-tetrathiacalix[4]arene tetra [bis(trifluoromethylsulfonyl)imide] (1,3-alternate 70). Yield: $0.13 \mathrm{~g}(93 \%), \operatorname{mp} 62{ }^{\circ} \mathrm{C} .{ }^{1} \mathrm{H}$ NMR (400 MHz, $298 \mathrm{~K}$, DMSO-d 6 ) $\delta$ : $1.20\left(\mathrm{t},{ }^{3} J_{\mathrm{HH}}=7.1 \mathrm{~Hz}, 12 \mathrm{H}, \mathrm{CH}_{3} \mathrm{CH}_{2} \mathrm{O}-\right), 1.21\left(\mathrm{~s}, 36 \mathrm{H},\left(\mathrm{CH}_{3}\right)_{3} \mathrm{C}\right)$, $1.31\left(\mathrm{t},{ }^{3} J_{\mathrm{HH}}=7.0 \mathrm{~Hz}, 24 \mathrm{H}, \mathrm{CH}_{3} \mathrm{CH}_{2} \mathrm{~N}^{+}\right), 3.48\left(\mathrm{~m}, 8 \mathrm{H}, \mathrm{NHCH}_{2^{-}}\right.$ $\mathrm{CH}_{2} \mathrm{~N}^{+}$), 3.53-3.59 (m, $\left.16 \mathrm{H}, \mathrm{CH}_{3} \mathrm{CH}_{2} \mathrm{~N}^{+}, \mathrm{NHCH}_{2} \mathrm{CH}_{2} \mathrm{~N}^{+}\right), 3.96$ (d, $\left.{ }^{3} J_{\mathrm{HH}}=5.7 \mathrm{~Hz}, 8 \mathrm{H}, \mathrm{NHCH}_{2} \mathrm{CO}\right), 4.05\left(\mathrm{~s}, 8 \mathrm{H}, \mathrm{OCH}_{2} \mathrm{CO}\right), 4.12$ (q, $\left.{ }^{3} J_{\mathrm{HH}}=7.0 \mathrm{~Hz}, 8 \mathrm{H}, \mathrm{CH}_{3} \mathrm{CH}_{2} \mathrm{O}-\right), 4.11\left(\mathrm{~s}, 8 \mathrm{H}, \mathrm{N}^{+} \mathrm{CH}_{2} \mathrm{CO}\right), 7.60(\mathrm{~s}$, $8 \mathrm{H}, \mathrm{ArH}), 8.22\left(\mathrm{t},{ }^{3} J_{\mathrm{HH}}=5.4 \mathrm{~Hz}, 4 \mathrm{H}, \mathrm{NHCH}_{2} \mathrm{CH}_{2} \mathrm{~N}^{+}\right), 9.10\left(\mathrm{t},{ }^{3} J_{\mathrm{HH}}\right.$ $\left.=5.7 \mathrm{~Hz}, 4 \mathrm{H}, \mathrm{NHCH}_{2} \mathrm{CO}\right) .{ }^{13} \mathrm{C} \mathrm{NMR}\left(100 \mathrm{MHz}, 298 \mathrm{~K}, \mathrm{DMSO}_{6}\right)$ $\delta: 169.05,168.06,163.55,156.85,145.92,132.80,127.65,119.46$ (q), 70.12, 60.85, 56.37, 55.87, 55.10, 40.71, 33.84, 31.95, 30.82, 14.00, 7.36. El. anal. calcd for $\mathrm{C}_{108} \mathrm{H}_{160} \mathrm{~F}_{24} \mathrm{~N}_{16} \mathrm{O}_{36} \mathrm{~S}_{12}$ : C $41.85 \%$, H $5.20 \%$, N 7.23\%, S 12.42\%. Found: C 41.99\%, H 5.31\%, N 7.24\%, S 12.05\%. MS (ESI): calcd for $\left[\mathrm{M}-4 \mathrm{~N}^{-}\left(\mathrm{SO}_{2} \mathrm{CF}_{3}\right)_{2}\right]^{4+} \mathrm{m} / \mathrm{z}$ $=480.6$, found $m / z=481.0$. IR $\nu_{\max }: 1180$ (COC), $1685(\mathrm{C}=\mathrm{O})$, 2967, $3364(\mathrm{~N}-\mathrm{H})$.

$5,11,17,23-T e t r a-t e r t-b u t y l-25,26,27,28$-tetrakis $\left[N-\left(2^{\prime}, 2^{\prime}\right.\right.$-diethyl$2^{\prime}-\{([$ ethoxycarbonylmethyl] amidocarbonylmethyl)amidocarbonylmethyl\}ammoniumethyl)carbomoylmethoxy]-2,8,14,20-tetrathiacalix[4] arene tetra[bis(trifluoromethylsulfonyl)imide] (1,3alternate 71). Yield: $0.14 \mathrm{~g}$ (94\%), mp $64{ }^{\circ} \mathrm{C}$. ${ }^{1} \mathrm{H} \mathrm{NMR}(400 \mathrm{MHz}$, $\left.298 \mathrm{~K}, \mathrm{DMSO}_{6}\right) \delta: 1.19\left(\mathrm{t},{ }^{3} \mathrm{~J}_{\mathrm{HH}}=7.1 \mathrm{~Hz}, 12 \mathrm{H}, \mathrm{CH}_{3} \mathrm{CH}_{2} \mathrm{O}-\right), 1.21$ $\left(\mathrm{s}, 36 \mathrm{H},\left(\mathrm{CH}_{3}\right)_{3} \mathrm{C}\right), 1.30\left(\mathrm{t},{ }^{3} J_{\mathrm{HH}}=7.1 \mathrm{~Hz}, 24 \mathrm{H}, \mathrm{CH}_{3} \mathrm{CH}_{2} \mathrm{~N}^{+}\right), 3.44$ $\left(\mathrm{m}, 8 \mathrm{H}, \mathrm{NHCH}_{2} \mathrm{CH}_{2} \mathrm{~N}^{+}\right), 3.51-3.58\left(\mathrm{~m}, 24 \mathrm{H}, \mathrm{CH}_{3} \mathrm{CH}_{2} \mathrm{~N}^{+}\right.$, $\mathrm{NHCH}_{2} \mathrm{CH}_{2} \mathrm{~N}^{+}$), 3.87 (br. s, $8 \mathrm{H}, \mathrm{NHCH}_{2} \mathrm{CO}$ ), 3.88 (br. s, $8 \mathrm{H}$, $\mathrm{NHCH}_{2} \mathrm{CO}$ ), 4.04 (s, 8H, $\left.\mathrm{OCH}_{2} \mathrm{CO}\right), 4.08\left(\mathrm{q},{ }^{3} J_{\mathrm{HH}}=7.1 \mathrm{~Hz}, 8 \mathrm{H}\right.$, $\mathrm{CH}_{3} \mathrm{CH}_{2} \mathrm{O}^{-}$), 4.09 (s, 8H, N ${ }^{+} \mathrm{CH}_{2} \mathrm{CO}$ ), 7.59 (s, 8H, ArH), 8.21 (br. t, $\left.4 \mathrm{H}, \mathrm{NHCH}_{2} \mathrm{CH}_{2} \mathrm{~N}^{+}\right), 8.51\left(\mathrm{t},{ }^{3} \mathrm{~J}_{\mathrm{HH}}=5.7 \mathrm{~Hz}, 4 \mathrm{H}, \mathrm{CONH}\right), 8.90$ 
$\left(\mathrm{t},{ }^{3} \mathrm{~J}_{\mathrm{HH}}=5.4 \mathrm{~Hz}, 4 \mathrm{H}, \mathrm{CONH}\right) .{ }^{13} \mathrm{C}$ NMR $(100 \mathrm{MHz}, 298 \mathrm{~K}$, DMSO$\left.\mathrm{d}_{6}\right) \delta: 170.14,168.84,168.43,163.49,157.59,146.49,131.42$, 127.97, 119.60 (q), 72.17, 60.98, 57.08, 56.37, 55.59, 41.92, 41.13, 34.35, 32.47, 31.21, 14.47, 7.90. El. anal. calcd for $\mathrm{C}_{112} \mathrm{H}_{164} \mathrm{~F}_{24} \mathrm{~N}_{20} \mathrm{O}_{40} \mathrm{~S}_{12}$ : C 41.12\%, H 5.05\%, N 8.56\%, S 11.76\%. Found: C $41.07 \%$, H 5.15\%, N 8.49\%, S 11.92\%. MS (ESI): calcd for $\left[\mathrm{M}-3 \mathrm{~N}^{-}\left(\mathrm{SO}_{2} \mathrm{CF}_{3}\right)_{2}\right]^{3+} m / z=810.3,\left[\mathrm{M}-4 \mathrm{~N}^{-}\left(\mathrm{SO}_{2} \mathrm{CF}_{3}\right)_{2}\right]^{4+} \mathrm{m} /$ $z=537.6$, found $\mathrm{m} / z=810.0,537.7$. IR $\nu_{\text {max }}$ : 1181 (COC), 1666 $(\mathrm{C}=\mathrm{O}), 2968,3363(\mathrm{~N}-\mathrm{H})$.

$5,11,17,23$-Tetra-tert-butyl-25,26,27,28-tetrakis $\left[N\right.$ - $\left(2^{\prime}, 2^{\prime}\right.$-diethyl$2^{\prime}-\{($ ethoxycarbonyl $[S$-methyl $]$ methyl)amidocarbonylmethyl $\}$ ammoniumethyl)carbomoylmethoxy]-2,8,14,20-tetrathiacalix[4] arene tetra[bis(trifluoromethylsulfonyl)imide] (1,3-alternate 72). Yield: $0.13 \mathrm{~g}$ (96\%), mp $62{ }^{\circ} \mathrm{C} .{ }^{1} \mathrm{H}$ NMR (400 MHz, $298 \mathrm{~K}$, DMSO- $\left._{6}\right) \delta: 1.19\left(\mathrm{t},{ }^{3} \mathrm{~J}_{\mathrm{HH}}=7.1 \mathrm{~Hz}, 12 \mathrm{H}, \mathrm{CH}_{3} \mathrm{CH}_{2} \mathrm{O}-\right), 1.21(\mathrm{~s}$, $\left.36 \mathrm{H},\left(\mathrm{CH}_{3}\right)_{3} \mathrm{C}\right), 1.32\left(\mathrm{~d},{ }^{3} J_{\mathrm{HH}}=7.3 \mathrm{~Hz}, 12 \mathrm{H}, \mathrm{CH}_{3} \mathrm{CH}\right), 1.34\left(\mathrm{t},{ }^{3} J_{\mathrm{HH}}\right.$ $\left.=7.2 \mathrm{~Hz}, 24 \mathrm{H}, \mathrm{CH}_{3} \mathrm{CH}_{2} \mathrm{~N}^{+}\right), 3.49\left(\mathrm{~m}, 8 \mathrm{H}, \mathrm{NHCH}_{2} \mathrm{CH}_{2} \mathrm{~N}^{+}\right), 3.51-$ $3.58\left(\mathrm{~m}, 24 \mathrm{H}, \quad \mathrm{N}^{+} \mathrm{CH}_{2} \mathrm{CH}_{3}, \quad \mathrm{NHCH}_{2} \mathrm{CH}_{2} \mathrm{~N}^{+}\right), \quad 4.07(\mathrm{~s}, 8 \mathrm{H}$, $\mathrm{OCH}_{2} \mathrm{CO}$ ), 4.09-4.11 (m, 16H, N $\left.{ }^{+} \mathrm{CH}_{2} \mathrm{CO}, \mathrm{CH}_{3} \mathrm{CH}_{2} \mathrm{O}-\right)$, $4.30(\mathrm{~m}$, $\left.4 \mathrm{H}, \mathrm{CH}_{3} \mathrm{CH}\right), 7.60(\mathrm{~s}, 8 \mathrm{H}, \mathrm{ArH}), 8.23\left(\mathrm{t},{ }^{3} \mathrm{~J}_{\mathrm{HH}}=6.7 \mathrm{~Hz}, 4 \mathrm{H}\right.$, $\mathrm{NHCH}_{2} \mathrm{CH}_{2} \mathrm{~N}^{+}$), 9.11 (d, $\left.{ }^{3} J_{\mathrm{HH}}=6.7 \mathrm{~Hz}, 4 \mathrm{H}, \mathrm{CONH}\right) .{ }^{13} \mathrm{C} \mathrm{NMR}$ (100 MHz, 298 K, DMSO-d ${ }_{6}$ ) $\delta:$ 171.63, 167.99, 162.83, 156.91, 146.01, 133.00, 127.43, 119.48 (q), 70.54, 60.85, 56.36, 55.93, 55.17, 47.92, 33.84, 31.96, 30.83, 16.56, 13.97, 7.34. El. anal. calcd for $\mathrm{C}_{108} \mathrm{H}_{160} \mathrm{~F}_{24} \mathrm{~N}_{16} \mathrm{O}_{36} \mathrm{~S}_{12}$ : C 41.85\%, H 5.20\%, N 7.23\%, S $12.42 \%$. Found: C $42.08 \%$, H 5.38\%, N 6.97\%, S $12.09 \%$. MS (ESI): calcd for $\left[\mathrm{M}-4 \mathrm{~N}^{-}\left(\mathrm{SO}_{2} \mathrm{CF}_{3}\right)_{2}\right]^{4+} \mathrm{m} / z=494.6$, found $m / z=$ 494.6. IR $\nu_{\max }: 1181$ (COC), $1680(\mathrm{C}=\mathrm{O}), 2969,3361(\mathrm{~N}-\mathrm{H})$.

\section{Acknowledgements}

The financial support of RFBR (16-33-60141 mol_a_dk) and the Program of the President of the Russian Federation for the State support of young Russian scientists-scholarships (CP3597.2016.4) is gratefully acknowledged.

\section{Notes and references}

1 K. Shimojo and M. Goto, Chem. Lett., 2004, 33, 320-321.

2 K. Shimojo and M. Goto, Anal. Chem., 2004, 76, 5039-5044.

3 N. Sieffert and G. Wipff, J. Phys. Chem. A, 2006, 110, 11061117.

4 N. Sieffert and G. Wipff, J. Phys. Chem. B, 2006, 110, 1949719506.

5 T. Sun, Z. Wang and X. Shen, Inorg. Chim. Acta, 2012, 390, 8-11. 6 P. K. Mohapatra, P. Kandwal, M. Iqbal, J. Huskens, M. S. Murali and W. Verboom, Dalton Trans., 2013, 42, 4343-4347.

7 F. Yang, F. Guo, Z. Jiao, C. Li and J. Ye, J. Iran. Chem. Soc., 2012, 9, 327-332.

8 B. Aitken, M. Lee, M. Hunley, H. Gibson and K. Wagener, Macromolecules, 2010, 43, 1699-1701.

9 J. Weng, C. Wang, H. Li and Y. Wang, Green Chem., 2006, 8, 96-99.

10 J. Pernak, M. Smiglak, S. T. Griffin, W. L. Hough, T. B. Wilson, A. Pernak, J. Zabielska-Matejuk, A. Fojutowski, K. Kita and R. D. Rogers, Green Chem., 2006, 8, 798-806.
11 X. L. Yuan, S. J. Zhang and X. M. Lu, J. Chem. Eng. Data, 2007, 52, 596-599.

12 S. Werner, M. Haumann and P. Wasserscheid, Annu. Rev. Chem. Biomol. Eng., 2010, 1, 203-230.

13 Y. A. Gao, Z. H. Li, J. M. Du, B. X. Han, G. Z. Li, W. G. Hou, D. Shen, L. Q. Zheng and G. Y. Zhang, Chem.-Eur. J., 2005, 11, 5875-5880.

14 P. Montes-Navajas, A. Corma and H. J. Garcia, J. Mol. Catal. A: Chem., 2008, 279, 165-169.

15 N. Inazumi, S. Yamamoto and Y. Sueishi, J. Inclusion Phenom. Macrocyclic Chem., 2007, 59, 33-39.

16 D. N. Shurpik, L. S. Yakimova, I. K. Rizvanov, V. V. Plemenkov and I. I. Stoikov, Macroheterocycles, 2015, 8, 128-134.

17 L. S. Yakimova, D. N. Shurpik, L. H. Gilmanova, A. R. Makhmutova, A. Rakhimbekova and I. I. Stoikov, Org. Biomol. Chem., 2016, 14, 4233-4238.

18 R. V. Nosov and I. I. Stoikov, Macroheterocycles, 2014, 7, 345350.

19 I. Spendlikova, J. John, V. Cuba, J. Jirasek and P. Lhotak, J. Radioanal. Nucl. Chem., 2015, 304, 257-262.

20 M. MacKova, M. Himl, J. Budka, M. Pojarova, I. Cisarova, V. Eigner, P. Curinova, H. Dvorakova and P. Lhotak, Tetrahedron, 2013, 69, 1397-1402.

21 D.-D. Zheng, D.-Y. Fu, Y. Wu, Y.-L. Sun, L.-L. Tan, T. Zhou, S.-Q. Ma, X. Zha and Y.-W. Yang, Chem. Commun., 2014, 50, 3201-3203.

22 Y. Zhou, H. Li and Y.-W. Yang, Chin. Chem. Lett., 2015, 26, 825-828.

23 M. Yamada, Y. Ootashiro, Y. Kondo and F. Hamada, Tetrahedron Lett., 2013, 54, 1510-1514.

24 M. Yamada, M. Rajiv Gandhi, U. M. R. Kunda and F. Hamada, J. Inclusion Phenom. Macrocyclic Chem., 2016, 85, 1-18.

25 A. D. Iampolska, S. G. Kharchenko, Z. V. Voitenko, S. V. Shishkina, A. B. Ryabitskii and V. I. Kalchenko, Phosphorus Sulfur Relat. Elem., 2016, 191, 174-179.

26 T. Ogoshi, N. Ueshima, T. Yamagishi, Y. Toyota and N. Matsumi, Chem. Commun., 2012, 48, 3536-3538.

27 A. Kokorin, Ionic liquids: theory, properties, new approaches, InTech, 2011.

28 F. Hurley and T. Wler, J. Electrochem. Soc., 1951, 98, 203206.

29 J. Hallet and T. Welton, Chem. Rev., 2011, 111, 3508-3576.

30 P. Wasserscheid and T. Welton, Ionic liquids in synthesis: second edition, John Wiley \& Sons, Inc., 2009.

31 S. L. Craig, Angew. Chem., Int. Ed., 2009, 48, 2645-2647.

32 J. Lopes and A. Padua, J. Phys. Chem. B, 2006, 110, 33303335.

33 J. H. Davis, K. J. Forrester and T. Merrigan, Tetrahedron Lett., 1988, 39, 8955-8958.

34 K. Singh, D. G. Marangoni, J. G. Quinn and R. D. Singer, J. Colloid Interface Sci., 2009, 335, 105-111.

35 E. A. Andreyko, P. L. Padnya and I. I. Stoikov, J. Phys. Org. Chem., 2015, 28, 527-535.

36 Y. Huo, S. Xia, Y. Zhang and M. Peisheng, Ind. Eng. Chem. Res., 2009, 48, 2212-2217. 
37 M. K. Rauf, R. Mushtaq, A. Badshah, R. Kingsford-Adaboh, J. J. E. K. Harrison and H. Ishida, J. Chem. Crystallogr., 2013, 43, 144-150.

38 P. L. Padnya, E. A. Andreyko, O. A. Mostovaya, I. Kh. Rizvanov and I. I. Stoikov, Org. Biomol. Chem., 2015, 13, 5894-5904.

39 S. Zhang, N. Sun, X. He, X. Lu and X. Zhang, J. Phys. Chem. Ref. Data, 2006, 35, 1475-1517.
40 E. A. Andreyko, P. L. Padnya, R. R. Daminova and I. I. Stoikov, $R S C A d v$., 2014, 4, 3556-3565.

41 E. A. Andreyko, P. L. Padnya and I. I. Stoikov, Colloids Surf., A, 2014, 454, 74-83.

42 F. Gutmann and L. E. Lyons, Organic Semiconductors, Wiley, New York, 1967. 